\title{
Development of Synthetic Coal Char Simulant for Microwave Conversion Studies: A Computationally-Driven Approach
}

\author{
Kevin A. Hager \\ West Virginia University, kah0068@mix.wvu.edu
}

Follow this and additional works at: https://researchrepository.wvu.edu/etd

Part of the Computer-Aided Engineering and Design Commons, Engineering Physics Commons, Materials Chemistry Commons, Numerical Analysis and Computation Commons, Other Materials Science and Engineering Commons, and the Other Mechanical Engineering Commons

\section{Recommended Citation}

Hager, Kevin A., "Development of Synthetic Coal Char Simulant for Microwave Conversion Studies: A Computationally-Driven Approach" (2021). Graduate Theses, Dissertations, and Problem Reports. 10203. https://researchrepository.wvu.edu/etd/10203

This Thesis is protected by copyright and/or related rights. It has been brought to you by the The Research Repository @ WVU with permission from the rights-holder(s). You are free to use this Thesis in any way that is permitted by the copyright and related rights legislation that applies to your use. For other uses you must obtain permission from the rights-holder(s) directly, unless additional rights are indicated by a Creative Commons license in the record and/ or on the work itself. This Thesis has been accepted for inclusion in WVU Graduate Theses, Dissertations, and Problem Reports collection by an authorized administrator of The Research Repository @ WVU. For more information, please contact researchrepository@mail.wvu.edu. 


\title{
Development of Synthetic Coal Char Simulant for Microwave Conversion Studies: A Computationally-Driven Approach
}

\author{
Kevin Andrew Hager \\ Thesis submitted to the \\ Benjamin M. Statler College of \\ Engineering and Mineral Resources \\ at West Virginia University \\ in partial fulfillment of the requirements \\ for the degree of \\ Master of Science \\ in \\ Mechanical Engineering \\ Terence Musho, Ph.D., Chair \\ Edward Sabolsky, Ph.D. \\ Christina Wildfire, Ph.D. \\ Department of Mechanical and Aerospace Engineering \\ Morgantown, West Virginia \\ 2021
}

Keywords: Powder River Basin coal char, PRB coal char, CT image, dielectric, Nicolson-Ross-Weir, synthetic PRB coal char, finite element analysis, discrete element modelling, conductivity, simulation

Copyright 2021 Kevin Hager 


\section{ABSTRACT \\ Development of Synthetic Coal Char Simulant for Microwave Conversion Studies: A Computationally-Driven Approach}

Kevin Andrew Hager

Recent experimental demonstration of new reaction windows for coal char/methane reactions that are less energy-intensive, provides innovation for modular reactors. However, the correlation of the exact mechanism for the enhancement of these reaction windows is not certain. This study investigates the simplification of these experimental studies by developing a well-characterized coal char simulant. The approach involves using a computational approach to screen macroscopic composition to replicate the dielectric and compositional response of actual char. This study is focused on PRB coal char. A discrete element method (DEM) technique was used to simulate the packing of coal chars to give the precise distribution of particle sizes. Micro-CT images of actual coal char were taken and the Feret diameter and particle count were used in DEM simulation. Using the packed DEM geometry, a finite element analysis (FEA) using COMSOL was utilized to solve Maxwell's equations to match the experimental dielectric properties. Once the required volume fraction and constituents were known, the coal char simulate was synthesized. The comparison of simulation dielectric and actual char dielectric was within $5 \%$ error. The synthetic char was experimentally synthesized and the density of the synthetic dielectric was determined to be $0.5 \mathrm{~g} / \mathrm{cc}$ and the actual char had a density of $0.4 \mathrm{~g} / \mathrm{cc}$. It was determined that the imaginary part of the synthetic char was much larger than the actual char. This was reasoned to be due to the larger electrical conductivity associated with the synthetic char material. Further investigation of the actual char through both optical and scanning tunneling microscopy revealed significant amount of ash content surrounding the char. It is hypothesized that this ash layer coating the char as a result of pyrolysis process is leading to decreased electrical conductivity. A similar FEA approach was used to investigate the particle morphology of a magnetite $\left(\mathrm{Fe}_{3} \mathrm{O}_{4}\right)$ catalyst embedding on a coal char substrate to understand the localized temperature and electric field enhancements. It was determined that a particle shape significantly influences electric field and localized temperatures. In the absence of the shaped particle the peak electric field strength and subsequently, the volumetric heat flux was two orders of magnitude lower. An optimal geometry and volume fraction to enhance these localized field effects were found during the study. 


\section{ACKNOWLEDGMENTS}

Dr. Terence Musho, for the copious amount of advice and patience needed to advise me in my studies/professional development. From whom I have inherited a great multitude of information in a short amount of time and enabled me to become a better student and professional during the short time as my mentor.

Dr. Edward Sabolsky, for the expertise in fabricating - and implementing, the experimental portion of the study. His advice on percolation theory and theory on the chemical engineering side of the study was incalculably valuable.

I would be remiss if I didn't mention Anthony Carter (Dr. Sabolsky's graduate Master's student) for the collaboration and implementation of the many changes requested during the experimental portion. His steadfast approach to many hours laboring in the lab to bring the simulate into reality was much appreciated.

Dr. Christina Wildfire for her work with VNA at NETL and advice on elucidating dielectric properties of the coal char using the microwave reactor and data analysis.

Dr. Wildfire's fellow researcher, Candice, also deserves my thanks for facilitating said analysis.

Robert Tempke for helping to teach me the basics of image analysis and cluster computing and aid with succeeding in graduate school in general.

The late Dr. William E. Dieterle, experimental particle physicist from Berkeley who had more faith in me than I had deserved. He will always be in my memory in my academic and professional career.

Last but not least, my parents, Keith and Carla Hager, whose patience and understanding helped make me a better person. Thank you for everything.

This work was supported by NETL and DE-FE0026825 - University Coalition for Fossil Energy Research 


\section{TABLE OF CONTENTS}

Page

ACKNOWLEDGMENTS. . . . . . . . . . . . . . . . . iii

\section{Chapter}

1. INTRODUCTION . . . . . . . . . . . . . . . . . . . 1

Rationale.................................. 1

Powder River Basin $(\mathrm{PRB})$ Coal $\ldots \ldots \ldots \ldots \ldots \ldots \ldots \ldots \ldots \ldots$

Computed Tomography $\ldots \ldots \ldots \ldots \ldots \ldots \ldots \ldots \ldots \ldots \ldots \ldots$

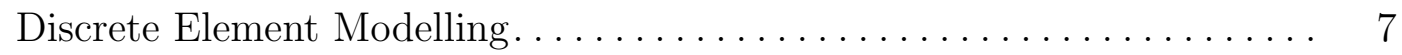

Finite Element Analysis . . . . . . . . . . . . . . . . . . . 9

2. RESULT AND DISCUSSION . . . . . . . . . . . . . . . 12

Methods of Materials:. . . . . . . . . . . . . . . . . . . . . . . . 12

Preparation of Computational Analysis of PRB Coal Char. . . . . . . . . . 12

ImageJ and Paraview Analysis $\ldots \ldots \ldots \ldots \ldots \ldots \ldots \ldots \ldots \ldots \ldots \ldots \ldots$

Discrete Element Modeling . . . . . . . . . . . . . . . . . . . 14

Volume Fraction Analysis of PRB Coal Char. . . . . . . . . . . . . . 16

COMSOL Simulation $\ldots \ldots \ldots \ldots \ldots \ldots \ldots \ldots \ldots \ldots \ldots \ldots \ldots \ldots \ldots$

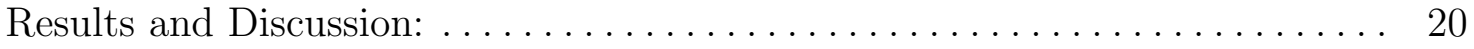

Density of PRB Coal Char and Synthetic Char................. 20

Verification of COMSOL-simulated PRB Coal Char. .............. 21 
Examination of Fitting Scattering Parameters Using a Modified Ap-

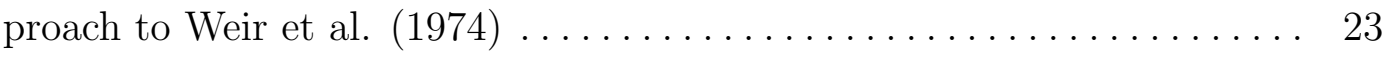

Modification and Analysis of Conductivity of sp2-hybridized Carbon

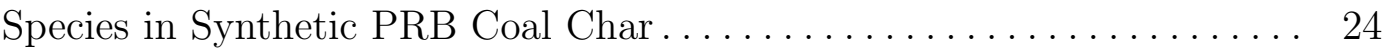

Optical Analysis of PRB coal char versus Synthetic Char . . . . . . . . . 27

Further Modification of Synthetic Coal Char to Increase Fidelity of Ac-

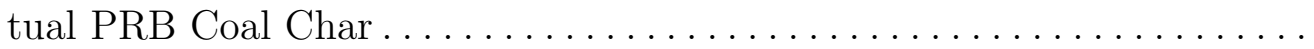

Implementation and Experimental Verification of Dielectric Properties of $\mathrm{Fe}_{3} \mathrm{O}_{4}$ Nanoflakes in Micro-Reactor Using COMSOL . . . . . . . . . . . . 31 Simulation of $\mathrm{Fe}_{3} \mathrm{O}_{4}$ Nanoflakes in Micro-Reactor on Synthetic Coal Char

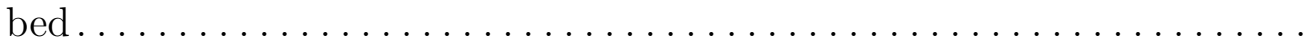

Analysis of Compression on the Electrical Conductivity of Synthetic Coal Char.

Analysis of Electrical Conductivity of Pyrolyzed PRB Coal . . . . . . 37

Simulation Results involving Thermal Power Generation of Shaped $\mathrm{Fe}_{3} \mathrm{O}_{4}$

Microparticles on Bed of Synthetic Char.

3. CONCLUSION. . . . . . . . . . . . . . . . . . . . 57

BIBLIOGRAPHY . . . . . . . . . . . . . . . . . . . . . . . 59

Appendices . . . . . . . . . . . . . . . . . . . . . . . . . . . . . . 63

RNG Code for Particle Placement in COMSOL model . . . . . . . . . . . 63

NRW Polynomial Interpolation Code . . . . . . . . . . . . . . . . . 65 


\section{LIST OF FIGURES}

Figure 2.1 Volume reconstructed data of PRB Coal taken using micro-CT machine. Subfigure A illustrates the selection of particles edges using ImageJ. Subfigure B is a slice of the raw reconstructed CT image in ImageJ. The diameter of the circular image is approximately 1108 microns in diameter. . . . . . . . . . . . . . . . . . . . . . . . . . . .

Figure 2.2 Subfigure A is histogram of the particle diameter (Feret diameter) of the particles taken CT images of PRB char. It is noted that the distribution is skewed. Subfigure B is an image the DEM simulation of particle filling and the instantaneous histogram of particle diameter. Note the similar skewed distribution between A and B............ 14

Figure 2.3 Illustration of a DEM-simulation of char where the particles are approximated as spheres. A coaxial test cell is being filled and compressed in the simulation. The test cell has an outer diameter of 7 $\mathrm{mm}$ and inner diameter of $3 \mathrm{~mm}$. The particle diameters are based on the distribution of particles sizes from the micro CT images..........

Figure 2.4 Images from COMSOL RF simulation. Subfigure A is a physics-based mesh for the electromagnetic wave study. Subfigure B is a selection (highlighted in blue) of individual particles in the simulation based on RNG macro. Subfigure C is top view of packed of the spheres with varying radii. Subfigure D is a multi-slice with electric field color contours. Note the enhanced electric field between particles that highly influence dielectric response in granular dielectric materials.......... 18 
Figure 2.5 Calculated COMSOL permittivity values using the NRW method as a function of frequency from 1 to $10 \mathrm{GHz}$. These values are the synthetic char material outlined in Table $2.2 \ldots \ldots \ldots \ldots \ldots \ldots \ldots \ldots \ldots \ldots \ldots$

Figure 2.6 Polynomial fit of real (A) and imaginary (B) components of S11 calculated in COMSOL for synthetic char material. Subfigure C and D are the corresponding transmission magnitude and angle. Note the

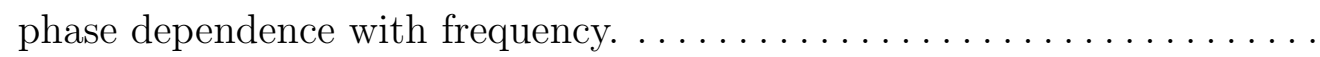

Figure 2.7 Illustration of the voltage distribution in an electrostatic simulation of synthetic char. The bottom boundary has a voltage of $1 \mathrm{~V}$ and the top is grounded. Notice that the conductive particles closest to the voltage boundary conduct the voltage until they interface with a less conductive particles. The overall current will depend on the network of conductive particles within domain..................

Figure 2.8 Optical micrographs of synthetic coal char (A-B) and actual PRB coal char (C-D). The black material in all the micrographs is carbon. The white material is ash and the red particles are hematite. Note the red particle in Subfigure C for the actual char. Moreover, it was noted that the ash content collects on the surface for actual char $(\mathrm{C}-\mathrm{D}) \ldots \ldots \ldots \ldots$

Figure 2.9 X-ray diffraction spectra of the Powder River Basin coal char (red) and Synthetic Powder River Basin coal char (green). The peak centered around $27-28^{\circ}$ illustrates the strong presence of pyrolyzed graphitic

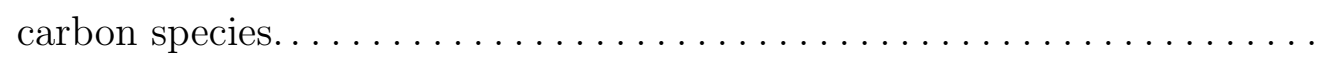


Figure 2.10 Scanning electron microscopy of the second version of Synthetic Powder River Basin coal char synthesized with the addition of more graphite species and increased homogeneity of ash coating using spin-drying methods as opposed to simple mechanically-mixing the original PRB simulate formula with $15 \%$ graphite species. . . . . . . . . . .

Figure 2.11 Scattering parameter results of COMSOL simulation of a mono-flake of magnetite $\left(\mathrm{Fe}_{3} \mathrm{O}_{4}\right)$ suspended in Nitrogen gas $\left(\mathrm{N}_{2}\right)$. It is important to note that S11 is equal to the total reflective power because S21 (transmission) is zero. The return losses match what Yang and Zhao et al. have tested previously in their respective groups. . . . . . . . . 33

Figure 2.12 Scatter plot of magnetite nanodisc (flake) versus thickness in microns at the target frequency $2.50 \mathrm{GHz}$ created from a parameter sweep in COMSOL. The optimal thickness appears to be at 1.5 micron thickness

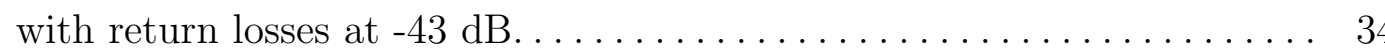

Figure 2.13 SEM imaging of $\mathrm{Fe}_{2} \mathrm{O}_{3}$ (subfigure A) and $\mathrm{Fe}_{3} \mathrm{O}_{4}$ (subfigure C) micro-platelet formations from literature (Zhao et al. 2018) [1] vs. Samples of $\mathrm{Fe}_{2} \mathrm{O}_{3}$ (subfigure B) and $\mathrm{Fe}_{3} \mathrm{O}_{4}$ (subfigure D) micro-platelet formation performed by the experimental portion of the group . . . . . . .

Figure 2.14 Images of Synthetic Coal Char (version 3) - spin-coated in ash to match XRD of the actual PRB coal char. Note the increased inclusion growth of fine ash matter (protuberances) on the surface of the synthetic coal particle in both subfigures, perfectly mimicking the actual PRB coal char images. . . . . . . . . . . . . . . . 
Figure 2.15 Current-voltage chart (also known as IV-curve) of the various trials of the synthetic PRB (version 2) with different pyrolyzed temperatures. Note the further decrease in conductivity in synthetic PRB (version 3). . 38

Figure 2.16 Current-voltage chart of pyrolyzed PRB coal char at temperatures at or beyond $900^{\circ} \mathrm{C}$. This resulted in a far greater conductivity than previous torrification at $500^{\circ} \mathrm{C}$, thereby verifying Yin et al..........

Figure 2.17 Helpful diagram illustrating the direction of the electric, and by extension, magnetic field across the reactor domain. Subfigure A is where the simulation sets the electric field at 0 degrees with respect to (w.r.t) the particle/reactor. Subfigure B is where the simulation aligns the electric field at 85 degrees normal to the plane of the reactor where the particles and syn char bed rest...................... 41

Figure 2.18 Comparison of thickness of square magnetite particles with power generation with a bed of syn char at $10 \mathrm{~S} / \mathrm{m}$. The changing of thickness negligibly affects power generation. .................... 42

Figure 2.19 Comparison of electrical conductivity of square magnetite particles with power generation with a bed of syn char at $495 \mathrm{~S} / \mathrm{m}$ (syn char version 3 in subfigure A) and $10 \mathrm{~S} / \mathrm{m}$ (inert, pyrolzyed PRB coal char in

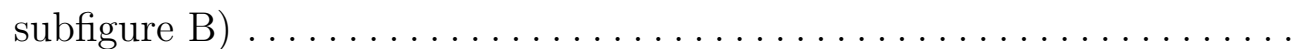

Figure 2.20 Comparison of power generation of just the bed of syn char (subfigure A) and the presence of square magnetite particles (subfigure B). The presence of particles multiplies the power generated by several fold..... 44 
Figure 2.21 Comparison of power generation of just the bed of syn char (subfigure A) and the presence of square magnetite particles (subfigure B). Clearly depicted is the 3-4 magnitude increase in power density output. . . . . . .

Figure 2.22 Comparison of the electric field norm $(\mathrm{V} / \mathrm{m})$ of triangular (subfigure $\mathrm{A}$ ) and square particles (subfigure B). Similar to the power generation plots, the electric field for quadrilateral particles exceeds the triangular particles by several magnitudes. . . . . . . . . . . . . . . . 46

Figure 2.23 Comparison of the electric field norm $(\mathrm{V} / \mathrm{m})$ of triangular (subfigure A) and square particles (subfigure B). Electric field is toward the viewer in the xy-plane. Notice the electric field vectors and their interactions of the two shaped particles, showing the influence of shaped-particles on incident microwave radiation.

Figure 2.24 The norm of the Poynting vector that details how the energy of the electromagnetic waves propagate through media. Triangular particles (Subfigure A), shows that the Poynting vector norm pervades throughout the triangle particle with little difference of flux except for the periphery of the shape. Contrasted to that of the square particle (subfigure B), which shows the focusing of the electromagnetic energy away from the edges of the square prism................ 48

Figure 2.25 Isosurface/multislice representation of the magnetic flux density (B-field) with magnetic flux field vectors illustrating the edge effects of the triangular particle. The isosurface coupled with the arrow depiction of B-field vectors shows how the magnetic field lines concentrate in the triangular-shaped particle........................... 49 
Figure 2.26 Volumetric representation of the magnetic flux density (B-field) of a square-shaped particle. Red arrows indicate direction of B-field. The magnetic flux density clearly depicts the poles of the square prism. . . . . 51

Figure 2.27 Compilation of contour plots illustrating the several magnitude order difference in power loss due to magnetic loss (subfigure A) and electrical (subfigure B) loss. Subfigure C is the total loss, which is dominated by the electrical field contribution, in accordance with Maxwell's Equations. 52

Figure 2.28 Constant area fraction comparison between the triangle (subfigure A) and square-shaped particles (subfigure B). When the characteristic length parameters were swept across in the FEA simulation, the power generation was several fold larger in square-shaped particles than triangular-shaped entities. . . . . . . . . . . . . . . 53

Figure 2.29 Visual depiction of the energy dispersion of the shaped particles. The focusing of electric field energy at the tips of the triangles (subfigure A) can clearly be seen. The dissipation of energy for square-shaped particles (subfigure B) seems to be peripheral all along the edges of the magnetite particle........................ 54

Figure 2.30 Snapshot in time of the heating effect of the magnetite particle when irradiated with $2000 \mathrm{~W}$ microwave energy (non-pulsed) incident 85 degrees to the plane of the particle and bed. Subfigure A (triangle) shows the resultant heating at 0.06 seconds. Subfigure B (square) shows the resultant heating at 0.1 seconds. In both scenarios, the temperature of the local particle surroundings increases dramatically. . . $\quad 55$ 


\section{LIST OF TABLES}

Table 2.1 Comparison of experimental volume fraction analysis and DEM results for filling- and after-compression. The left table is the experimental volume fraction inferred from the CT images at $44.82 \%$. The right table refers to the DEM simulation in Yade with $41.85 \%$ volume fraction when filled (Fill) and 58.93\% volume fraction when compressed (Ram).. 16

Table 2.2 Initial synthetic char volume fraction that reproduce the dielectric response of actual PRB char. These volume fractions were determined using a combination of COMSOL simulations, Raman spectroscopic analysis of graphitic carbon bands, and visual determination based on stereological techniques...............................

Table 2.3 DC electrical conductivity values calculated from COMSOL-derived IV curves. Present are outlier values; it can be inferred that particle placement supplanted graphite load for contribution towards conductivity. Conductivity depends on conductive network of particles so several values were conducted and averaged. Conductivity plateaus

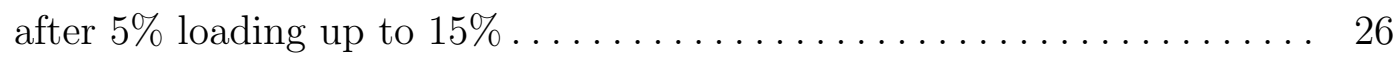




\section{CHAPTER 1}

\section{INTRODUCTION}

\section{Rationale}

The primary motivation of this paper is to determine the fundamental mechanisms and advantages of microwave-based conversion of coal. Our approach is to simplify the representation of coal and to maintain consistency of experiments to permit fundamental scientific understanding using ab initio principles to model novel, modularized, micro-reactors utilizing the non-trivial interaction with low-ranking coal. The coal specimen of interest is the Powder River Basin coal (PRB) sourced from the Black Thunder mines in the basins of Wyoming and was chosen primarily for its relatively high carbon content with its low sulfur and moderate moisture content - viable for a more environmentally friendly application of these modular reactors with pyrolysis processes. These complex electromagnetic interactions with the pyrolyzed PRB coal can provide a robust framework from which to aid catalysis of reduction reactions of aliphatic moieties embedded within the coal matrix with magnetic nanoparticles providing results in the form of micro-antennae arrays to aid in catalyzing the

processing of hydrocarbons in a less energy-intensive way. [2] Extensive literature [3, 4, 5] has been performed on using carbonaceous powders to absorb microwave radiation as carbonaceous materials are known to be efficient absorbers of microwave bands and could be main heating sources for carbo-thermic reductions of catalytic processes such as using transition metal oxides via microwave induction. Coal specimens are highly localized, to the degree that a representative coal sample cannot be trivially obtained by visual inspection and chemical analysis alone. The complex interchange between extant macromolecular groups and preternatural crystalline alignment leads to large deviances into any sort of generalized characteristic models, but certain trends do occur in the creation of the coal char sample that 
can be reverse-engineered into a pseudo-coal char sample called synthetic coal char or "syn char". This model should be built using ab initio principles and from basic components of the coal sample. Average structural properties of coal include interspersed lithotype regions of organic hydrocarbons that form the backbone of the coal structure, with varying sizes from centimeters in scale with porous mineral nodules on the order of millimeters on lithotype boundaries. [6] More minute details include the microscopic organic material left over from vegetation decomposition in the form of structures called "macerals" which are microscopic to nanoscopic in range of size. Variable carbon content, mixed with smaller amount of volatile organic compounds and moisture, tends to be the basic molecular building blocks of coal specimens. Coal char, however, is devoid of these organic moieties and moisture post-pyrolysis, such that the only residual molecular content is the carbonaceous material with interspersed ash content such as silicates, potash, rare earth metals, iron oxides, and molten salts throughout the charred sample. This process can be at temperatures around $500^{\circ} \mathrm{C}$ (torrification) and much higher at temperatures exceeding $900^{\circ} \mathrm{C}$ (what this study refers to as "pyrolyzed"). Thanks to the infancy of polymer science in the mid-to-late twentieth century, the understanding of the macromolecular and structural building blocks of coal was updated to describe coal as a cross-linked structure of individual monomers of aromatic and non-aromatic hydrocarbons with a matrix embedded by inorganic mineral content. Zhihong [7] states that coal is the resultant mixture of macromolecular components, medium molecular components (aliphatic and aromatic compounds) and small molecular components (heteroatomous-bridging of nanoparticles and with Nitrogen and Oxygen linkages). Using these presuppositions, it became more obvious as to a procedure to create a realistic facsimile of coal and its components in a laboratory setting. [8] In order to properly model the PRB coal char, it was determined to be beneficial to use discrete-element modeling (DEM) to create fully virtualized reactors to simulate the highly inhomogeneous structure of a sample of PRB coal char. Once the model is constructed via a Computer-aided design (CAD) interface (and using previously obtained information of the PRB coal's morphology/chemical 
composition), the usage of finite-element analysis techniques (FEA) can be implemented. FEA is commonly used to solve computationally expensive partial differential equations of high order and non-linearity by partitioning solutions via approximation methods at the interfaces of the constructed mesh and solving boundary-value problems. [9] The resultant simulation of a virtual reactor and synthetic coal char provides a known control of inputs that approximate the Powder River Basin coal and can be verified in conjunction with common characterization methods, thus leading to a better survey of the dielectric responses of the pyrolyzed coal char and the underlying chemistry enhancement. The purpose of the pyrolysis of coal is many-fold. Devolatilization and desulfurization of organic compounds are energy-intensive processes that need to physically and chemically occur in order to provide useful hydrocarbon product formation. Thus, microwave energy is an unconventional heating source of coal by utilizing the dielectric response of absorbing the electromagnetic waves and creating localized regions of high heat, heating the coal from the inside-out by rapid, non-contact polarization of the coal's electric and magnetic fields governed by the sample's permittivity and permeability of which the coal's composition may vary strongly; i.e. the larger these values are, the more the incident EM-radiation is absorbed and transferred into thermal energy. [10] It has been shown that localized regions of plasma can form from the sharp points and edges of granular coal, leading to the formation of carboxyl radicals that can then be processed into syngas and other polymerization processes. [11] The electrochemical properties of coal char (specifically the dielectric properties) can be derived from vectornetwork analysis (VNA), where a coaxial airline is packed with the sample and the resultant scattering matrix (S-parameters) can then be utilized using the Nicolson-Ross-Weir method (NRW) to extract the complex electric permittivity and magnetic permeability from the S-parameters, in this particular case using the standard 2-port approach. [12] This method is a reliable and accurate way to measure the dielectric properties of specimens, as well as the gain and lossiness of the sample. To effortlessly probe the electrochemical properties of the coal char and to create a very efficient method of performing VNA studies without 
copious amounts of time and money in the physical lab, this paper focuses on its relatively novel usage of performing the exact metric in a purely virtual setting, such as the COMSOL FEA software, to scale up trials and simulations that a wet lab could not hope to perform on an efficient economical/temporal basis. The praxis of the COMSOL software then comes into its own with the ability to add multi-physics packages to the DEM model created in a CAD-neutral setting, and solving Maxwell's equations under various conditions set by the

user. This can be automated using machine learning techniques or through the usage of built-in macros to provide seamless transitions into other modes of characterization. The electromagnetic waves physics module and electrostatics modules were used extensively up to this point in this work, but further analysis using the thermal physics package included in COMSOL was utilized to probe the non-linear thermal effects of the microwave pyrolysis of the char simulate and is covered towards the end of the study. It is hypothesized that microwave-induced losses can be optimized by utilizing a carbon-rich material such as coal char and that the coal char simulate's morphology and admixture can further augment the dielectric loss to a degree that can be suited for more effective micro-scale reactors.

\section{Powder River Basin (PRB) Coal}

\section{Background Information}

The core sample of this study involves the Powder River Basin (PRB) coal char, coming from the eponymous Powder River Basin located in northeastern regions of Wyoming and southeastern Montana. PRB coal's heating throughput is classified as roughly 8,500 BTU/lb, which makes it less energy-dense as Appalachian coal which averages 10,000 BTU/lb. [13] This made Powder River Basin coal a substandard coal for energy-use until the advent of clean air proposals of the 1980's and 1990's attempted to reverse and eliminate the unfortunate side-effect of coal combustion, the production of $\mathrm{SO}_{2}$. The prevention of acid rain led to increased interest in this particular sub-bituminous coal, as PRB is one of the lowest 
sulphur content coals in the continental United States. An economical advantage to using bituminous-ranking coals is the fact that bituminous coals are the most abundant forms of coal. [14]. The freshwater origin of the Powder River Basin strata enabled high moisture and low sulphur content - characteristics of low-ranking coals. The carbon content and the variance of the fine ash and carbon content makes characterization of PRB coal and its char non-trivial, necessitating certain approximations and heuristics from a highly-localized sample of coal. Since this study does not have the benefit of eons of compaction and deposition, it is this study's hope to generalize some of the findings towards other sub-bituminous coal (e.g. Pittsburgh-8 and West Virginia coal). Previous research performed on PRB coal char mainly dealt with the gasification processes and the effect it has in reducing global $\mathrm{CO}_{2}$ levels during combustion, especially in conjunction with additives such as $\mathrm{Na}_{2} \mathrm{CO}_{3}$; which serves as a catalytic component to reducing activation energies with gasification reactions. [15] Therefore, it seems that there are many potential avenues using additives to various coals and taking advantage of the lower activation costs due to the dielectric abilities of low-ranking coals such as PRB. Previously, the analysis of the complex dielectric interactions with the coal char and microwave radiation has not been thoroughly studied and should lead to new insights on the manufacturing of granular dielectric in a synthetic fashion.

\section{Computed Tomography}

\section{Background Information}

Tomography is the study of sectioning materials for understanding of the inner morphology and structure of materials. Physical methods of separation can foul the sample being observed, and obviously cannot be performed on people without significant duress invoked, so electromagnetic radiation (commonly in the form of x-rays) are used to "section" the specimen and then mathematically reconstruct the specimen in a digital framework. This is the essence of computed tomography. [16] In the 1930's, Alessandro Vallebona established the 
practice of focal plane tomography, one of the first radiographical techniques. This evolved into the early 1970's with computerized tomography (CT) which Sir Godfrey Hounsfield and Allan McLeod Cormack developed and jointly received the 1979 Nobel Prize in Medicine for their significant contribution to medical science. [17] The field of metrology also developed due to CT imagery, allowing the x-rays to elucidate any defect in the specimen and to measure and detect voids in opaque materials.

\section{Operating Principles}

There are several imaging techniques regarding industrial CT-images. One such method is the line beam scanning method, which involves a collimated beam of x-rays impinging on the sample and the x-ray beam is then swept in a linear-fashion across the sample laterally and rendered via a CAD-neutral file. [18] Cone-beam scanning is another common method of using x-rays to analyze the tomography of a specimen. The x-ray beam shoots a cone over the sample as it is rotated on a rotary table. This then creates 2D-planes via a process called "back-projection", which are further processed via a high-band pass frequency (Fourier) filter to eliminate any back-projection blurring of the specimen. The rectified images are then recompiled into image files called TIFF files which could contain data on over several hundred images stacked together that when rendered in a software, can generate a three-dimensional, complete reconstruction of the sample. This technique is more appropriated in materials science and utilized in generating this study's CT image results using a ZEISS Xradia micro-CT scanner. [18]

\section{Limitations of Technique}

The non-intrusive method of characterization and the accuracy of results make computed tomography (or micro-computed tomography for materials science) one of the goldstandard approaches to analysis of biological and abiological samples. In manufacturing and materials science research, the cost of a micro, x-ray, CT scanner is prohibitive, and 
3D-reconstruction and analysis require vast amount of computing resources and time to adequately resolve the specimen in a digital framework. Larger specimens cannot easily be imaged due to the penetration depth of x-rays being shallow (on the order of decimeters). Image artifacts such as beam hardening and charging (seen on magnetic materials) could complicate image results and machine-learning algorithms and some materials such as quartz or carbonate fossils cannot be readily resolved due to lack of contrast within the specimen

itself. [19] As detailed prior: computed tomographic techniques revolutionized the way humanity interacts with materials, from looking from an outsider perspective, instead peering from within the intricacies of the form and function of the subject; living or abiotic. By simulating a surgeon's knife by using high-energy photons in the form of x-rays, the researcher can "section" the subject piece-by-piece and recombine it in a virtual framework where the potential for interaction with the material is still in its infancy.

\section{Discrete Element Modelling}

\section{Theory}

Physics of the continuum presents a unique, and often unfathomable challenge to numerically solve. The advent of discrete element modelling (DEM) came to pass in the early 1970's via the work of Peter A. Cundall to elucidate geomechanical problems regarding rock physics. [20] This enabled discretization of unique elements that are finite - mathematically "countable", in such a way as to categorically solve non-trivial physical phenomena such as flow, mass transfer, energy transfer, and many-bodied phenomena in a realistic fashion. The discrete element method starts with generating particles with predetermined physical characteristics; this could be size, mass, velocity, direction, and shear strain, chosen in such a way to adequately represent empirical conditions. The discretized model has an initial step, which is then proceeded by time-stepping at a predetermined time value where at each time-step, physical laws are applied to solve problems regarding the interaction between 
the particles or the simulated environment. With many-bodied phenomena common in the industry, this process can be computationally taxing and require vast amount of computer memory. As such, most DEM software utilizes a "nearest neighbor" approach to cut computational cost on a periodic basis. [21] The forces solved for typically utilizes Newton's laws of motion and applies algorithms to integrate the sum of all forces impinged upon these particles, which may range from the macroscopic (friction, gravity, collision), to the microscopic

regime (molecular dynamics, electrostatics, vibrational modes). Integration methods, which are commonly use to simplify ordinary differential equations, are then applied using various algorithms such as Verlet integration and Leap-Frog method. The DEM software utilized in this body of work, Yade, solves these interactions with Verlet integration with further modification done via CAD software Paraview. [22]

\section{Implementation}

From its inception, discrete element modelling (like finite element method and analysis), is commonly used by physicists and engineers to democratize the solving process of the implementation of designs, commonly aided by CAD models. The vast interpretive methods to solve continuum problems can aid in stereology (study of powders), rheology, powder dynamics, and gravitational deposition. Potential drawbacks can be multifold: the computational complexity of many-body problems (millions of particles, large range of distance, etc.); the lack of computer memory allocation, a problem that can hurt economy-of-scale; the commonplace lack of high-end GPUs that may necessitate the need for parallel computing APIs such as CUDA (Nvidia Corporation) via high-performance computing (HPC) environments. [23] 


\section{Finite Element Analysis}

\section{Theory}

As introduced above in the Rationale, finite element analysis (or "method") is commonly used to solve differential equations of high order and non-linearity by partitioning solutions via approximation methods at the interfaces of the constructed mesh and solving boundary-value problems. [9] This technique is exceptionally valuable with regards to analyzing fluid flow, structural deformation, electromagnetic interactions, mass transfer, and any sort of "continuum" problem that can be solved by interpretive (finite) methods of partitioning the problem into boundary value problems commonly encountered in solving higher-order (or non-linear) differential equations. The system is then reduced to problems that as a whole can adequately approach the real solution (by solving each finite element individually), and by applying variational calculus to the resultant solutions, can lead to solutions that approach the theoretical solutions. The singular problems that are to be solved iteratively are segmented virtually by domains, which can each approximate the solution in each of the domains by faceting the physical representation of the geometry of the object. By faceting the geometry of the object (of which could be of high irregularity), into finite triangular domains, the final solution can capture local physical effects that can be difficult to extract from general solutions of the entire geometry. [24]

\section{Implementation}

When the geometry is rendered with these triangular domain facets, a wire-frame or "mesh" is created. This mesh consists of many elements: a vertex at the meeting of the rays traced by the triangles; edges which trace the vertices along the entire geometry; and faces which consists of the triangular facets that envelop the surface of the geometry. Polygons in some FEA solvers can be then be "smoothed" to create surfaces via such methods as finite difference time domain methods (FDTD) [25] or tetrahedron-based meshing finite difference frequency domain (FDFD) approach that COMSOL Multiphysics employs. This 
can be performed using integration methods analogous to DEM software. These domains can then assert various properties given their spacial arrangement, whether a force changes locally (i.e. shear strain) or dissimilar materials/elements converge (i.e. composite materials) where the solution becomes non-trivial at these boundaries. Fortunately, there have been applications of using finite element analysis to model and solve for the interactions between multiple materials and geometries, lending itself to simulation of multiple materials extant in the syn char structure and emulating reality in a statistically-significant way. [26] The mesh that is formed can precisely solve singular elements by utilization of initial-value properties (e.g. temperature or composition of a domain) and reconstitute them into a general solution. These problems then reduce to being algebraic (steady-state problems) to ordinary differential equations (transient problems), of which the software can readily solve without issue. It is beneficial to have a "fine" mesh quality that avoids curved surfaces and to better approximate extreme changes in the geometry of the surface, albeit at an increased computational cost of the software's memory. This leads to studies and research pertinently achieving a state called "mesh independence", in which the final solution does not change in a non-negligible way and thus be considered "mesh-independent". Since a perfect solution does not exist unless there are an infinite number of segmentation, error reduction is applied to smooth regions using polynomial methods (Euler and Runge-Kutta) to enable the solution to converge with reasonable certainty. Integrative methods include Gaussian quadrature and use of the mathematical Jacobian to describe movement and flow of nodes in a mesh. As such, any contemporary analysis on continuum physics now use finite element method and analysis techniques to solve problems that years before could not be readily computed without great difficulty and time. With this particular study, large swaths of computing time was placed on the electromagnetic physics module, specifically the radio frequency $(\mathrm{RF})$ frequency domain solver, which iteratively solves Maxwell's equations using sinusoidal wavefunctions that sum up to a numerical approximation of the wave equation. The wave equation is a non-linear partial differential equation (PDE) with no exact solu- 
tion, such that converging to a realistic solution involves numerical methods to approach the analytical (infinite) solution. It is also relevant to recall that the wave equation and its solutions are where Maxwell's equations were originally derived. [27] The RF module itself contains a bevy of equation solvers which that can be employed to accurately depict RFbased applications such as antennae, scattering phenomena, microwave reactors, millimeter and terahertz-based waveguides, and other wave-based phenomena. FDFD's true strength is elucidating the frequency-dependent material properties under electromagnetic radiation, as the frequency heavily influences the scattering parameters, and subsequently the absorption of EM waves. 


\section{CHAPTER 2}

\section{RESULT AND DISCUSSION}

\section{Methods of Materials:}

\section{Preparation of Computational Analysis of PRB Coal Char}

Extensive use of computational software was required to facilitate the simulation and its relevance to the reconstruction of the microwave reactor with high fidelity. Coding of macros used in generating plots and figures, and interpolation using the prescribed NRWmethod was done in MATLAB and Python. Yade and Paraview were used to generate and manipulate the virtual coaxial transmission line with particle deposition and packing primarily done by Yade and Paraview exporting the .stl files. Image analysis was performed using ImageJ (Fiji) and various additional Java plugins were utilized to enable proper quantization of particle density and count; as well as ascertaining volume fraction. Finite-element analysis was performed by COMSOL and used the electromagnetic waves and electrostatic physics modules.

\section{ImageJ and Paraview Analysis}

To properly use Yade to model the spheres in accordance with empirical properties of the coal particles; image analysis using Paraview and ImageJ was utilized. After the necessary thresholding of the CT scans, the particles were analyzed using embedded plugins available in ImageJ to obtain an approximate count of particles and areas per slice of CT scan. Since sieve analysis of the particles was used prior to CT scanning, one could reasonably assume a Gaussian (normal) distribution of diameters centered around the mean value of the diameter. A snapshot of a portion of the TIFF stack of images of the x-ray CT scan is shown in Figure 2.1. 


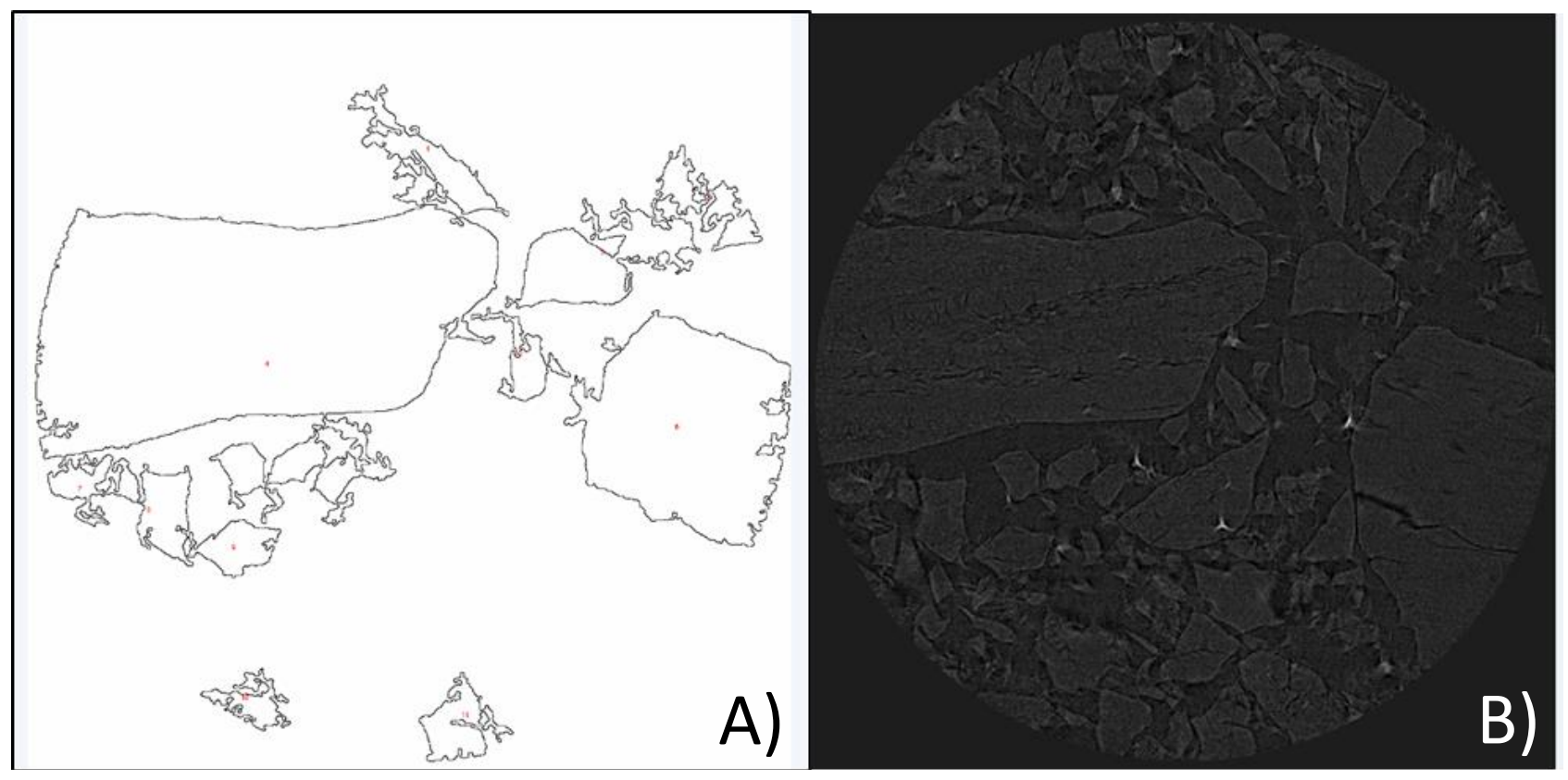

Figure 2.1 Volume reconstructed data of PRB Coal taken using micro-CT machine. Subfigure A illustrates the selection of particles edges using ImageJ. Subfigure B is a slice of the raw reconstructed CT image in ImageJ. The diameter of the circular image is approximately 1108 microns in diameter.

Care was taken in assuring that only rigid particles roughly approximating a polygon were counted, as well as using embedded plugins for ImageJ, to check for circularity. A technique in stereology called Feret's diameter (commonly known as the caliper diameter) was used to approximate the actual diameter of each counted particle, thereby ensuring that the waxing and waning of each coal particle is accounted for by taking the greatest distance between two separate, usually subsequent, 2D-slices and measuring the angle between them (called the Feret angle). This average is used in powder analysis which is considered to be germane to this field of study since the subject is powder/crystalline-like and thereby a suitable candidate in approximating the diameter of each coal particle. [28] The resultant histogram is detailed in Figure 2.2, where the Feret diameter appears to skew right around 200 microns.

As shown in Figure 2.2, the value of Feret's diameter ranges from as low as 80 microns 

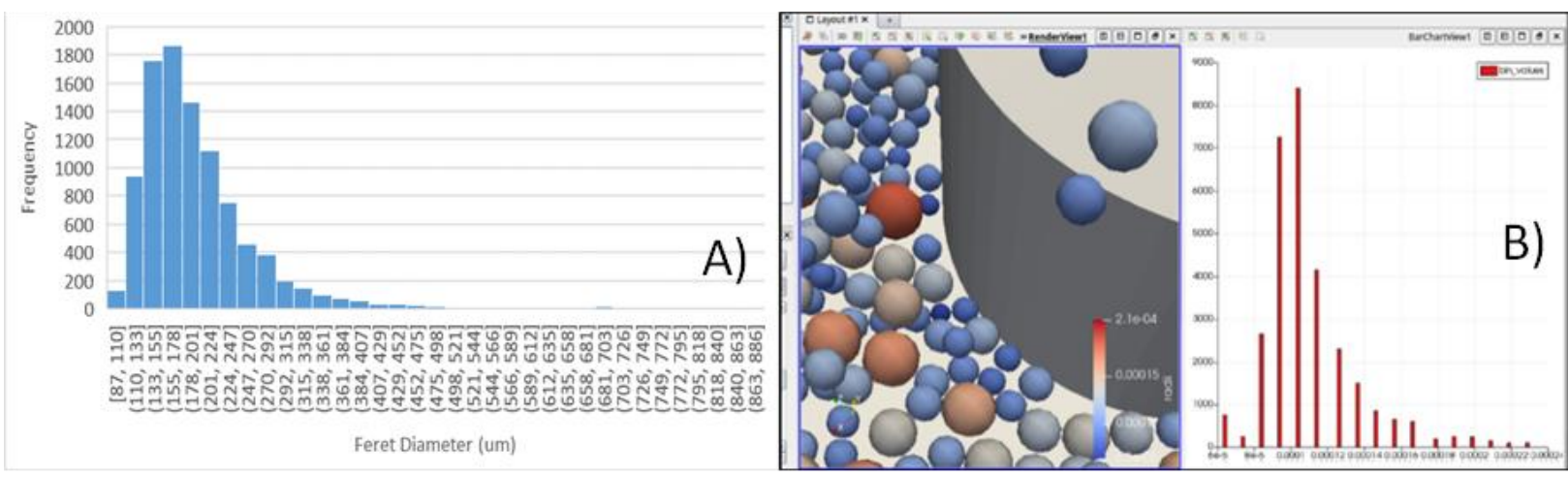

Figure 2.2 Subfigure A is histogram of the particle diameter (Feret diameter) of the particles taken CT images of PRB char. It is noted that the distribution is skewed. Subfigure B is an image the DEM simulation of particle filling and the instantaneous histogram of particle diameter. Note the similar skewed distribution between A and B.

to several particles over half a millimeter (500 microns). The average of the results in this histogram point to a mean value of 197 microns. This analysis was repeated using the summary of each TIFF slice and yielded similar results, centering in a mean value of 210 microns. Taking these values, a particle size distribution (PSD) was created in Yade to model a realistic normal distribution of spherical particles simulating the observed PRB coal char particles and thereby enabling proper viewing of the dielectric and magnetic properties of the particles using Paraview and COMSOL. This can be non-trivial with regards to 2dimensional slices of a 3-dimensional object, as the diameter of each coal particle waxes and wanes accordingly over the TIFF stack of files.

\section{Discrete Element Modeling}

Yade is the discrete element method software employed to generate the particle filling geometry of the micro-reactor. The software is an "extensive Open Source GNU/GPL Software framework" designed with dynamic libraries in a way that it is easy to add new numerical models for simulation. [22] The software has a complete modeling framework built and used extensively for particle compaction and deformation of spherical bodies when var- 


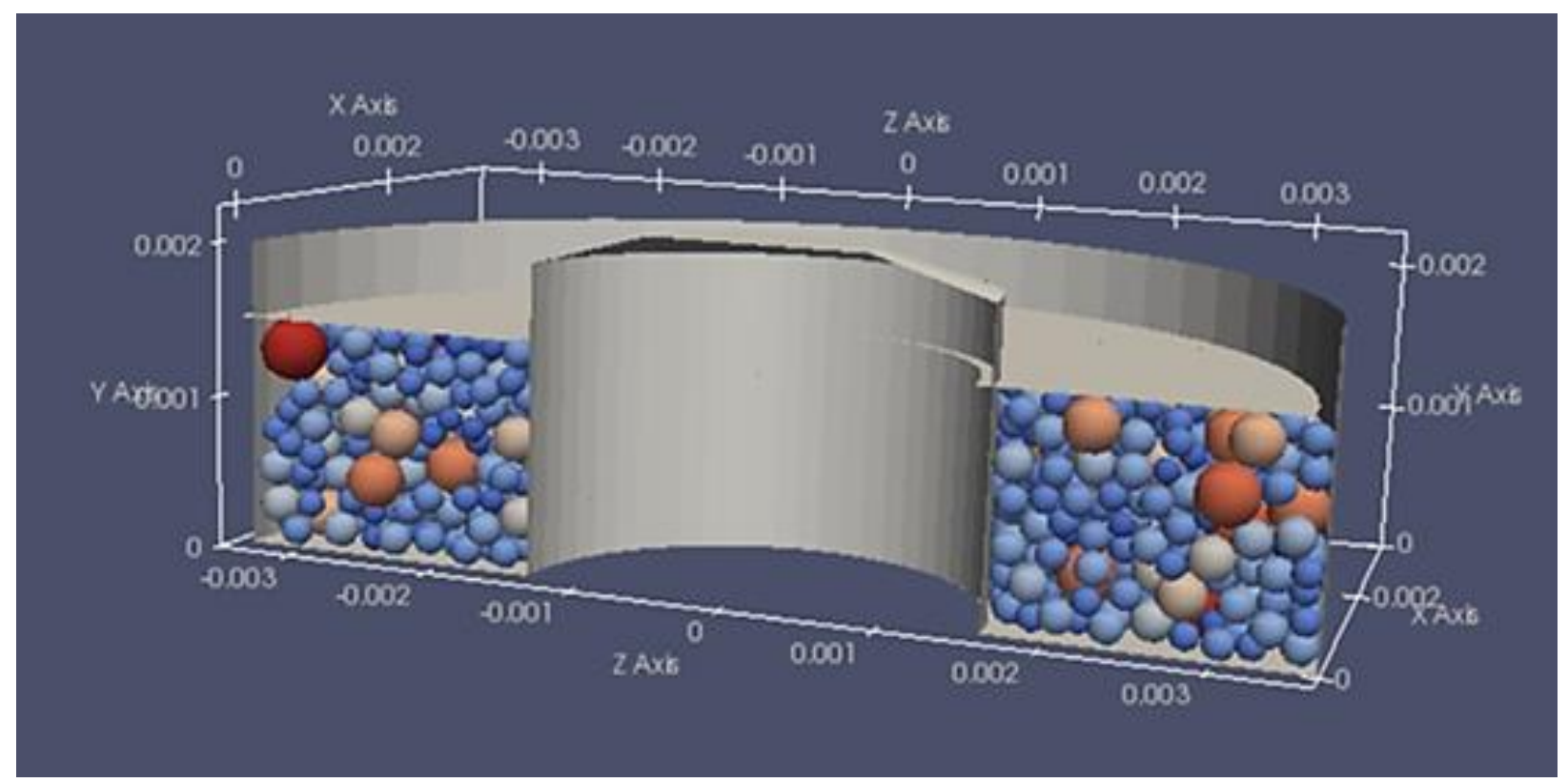

Figure 2.3 Illustration of a DEM-simulation of char where the particles are approximated as spheres. A coaxial test cell is being filled and compressed in the simulation. The test cell has an outer diameter of $7 \mathrm{~mm}$ and inner diameter of $3 \mathrm{~mm}$. The particle diameters are based on the distribution of particles sizes from the micro CT images.

ious stresses and environmental geometries are present, such as the physical compaction process of hydrocarbons in a mineral substrate formation. Many-body simulations, such as the particle filling geometry model, fits perfectly within Yade's purpose and is apt for this study. Figure 2.3 is an illustration of the particles filling a coaxial sample that has an outer diameter of $7 \mathrm{~mm}$ and an inner diameter of $3 \mathrm{~mm}$ in accordance with the coaxial airline geometry used to analyze the actual coal char sample. This filling motion was then coupled with a ramming press to compact the spheres in a naturally occurring manner. This is to mimic the compression tools that are commonly used to stabilize, and to prevent leakage, in coaxial cables such as those used in VNA. The physical stresses and deformation information gathered by the software was encapsulated into a CAD-neutral file and exported to Paraview for volume fraction analysis and import into the COMSOL software. 


\section{Volume Fraction Analysis of PRB Coal Char}

In order to ensure that the DEM simulation is representative of the real PRB coal char, care was taken to verify the volume fraction of the PRB using the CT image analysis garnered from the ImageJ analysis software. Also needed was the total number of particles in the particle deposition model, which was visualized using Paraview as seen in Figure 2.3.

\begin{tabular}{|c|c|c|c|c|c|}
\hline \multicolumn{2}{|c|}{ Image CT Analysis } & & & & \\
\hline \multicolumn{2}{|c|}{ Volume Fraction } & \multicolumn{4}{|c|}{ Yade (DEM approach) } \\
\hline Voxels in stack: & $942,961,500$ total voxels & \multicolumn{2}{|c|}{ Yade (getDepthProfile Fill) } & \multicolumn{2}{|c|}{ Yade (getDepthProfile Ram) } \\
\hline Voxel size: & $1.15 \mu \mathrm{mx} 1.15 \mu \mathrm{m} \times 1.15 \mu \mathrm{m}$ & Section & Volume $\left(\mathrm{m}^{3}\right)$ & Section & Volume $\left(\mathrm{m}^{3}\right)$ \\
\hline Thresholded volume: & $6.437 \mathrm{E}-13 m^{3}$ & Total & $3.287 \mathrm{E}-08$ & Total & $2.592 \mathrm{E}-08$ \\
\hline Average volume/slice: & $6.602 \mathrm{E}-16 m^{3}$ & Volume (cylinder) & $7.854 \mathrm{E}-08$ & Volume (cylinder) & $4.398 \mathrm{E}-08$ \\
\hline Total ROI volume: & $1.436 \mathrm{E}-12 m^{3}$ & Fill & 0.4185 & Ram & 0.5893 \\
\hline Volume of Stack: & $1.444 \mathrm{E}-09 m^{3}$ & & & & \\
\hline
\end{tabular}

Table 2.1 Comparison of experimental volume fraction analysis and DEM results for fillingand after-compression. The left table is the experimental volume fraction inferred from the CT images at $44.82 \%$. The right table refers to the DEM simulation in Yade with $41.85 \%$ volume fraction when filled (Fill) and $58.93 \%$ volume fraction when compressed (Ram).

As described earlier, the DEM simulation both filled and compacted the coaxial airline using a ram cycle that was added to the gravity deposition model to eliminate any excessive void space. This was needed to replicate the natural packing procedure for preparing the experimental coal char in lab for VNA measurement. In addition to pressing the coal char particles, as seen in Figure 2, the volume was vibrated to aid in the packing. Using the particle size distribution (PSD) generated from ImageJ and the thousands of spheres with varying diameter and their interactions under gravity and compression, a summary of the volume fraction of PRB can be seen in Table 2.1.

Using the voxel counter plug-in in ImageJ, it is possible to find a volume fraction (see the left portion of Table 2.1). This was done by knowing the total field diameter of approximately 1108 microns. Then extracting the total volume of the TIFF stack by using binary thresholding, where ' 1 ' details regions of interest (particles) and ' 0 ' detailing void 
spaces. From this, the CT images can give us a rough estimate of the volume fraction. To confirm that the same volume fraction was obtained from the DEM simulation the "getDepthProfile" function in the Yade software was utilized. The right portion of Table 2.1 highlights the DEM volume fraction for both the "Fill" and "Ram" operations, which are $41.8 \%$ and $58.9 \%$, respectively. This is realistic given that the "Ram" simulation should have eliminated unnecessary void space. The finalized DEM geometry was then imported into COMSOL for further analysis of the PRB char.

\section{COMSOL Simulation}

The bulk of the computational work was done using COMSOL Multiphysics (version 5.6) and the RF module in solving for scattering parameters and conductivity by solving highly-nonlinear Maxwell's equation over thousands of domains and facets with the edges of the coaxial airline employing boundary conditions akin to the airline dimensions used during the experiment [27]. A custom macro was utilized to separate the void space in-between the particles of the simulation and allow for optimal meshing on which solving the non-linear PDEs occurs. Each sphere is a dielectric analog to its real counterpart - referencing established permittivity and permeability values, as well as electrical conductivity properties of the compounds illustrated in Table 2.2 and asserting said properties using a separate random number generator $(\mathrm{RNG})$ code developed to give the approximate proportions of particles occurring in real PRB coal char samples. Random number generators uses pseudo-random properties, such the local time on a computer server, and allocates an algorithm to introduce more randomness in selecting numbers. The primary purpose is to eliminate arbitrariness seen from contrived numbers from operators or repeated measurements/simulations. This pseudo-random algorithm (enacted using a randomizer function in Python), can help to eliminate "striping" or grouping of the particles in the simulation and to make the simulation as naturalistic as possible. The random number generator code was written in Python 3.1 and is found in the Appendices. 


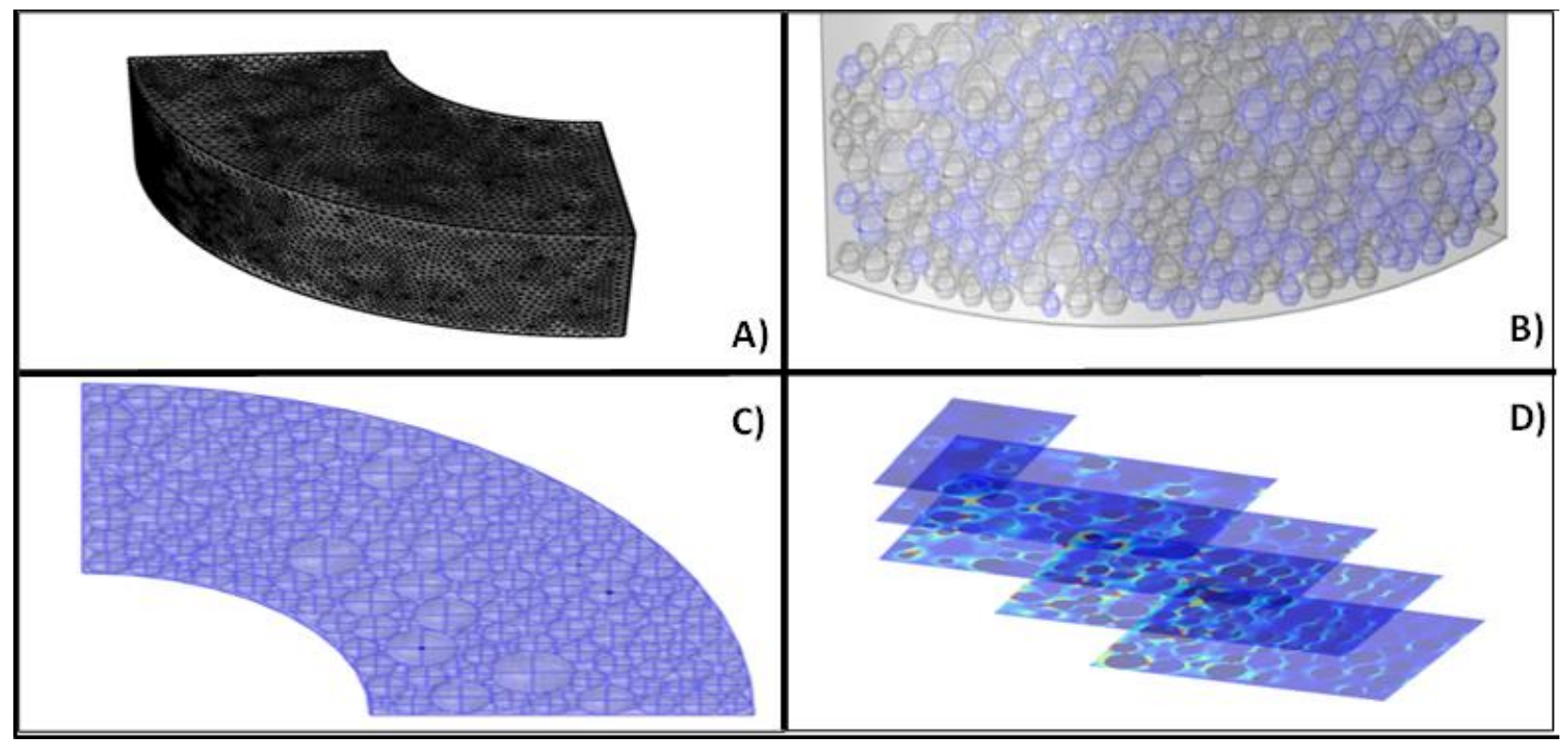

Figure 2.4 Images from COMSOL RF simulation. Subfigure A is a physics-based mesh for the electromagnetic wave study. Subfigure B is a selection (highlighted in blue) of individual particles in the simulation based on RNG macro. Subfigure C is top view of packed of the spheres with varying radii. Subfigure $\mathrm{D}$ is a multi-slice with electric field color contours. Note the enhanced electric field between particles that highly influence dielectric response in granular dielectric materials.

The COMSOL simulation has proven to be easily repeatable and tunable, enabling simulations to finish with complete results within several hours (full 1-10 GHz microwave spectrum). The analysis of the dielectric parameters was performed using a de-embedding code that has been modified to extract dielectric properties based on scattering parameters obtained and is optimized to ensure accuracy to the original NRW-method paper according to Weir et al (1974). [29] The Nicolson-Ross-Weir method, as previously described in the Introduction, is a technique to ascertain the relative electric permittivity and the relative magnetic permeability, in which "relative" pertains to the associated vacuum values of both electric and magnetic fields. The NRW code is located in the Appendices under "NRW Polynomial Interpolation Code" and takes the scattering parameters (in .csv file format) and solves for the reflection coefficient. The reflection coefficient $(\Gamma)$ is the measure of how much a wave, in this case, microwaves, are reflected upon a discontinuity in impedance. [30] 
The NRW method loses precision at half-wavelength regions such that the code utilized in the study was modified to have a polynomial fit to these problematic regions (lines 36 to 48 in NRW interpolation code). This polynomial fit is the same fitting algorithm also utilized in FEA meshing processes, i.e. using Euler and Runge-Kutta polynomial approximations. Fortunately, the reassignment of the property of the spheres can be done with ease, including adding more material properties if increased robustness is desired. It can be shown that the results are very consistent given the RNG code's shuffling ability of asserting certain properties of the spheres in the simulation and over numerous trials. Figure 2.6 shows us a suite of images taken of the COMSOL simulation.

\section{COMSOL Modelling with PRB Coal Char}

The COMSOL simulation was set up by importing the Paraview model that was used for volume fraction analysis and then constructing a virtual coaxial airline within the COMSOL interface akin to the airline used in the experimental measurement of dielectric properties. Due to the sheer amount of data processing involved, quarter-symmetry of the cylindrical airline was taken advantage of to ensure that each simulation can be repeated efficiently to avoid wasting significant amounts of processing time. A random number generator was programmed to assert properties of ash content and carbon content (minus the volatile content) using feedstock values of the Powder River Basin sub-bituminous coal taken from prior EDX reporting at the National Energy Technology Laboratory (NETL) in Morgantown, West Virginia. [31] The feedstock weight percent values of the dried PRB coal utilized in the simulation are seen in Table 2.2 with the RNG code assigning a value for picking the particles in the simulation via their respective amounts. Many of the smaller ash content compounds such as those less than several weight percent (wt\%) were omitted in this current COMSOL simulation. Of distinct importance is the ultimate analysis weight percent of carbon being $70 \%$, which was split into several observed allotropes of elemental carbon in the char, with the other $30 \%$ being allocated to the existence of ash remaining after the coking 
process. It is assumed in the simulation that the moisture and all volatile organics have been driven-off during the pyrolysis process and therefore additional variation of the ash particles is employed to counteract the loss of weight percent due to desiccation. Even though the tradition VNA measurement suspends the coal char particles in paraffin, the resultant DEM model and subsequent analysis via FEA has air in the interstitial voids between particles for computational fidelity. In order to ascertain what type of carbon content primarily occurs within the torrified PRB coal sample, it was beneficial to study the Raman shifting of the dried PRB. Due to pyrolysis to devolatilize the sample, it was noted that there was a substantial increase in sp3-hybridized carbon (amorphous carbon) as opposed to graphitic sp2-hybridized carbon allotropes. This hypothesis was initially devised by looking at the CT images that revealed distinct puffiness mimicking amorphous carbon (hence the assertion that the primary carbon content is amorphous and therefore sp3-hybridized carbon) the where it is assumed that all of the volatile organics and moisture are driven-off at $500^{\circ} \mathrm{C}$ over the long holding time. This created a porous structure similar to coking, although PRB coal is chosen such that coking is minimized in the process. We have allocated a majority of the weight percent of the carbon content (85\%) towards amorphous carbon (carbon black) and $15 \%$ graphitic carbon. All of the remaining ash content, assuming roughly $30 \%$ after all the volatile organics driven-off, is detailed within.

\section{Results and Discussion:}

\section{Density of PRB Coal Char and Synthetic Char}

For an accurate model of a generic, sub-bituminous coal char sample, it would beneficial to understand how density may play a role in the dielectric properties of the synthetic coal char and how it relates to the real sample. The experimental portion of the research group determined the density of unpacked PRB coal char to be $1.224 \mathrm{~g} / \mathrm{cc}$, measured in a cylindrical vessel of diameter $0.809 \mathrm{~cm}$ and height $0.631 \mathrm{~cm}$, with mass measured to be 0.1264 grams. This gives the PRB coal char density to be $0.390 \mathrm{~g} / \mathrm{cc}$. This is in agreement 


\begin{tabular}{|c|c|c||}
\hline \multicolumn{3}{|c|}{ Carbon Content (70\% ultimate wt\% of synthetic char) } \\
\hline Amorphous carbon & 85 & $\mathrm{wt} \%$ \\
\hline $\begin{array}{c}\text { Graphitic Carbon } \\
\text { (pyrollized) }\end{array}$ & 15 & $\mathrm{wt} \%$ \\
\hline \multicolumn{2}{|c|}{ Ash Content $(\mathbf{3 0 \%}$ ultimate wt\% of syn thetic char) } \\
$\mathrm{wt} \%$ \\
\hline Alumina $\left(\mathrm{Al}_{2} \mathrm{O}_{3}\right)$ & 17.36 & $\mathrm{wt} \%$ \\
\hline Quartz $\left(\mathrm{SiO}_{2}\right)$ & 37.50 & $\mathrm{wt} \%$ \\
\hline Magnetite $\left(\mathrm{Fe}_{2} \mathrm{O}_{3}\right)$ & 3.88 & $\mathrm{wt} \%$ \\
\hline $\begin{array}{c}\text { Magnesium } \mathrm{Oxide}_{2} \\
(\mathrm{MgO})\end{array}$ & 4.68 & $\mathrm{wt} \%$ \\
\hline Sodium Oxide $\left(\mathrm{Na}_{2} \mathrm{O}\right)$ & 5.95 & $\mathrm{wt} \%$ \\
\hline Calcium $\mathrm{Oxide}(\mathrm{CaO})$ & 15.30 & \\
\hline \hline
\end{tabular}

Table 2.2 Initial synthetic char volume fraction that reproduce the dielectric response of actual PRB char. These volume fractions were determined using a combination of COMSOL simulations, Raman spectroscopic analysis of graphitic carbon bands, and visual determination based on stereological techniques.

with the two synthetic coal char densities of $0.5573 \mathrm{~g} / \mathrm{cc}(<150 \mu \mathrm{m}$ diameter $)$ and 0.7341 $\mathrm{g} / \mathrm{cc}(200-300 \mu \mathrm{m}$ diameter). The literature on PRB parent coal has densities of roughly $1.20 \mathrm{~g} / \mathrm{cc}$ and the processed coal has particle densities ranging from 0.7-0.8 $\mathrm{g} / \mathrm{cc}$ assuming greater than $90 \%$ coal conversion during pyrolysis [32]. Density discrepancy is due to lack of eons of mechanical pressure in creating actual coal specimen - synthetic coal char was only compressed by dye in contrast, enabling the possibility of void space to influence overall density of the synthetic char facsimile.

\section{Verification of COMSOL-simulated PRB Coal Char}

Referring to the electromagnetic properties of dried PRB; Singh et al. [33] postulated that at roughly $95 \%$ of power at $2.45 \mathrm{GHz}$, epsilon real approaches 2.65 . The electric permittivity $\left(\epsilon^{\prime}\right)$ is determined to be between 2.5 and 2.75 based on VNA measurements conducted at NETL [34]. Results using this formula of synthetic char obtain permittivity values within $+/-5 \%$ of $\epsilon=2.57$ value computed with VNA with volume fraction stated in 


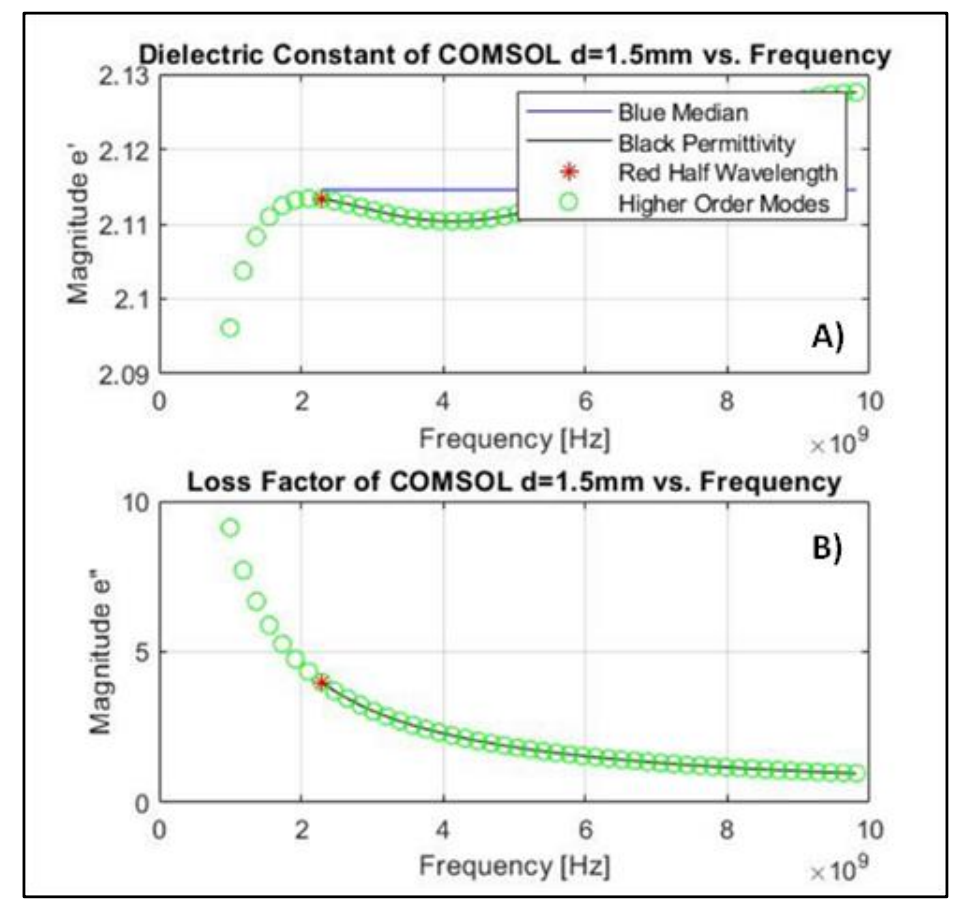

Figure 2.5 Calculated COMSOL permittivity values using the NRW method as a function of frequency from 1 to $10 \mathrm{GHz}$. These values are the synthetic char material outlined in Table 2.2.

Table 2.2. It was noted that it is difficult to determine the complex permittivity values (the 'lossiness' of the dielectric medium) from the simulation. This was reasoned to be due to the NRW to accurately determine imaginary contribution when the medium has moderate electrical conductivity.

The COMSOL simulation yielded comparable results to the VNA performed on the actual PRB coal char for the effective dielectric. The current determination of the dielectric parameters using the more robust NRW method code yielded a relative permittivity value of roughly 2.12 (shown in Figure 2.5) whereas the NETL VNA probe yielded values of epsilon between 2.5 to 2.75 where $\epsilon^{\prime}=2.57$ was the target of the COMSOL simulations and literature values were placed around $\epsilon^{\prime}=2.6$ for the dielectric constant of PRB coal char [33]. This may be due to the interpolation this code provides (i.e. smoothing and the forcing of the halfwavelength anomalous region) which could drive the true permittivity value slightly lower 
than recorded but more consistent overall and has been an issue previously discussed in the literature [35].

\section{Examination of Fitting Scattering Parameters Using a Modified Approach to Weir et al. (1974)}

A common problem into translating Weir's work into Python code was failing to to realize the discrepancy at half-wavelength regions due to destructive interference patterns where the waveform drastically diverges into half-wavelength. These periodic impulses can perturb the convergence and therefore damage the accuracy of the NRW code. Therefore, a new and robust code was modified that could smooth these problematic regions and could give us better numerical precision than the previous code mentioned above. The smoothing is achieved by fitting a polynomial to the data as illustrated in Figure 2.6A. The research into the NRW method from Vicente et al. [36] claims that ambiguity of the phase of the waveform (seen in Figure 2.6C, which highlights a substantial phase divergence as frequency increases) can be due to magnetic and conductive materials that are "lossy" (defined by the imaginary terms of the S11 and S21 parameters).

It has also been noted within the literature [36] that air gaps and voids could be problematic in obtaining deviation of values of the magnetic permeability, which persists even in the smoothing code now employed. Forcing the permeability of the constituent particles in the COMSOL simulation, unfortunately, did not assuage the divergence as expected, so this may be due to the presence of iron oxide and the omnipresent air gaps that permeate the airline which is accounted for in the simulation but may lead to these impulses to appear. Xu et al. [35] used a similar method to obtain permeability values that should range between 1.0 and 1.1 for $\mu^{\prime}$ and -0.1 to 0.1 for $\mu^{\prime \prime}$ with similar loss tangent values as what we have obtained. Also noted is the gamma term (one minus the transmission value in Figure 2.6C) being near 0.7 which is a value purported with $\mathrm{Xu}$ et al. and several other papers [5] involving coal char dielectric determination. Furthermore, as noted in Figure 2.6D the phase is dependent on 


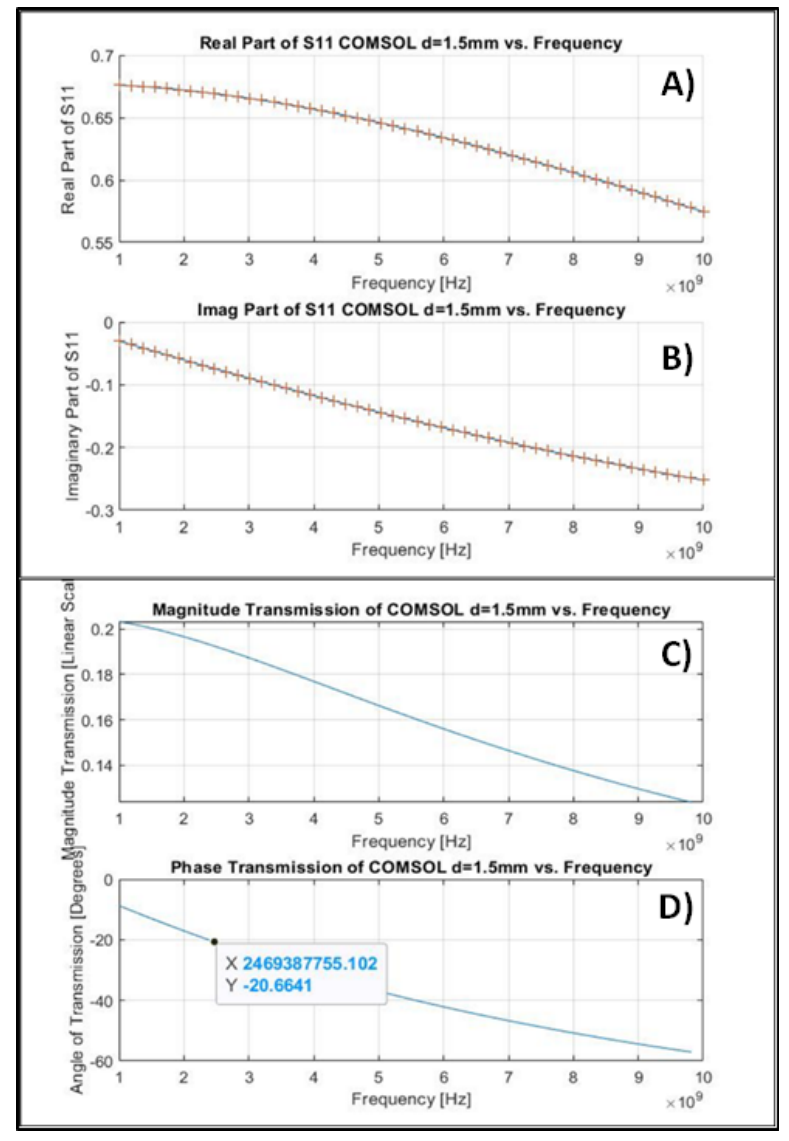

Figure 2.6 Polynomial fit of real (A) and imaginary (B) components of S11 calculated in COMSOL for synthetic char material. Subfigure C and D are the corresponding transmission magnitude and angle. Note the phase dependence with frequency.

the frequency, which further supports the complex dielectric interaction of the mixture and losses occurring. The material attribute that has been not extensively matched but could be significantly contributing to this phase dependence is the electrical conductivity, which will be discussed in the next section.

\section{Modification and Analysis of Conductivity of sp2-hybridized Carbon Species in Synthetic PRB Coal Char}

Due to the large phase dependence of the synthetic char and lack of empirical evidence of electrical conductivity of three-dimensional coal char samples, research was performed on 


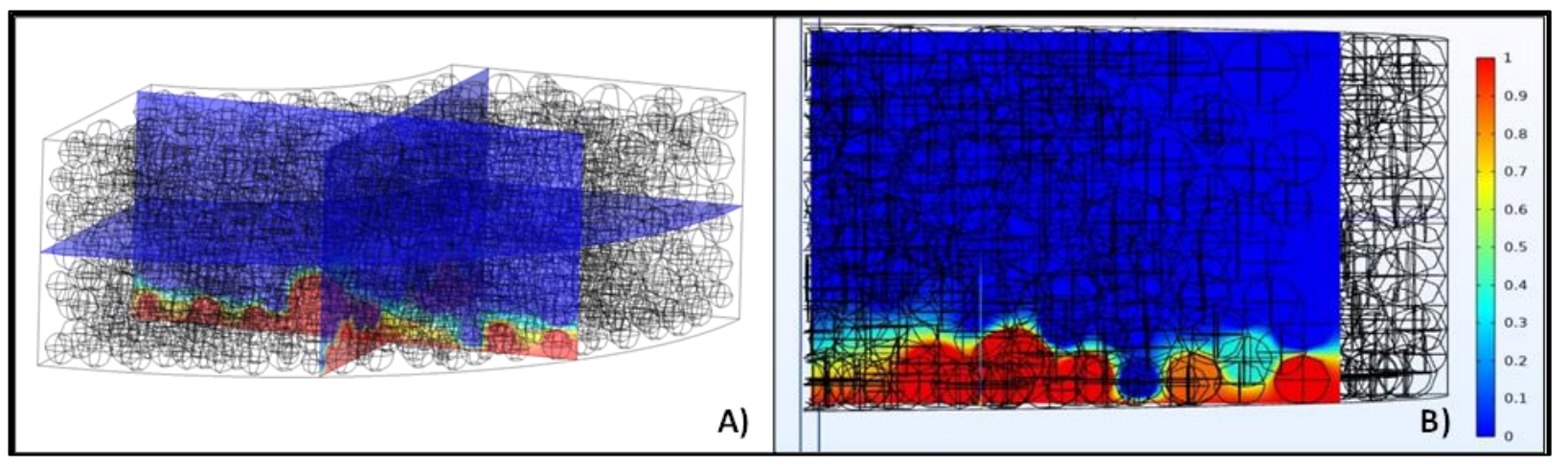

Figure 2.7 Illustration of the voltage distribution in an electrostatic simulation of synthetic char. The bottom boundary has a voltage of $1 \mathrm{~V}$ and the top is grounded. Notice that the conductive particles closest to the voltage boundary conduct the voltage until they interface with a less conductive particles. The overall current will depend on the network of conductive particles within domain.

elucidating the proper ratio of sp2-hybridized, pyrolyzed, graphitic carbon to amorphous carbon and its overall effect on electrical conductivity. From literature [37], it is known that graphite is several orders of magnitude more conductive than its amorphous allotropic counterpart, such that our previous formula of $15 \%$ graphite weight percent out of the $70 \%$ total carbon weight percent in the simulated coal char had to be modified to ensure that the conductivity is comparatively reduced to the PRB coal char. An electrostatic calculation was conducted using the $\mathrm{AC} / \mathrm{DC}$ module within COMSOL Multiphysics using the same geometry as the RF study. To expedite the calculation of the DC, time-invariant electrical properties of the synthetic PRB char as a rough estimate to enumerate conductivity values not usually undertaken in microwave reactor catalysis experiments. There are roughly 1023 spherical particles in the quarter-symmetry model, with 600-700 particles being graphite and amorphous carbon and the other 300 particles allocated to the ash content of the coal char corresponding to Table 2.2. Again, the assignment of properties to individual spheres within the domain was done using a random number generator and several trials to achieve an average conductivity value.

The current-voltage (IV) curve was calculated from the electrostatic calculation and 


\begin{tabular}{|c|c|c|c|c|}
\hline Trial Seed & $0 \%$ Graphite Load $(\mathrm{S} / \mathrm{m})$ & $5 \%$ Graphite Load $(\mathrm{S} / \mathrm{m})$ & $10 \%$ Graphite Load $(\mathrm{S} / \mathrm{m})$ & $15 \%$ Graphite Load (S/m) \\
\hline 1 & 0.0015 & 0.0118 & 0.0270 & 0.0267 \\
\hline 2 & 0.0006 & 0.0286 & 0.0152 & 0.0248 \\
\hline 3 & 0.0010 & 0.0279 & 0.0181 & 0.0115 \\
\hline 4 & 0.0011 & 0.0214 & 0.0383 & 0.0280 \\
\hline 5 & 0.0013 & 0.0214 & 0.0180 & 0.0202 \\
\hline Average & 0.0011 & 0.0222 & 0.0233 & 0.0222 \\
\hline
\end{tabular}

Table 2.3 DC electrical conductivity values calculated from COMSOL-derived IV curves. Present are outlier values; it can be inferred that particle placement supplanted graphite load for contribution towards conductivity. Conductivity depends on conductive network of particles so several values were conducted and averaged. Conductivity plateaus after $5 \%$ loading up to $15 \%$

the electrical conductivity was determined. Figure 2.7 is an illustration of the spatial variation of the voltage for one trial. The voltage boundary condition is applied to the bottom of the domain the top of the domain is grounded. Notice in Figure 2.7A that the voltage at the bottom is readily conducted through the particles at the bottom of the domain that are electrically conductive. The voltage quickly decreases at the interface with less conductive particles. The overall current between boundaries ultimately depends on the network of conductive particles. It was determined it was necessary to take several trials where the material assignment was randomized to determine an average conductivity value. A select number of these trials are provided in Table 2.3 for several graphite loading cases.

Table 2.3 used numerous COMSOL trials of several different weight fractions to see how much graphite can modify the conductivity. The results demonstrate a non-negligible amount of randomness in conductivity that can change based on the RNG seed and particle allocation; only when numerous trials at each loading were undertaken could a clearer trend of the results be observed. The random sampling of sphere placement in these trials should therefore indicate how the conductive properties of the synthetic char relate to the networking structure of conductive particles as Figure 2.7 readily illustrates with the conductive networking between the spheres. The COMSOL model trials in Table 2.3 are more robust but conveys the overall same magnitude of conductivity above $5 \%$ graphite (sp2-carbon) loading. It is important to note that there was initially compression in the DEM model 
from the Yade software as the resultant compression and geometry values were imported into COMSOL using discrete element modelling. It was noted from the accompanying DC measurement that the conductivity values are sensitive to the amount of compression. It was determined from the experimental four-point measurement that the conductivity value for amorphous carbon particles less than 150 microns are roughly $196.1 \mathrm{~S} / \mathrm{m}$, where the conductivity value for particles within the range of 200 to 300 microns is roughly $370.4 \mathrm{~S} / \mathrm{m}$. This confirms that there is an electrical dependence on both the size of particles, a network of particles, and compression of particles. It can be shown from these initial COMSOL calculations that packing density and size correlate conductivity can be corroborated with literature results from Yin et al.'s [37] research on analyzing continuity and electrical conductivity of coal-based films. Yin's conductivity values demonstrated a range of conductivity on the order of 7 or 8 magnitudes $\left(6.25 \times 10^{-6} \mathrm{~S} / \mathrm{m}\right.$ to $\left.1.36 \times 10^{2} \mathrm{~S} / \mathrm{m}\right)$. Amorphous structures - and therefore, discontinuities in the crystalline structure tend to insulate, rather than conduct, electromagnetic radiation. Annealing of coal-based films from Yin et al's work [37] noted that annealing temperatures increased conductivity despite the overall increase of the overall crack ratios of the coal-based film (where you would reasonably expect a decrease in conductivity) exceeding $9.75 \%$ of the sample surface at temperatures around $800^{\circ} \mathrm{C}$. The compression of the DEM model and the compression in our sample for DC measurements of conductivity may override the cracks in the coal char-based film and create a structural conformation that promotes such conductivity, despite some cracking occurring in the pyrolyzed product. Further postulation and discussion on how discontinuities in the surface of a coal-based film could influence electrical conductivity are discussed in the section titled "Analysis of Compression on the Electrical Conductivity of Synthetic Coal Char".

\section{Optical Analysis of PRB coal char versus Synthetic Char}

In order to test the veracity of the synthetic coal char and the deviation of electrical conductivity of both samples, efforts were made to visualize the specimen using optical 


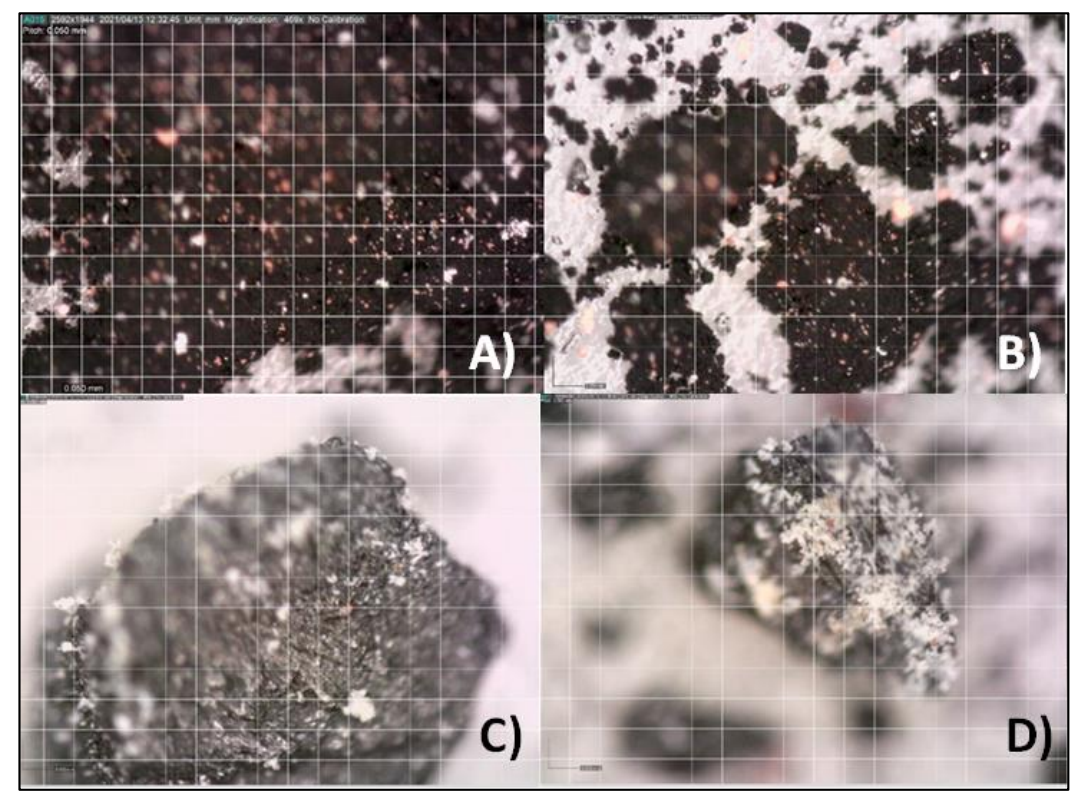

Figure 2.8 Optical micrographs of synthetic coal char (A-B) and actual PRB coal char (C-D). The black material in all the micrographs is carbon. The white material is ash and the red particles are hematite. Note the red particle in Subfigure C for the actual char. Moreover, it was noted that the ash content collects on the surface for actual char (C-D).

microscopy. The synthetic coal char was mixed mechanically with the compounds detailed in Table 2.2 and pressed using mortal and pestle into a fine powder using amorphous carbon and graphite (which themselves were sifted to varying sizes of particles 200 to 300 microns in diameter to particles less than 150 microns in diameter in order to match the CT image scale of actual PRB). The resultant mixture was fired at $900^{\circ} \mathrm{C}$ for an hour. Figure 2.8 are the corresponding microscopy images for both the synthetic char (Figure 2.8A and 2.8B) and the actual PRB coal char (Figure 2.8C and 2.8D). There is a noted similarity between the synthetic and actual char samples, which is to be expected. This is reasoned based on the fact that effective dielectric properties were confirmed to be similar and there are dependent on the largest constituent material, carbon. The black material in Figure 2.8A-D is the carbon material. The red particles are hematite, which was included in the synthetic mixture and are present in actual char. A close look at Figure 2.8C reveals the red particles on the surface of the carbon material. The noted difference between the synthetic char 
and the actual char is the white particles. The white particles are the ash content that undergoes some transformation during pyrolysis. As seen in Figure 2.8D the white ash content is collected around the outside of the char particle. It is hypothesized that this thin coating of ash content is acting as an electrically insulating layer around the particles. The synthetic coal char images detailed in Figure 2.8A and 2.8B appear rather amorphous at the macroscopic level but at microscopic resolution, appears to be a disorderly, yet cohesive,

agglomeration of synthetic coal char with some coordinated framework. Research is ongoing to adhere a fine ash coating to said particles and to test the influence of the coating on the electrical conductivity. The decrease in electrical conductivity of the synthetic char material will subsequently bring the imaginary part of the permittivity within agreement with the actual char.

\section{Further Modification of Synthetic Coal Char to Increase Fidelity of Actual PRB Coal Char}

To simulate actual PRB coal char and to analyze the constitutive effects the ash particles have on the synthetic material as whole, the syn char sample was further modified to bring about better congruence with the actual PRB coal char with its simulated form. In the original PRB coal char XRD, it was shown that the synthetic coal char required a modification of the volume fraction load of graphite from $15 \mathrm{wt} \%$ to $30 \mathrm{wt} \%$ to further simulate reality. As such, the second rendition of synthetic coal char has shown to have increased fidelity to the actual composition of PRB coal char as noted in the comparison of each spectra shown in Figure 2.9. The process involved coating the syn char in a fine ash layer (primarily quartz and alumina) to accurately show the granular protuberances seen in Figure 2.8A-D above.

Since the recipe for synthetic PRB coal char was modified, it was of high pertinence that X-ray diffraction spectroscopy can see if the functional groups of the new syn char simulate correspond with the actual PRB char, with an aim towards increasing the sp2- 


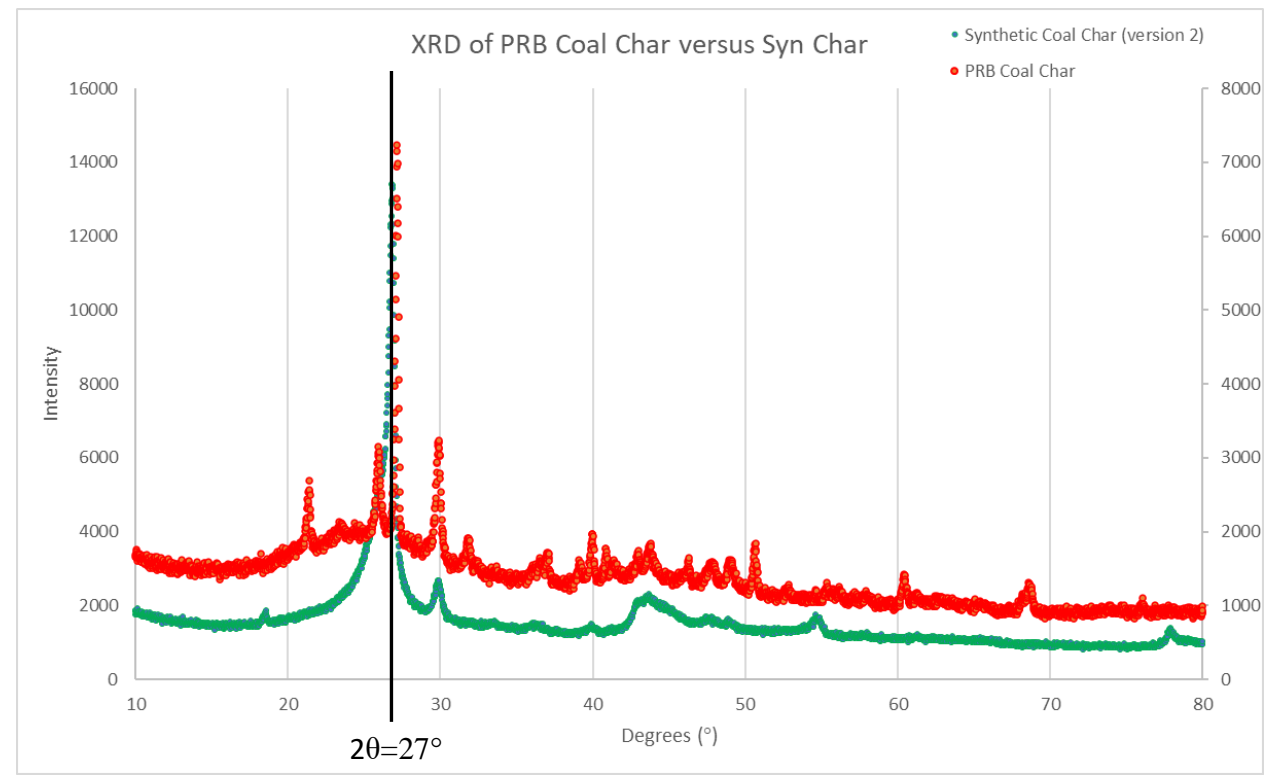

Figure 2.9 X-ray diffraction spectra of the Powder River Basin coal char (red) and Synthetic Powder River Basin coal char (green). The peak centered around 27-28 illustrates the strong presence of pyrolyzed graphitic carbon species.

hybridized carbon (graphite) to match the original sample of coal char. The resultant XRD (Figure 2.9) shows the excellent convergence of real PRB coal char and the simulated product, with prominent sp2-hybridized carbon peak located near $27^{\circ}$ which coincides with the increase in graphite content for the second rendition of the formula. Also noted that the SEM image (Figure 2.10) illustrates the protuberances seen in prior PRB coal char images (optical). It has been speculated that this will further reduce the conductivity to magnitudes seen in the literature previously noted and akin to the actual PRB coal char's DC electrical conductivity values due to percolation theory principles arising from these repeated measurements and simulations (see section: "Analysis of Compression on the Electrical Conductivity of Synthetic Coal Char" for further examination of annealing and compressive effects in conductivity). 


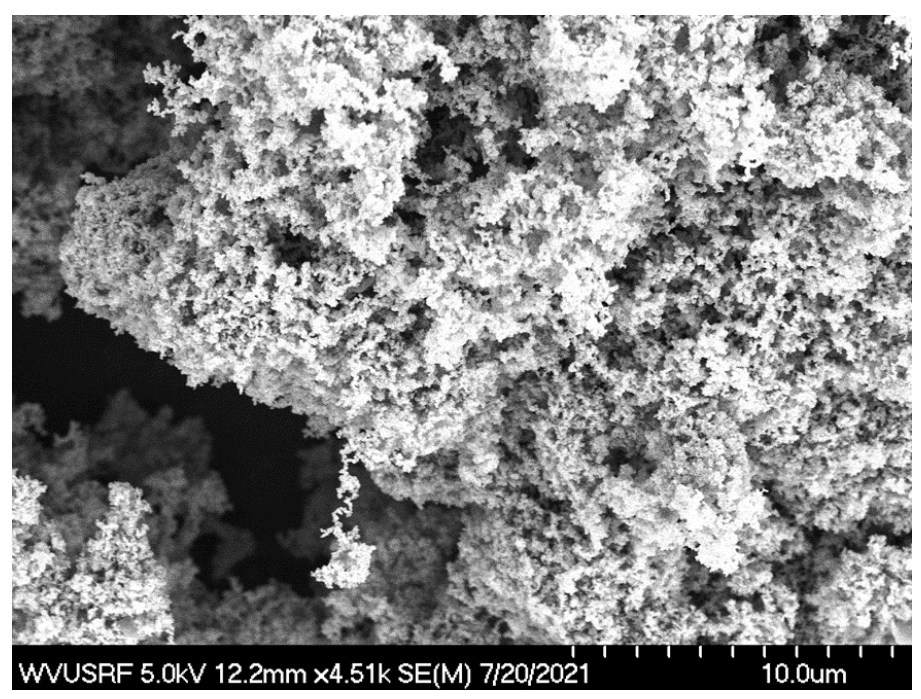

Figure 2.10 Scanning electron microscopy of the second version of Synthetic Powder River Basin coal char synthesized with the addition of more graphite species and increased homogeneity of ash coating using spin-drying methods as opposed to simple mechanically-mixing the original PRB simulate formula with $15 \%$ graphite species.

\section{Implementation and Experimental Verification of Dielectric Properties of $\mathrm{Fe}_{3} \mathrm{O}_{4}$ Nanoflakes in Micro-Reactor Using COMSOL}

To create modular reactors at the microscale and to facilitate creation of catalytic processes that far exceeds current microwave reactor coal conversion technologies, verification upon the work put forth by Yang et al. (2016) [38] and Zhao et al. (2018) [1] was attempted by simulating the experimental portion in a virtual framework and to toggle the conformation of the nanoflakes to match both literature and reality using COMSOL. An important value to optimize are return losses (RL) values. The return loss values are analogous to what reflection coefficients are to scattering parameters; put simply, they measure the power loss at the discontinuities during transmission of waves. In the literature, Yang et al. establishes that optimal nanodisc sizes are smaller than 10 microns (the group currently have synthesized flakes that fit this regime) with RL values of $-10 \mathrm{~dB}$ or less, establishes that $90 \%$ of incident EM-radiation is absorbed by the nanodisc and use to catalyze the reactor. Smaller discs allow for less anisotropy and therefore lower electric permittivity and better impedance matching 
(tying into the aspect of keeping reflection losses as minimal as possible). Both Yang et al. and Zhao et al. referenced obtaining return losses about $-30 \mathrm{~dB}$ to $-40 \mathrm{~dB}$. The RLs change based upon which portion of the $\mathrm{X}$ and $\mathrm{C}$-band of microwaves utilized in the experiment as return losses then decrease at greater frequencies towards $12 \mathrm{GHz}$ and beyond. In this study's experimental focus, we are also toggling frequencies in this area but are primarily focused on $2.45 \mathrm{GHz}$ and $5.85 \mathrm{GHz}$ (the resonant frequency of the magnetite nanoflakes).

Figure 2.11 below illustrates the precision of the COMSOL model as the RL ranges anywhere from $-70 \mathrm{~dB}$ to a more efficient $-40 \mathrm{~dB}$ loss. The return losses are substantially influenced by the meshing of the discrete element pieces that compose the micro-reactor; mesh refinement can aid in elucidating the actual solutions to Maxwell's equation at the microscale regime at the sharper edges and points in the DEM structure. In the current study and analysis, efforts to effectively modulate this with added temperature and the synthetic PRB coal char added in the nanodiscs array to simulate real-life, practical conditions to maximize yield.

\section{Simulation of $\mathrm{Fe}_{3} \mathrm{O}_{4}$ Nanoflakes in Micro-Reactor on Synthetic Coal Char bed}

In order to augment the electric and magnetic responses of synthetic coal char, we posit the addition of nanoparticles would enhance the overall effectiveness of absorbing electromagnetic radiation and reducing overall lost energy potential. There exists previous research into using shaped, ferromagnetic nanoparticles for usage in absorbing electromagnetic energy. These articles mainly focused on correlating Snoek's relation as a function of geometry of "soft" ferromagnetic targets, and thereby enhancing their magnetic susceptibility. This involves a considerable trade-off between skin depth, anisotropy, aspect ratio, and characteristic length. [39] Maximizing the magnetic susceptibility of nanoparticles (nanoflakes, nanorods, etc.), is pertinent to optimizing energy transfer from particle to environs with less wasted potential energy. Therefore, it is necessary to explore this phenomena further with sampling different types of ferromagnetic particles, created from previous hydrothermal 


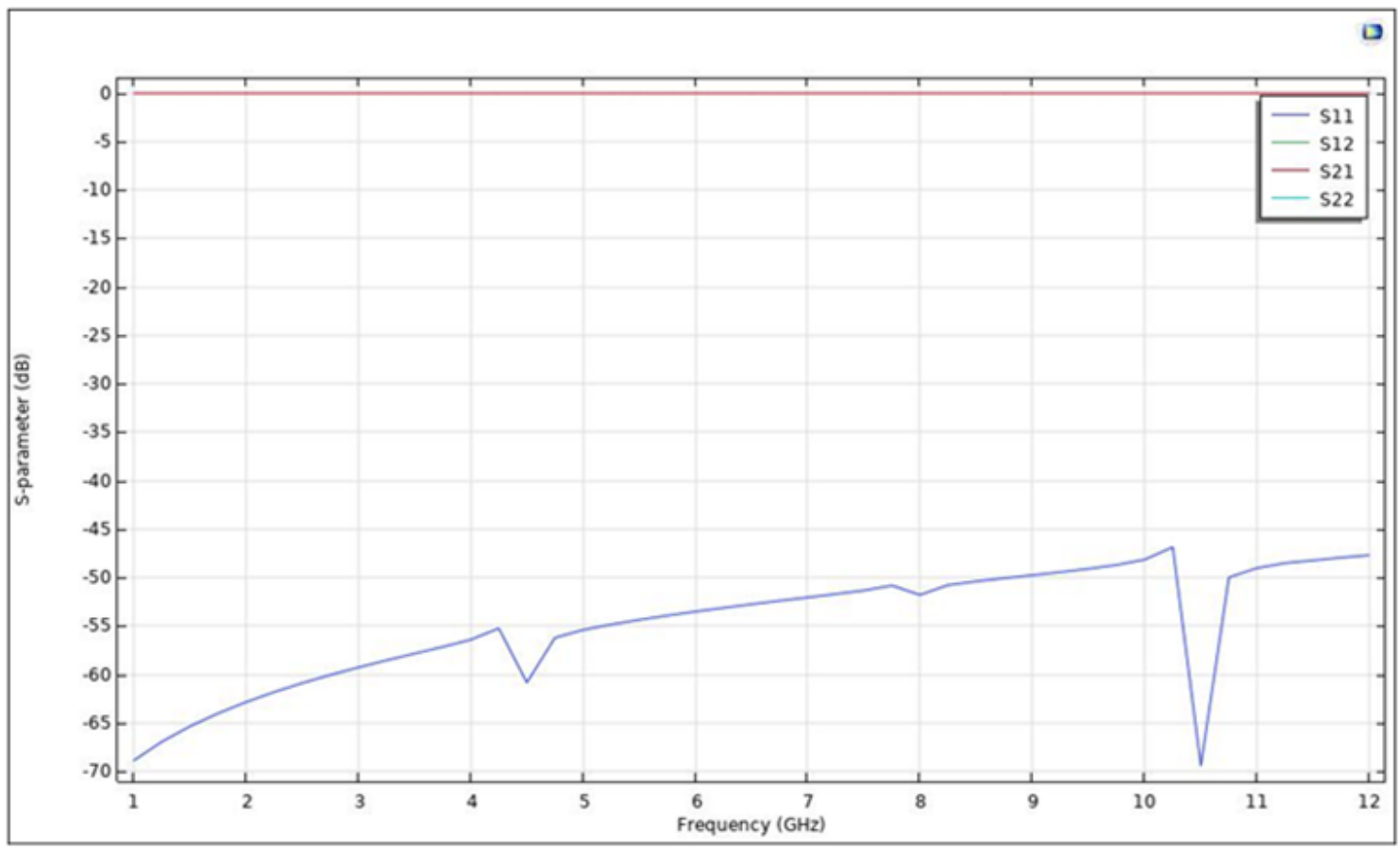

Figure 2.11 Scattering parameter results of COMSOL simulation of a mono-flake of magnetite $\left(\mathrm{Fe}_{3} \mathrm{O}_{4}\right)$ suspended in Nitrogen gas $\left(\mathrm{N}_{2}\right)$. It is important to note that $\mathrm{S} 11$ is equal to the total reflective power because $\mathrm{S} 21$ (transmission) is zero. The return losses match what Yang and Zhao et al. have tested previously in their respective groups.

synthesis. [40] To ensure adequate production of loss due to electrical and magnetic field interactions with the synthetic coal char, we need to optimize the the RL values of mono-flakes of $\mathrm{Fe}_{3} \mathrm{O}_{4}$ (magnetite) at varying microwave frequencies. A COMSOL simulation involving a facsimile of magnetite mono-flakes synthesized in real-life via solvothermal methods was performed in a Nitrogen gas atmosphere to emulate what Yang and Zhao et al. have tested in their research on RL values of shaped ferromagnetic particles. This led to Figure 2.11 and the scattering parameter results of said COMSOL simulation. Remarkably, the S-parameters matched with high fidelity and $-60 \mathrm{~dB}$ return loss at the targeted frequency of $2.45 \mathrm{GHz}$. Incorporating these mono-flakes would augment the heat generation of the synthetic PRB and thereby enhance catalytic efficiency for syngas formation reaction pathways.

Single particle simulations in COMSOL detailing a single magnetite nanoflake on a bed of synthetic PRB coal char substrate with $\mathrm{N}_{2}$ atmosphere have shown explicit depen- 


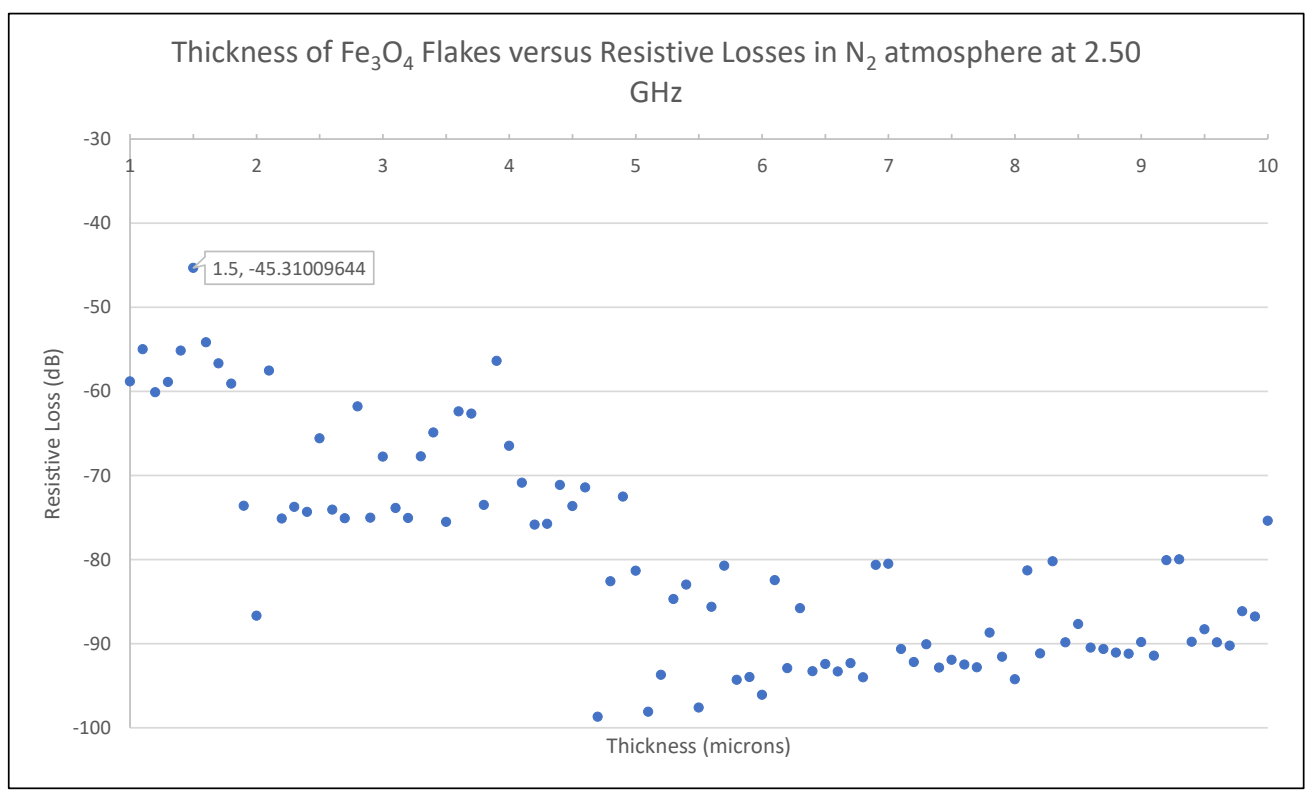

Figure 2.12 Scatter plot of magnetite nanodisc (flake) versus thickness in microns at the target frequency $2.50 \mathrm{GHz}$ created from a parameter sweep in COMSOL. The optimal thickness appears to be at 1.5 micron thickness with return losses at $-43 \mathrm{~dB}$.

dence on the absorption of microwave energy with the particle's thickness. The results in subsection $\mathrm{E}$ is annexed by attempting to determine whether the optimal thickness from previous COMSOL simulations could be improved with this duplicated magnetite flakes from our group. It can be shown that as the thickness of the particle decreases, the return losses decrease in a pseudo-linear fashion. The correlation could be readily seen in Figure 2.12.

The experimental implementation of the magnetite nanodiscs was initiated by a fellow graduate master's student, with his work ultimately replicating Zhao et al.(2018) [1] with similar results. The thickness of the magnetite flakes were slightly thinner than Zhao and group's attempt with a thickness of roughly 700 nanometers (0.7 microns), improving upon the several micron thickness reported by Zhao et al. Figure 2.12 illustrates the end result of the attempt of duplication from previous literature studies following similar synthesis and reproduced the magnetite flakes with high fidelity, with thinner flake monostructures that necessitates better conversion of microwave energy. Further analysis indicated this to be true with a single particle simulation in COMSOL detailing a single magnetite nanoflake on 


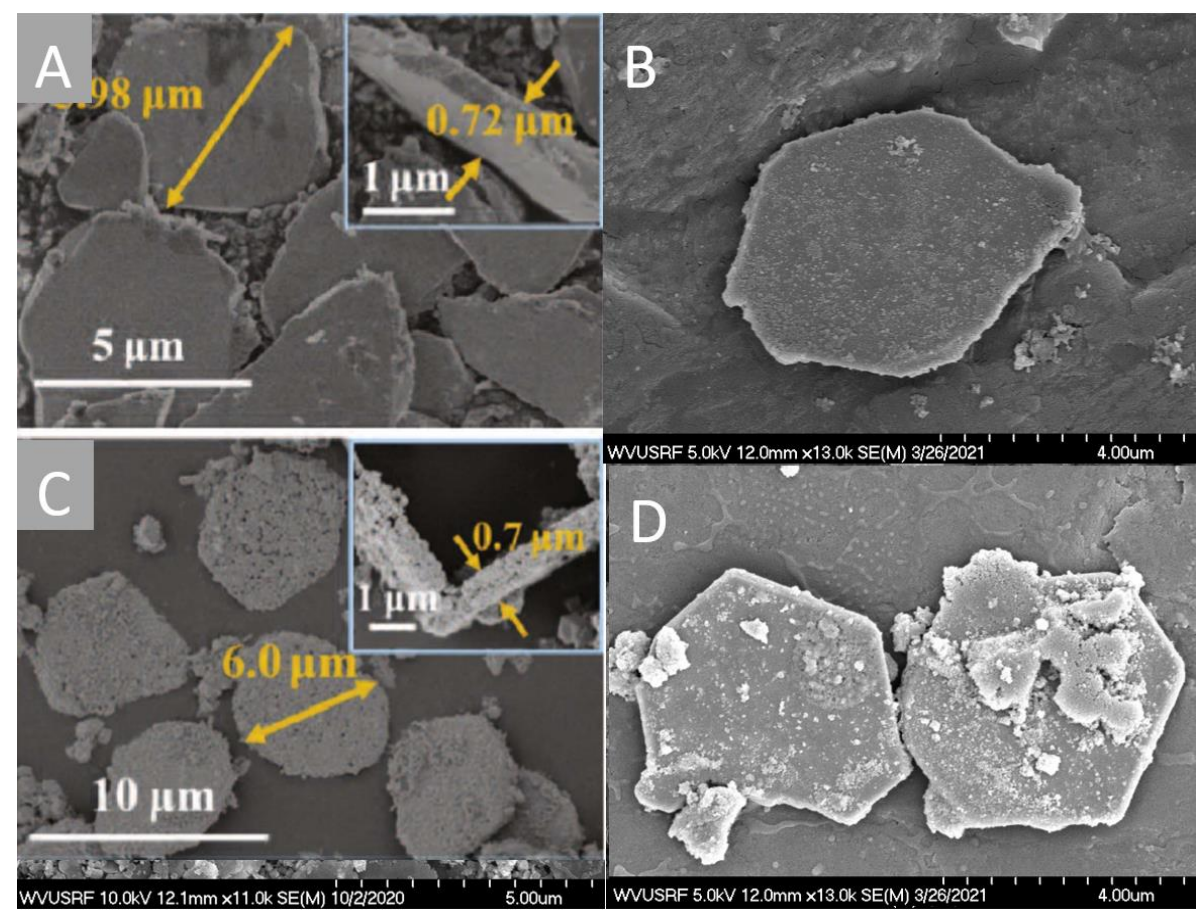

Figure 2.13 SEM imaging of $\mathrm{Fe}_{2} \mathrm{O}_{3}$ (subfigure A) and $\mathrm{Fe}_{3} \mathrm{O}_{4}$ (subfigure C) micro-platelet formations from literature (Zhao et al. 2018) [1] vs. Samples of $\mathrm{Fe}_{2} \mathrm{O}_{3}$ (subfigure B) and $\mathrm{Fe}_{3} \mathrm{O}_{4}$ (subfigure D) micro-platelet formation performed by the experimental portion of the group

a bed of synthetic PRB coal char substrate with $\mathrm{N}_{2}$ atmosphere with highly refined meshing and adaptive mesh control to obtain RL values of $-5.264 \mathrm{~dB}$ for 1.5 micron thickness and $-5.198 \mathrm{~dB}$ for 0.7 micron thickness. RL values of less than $-10 \mathrm{~dB}$ indicate higher than 90\% microwave absorption and vastly improves the losses from Yang's and Zhao's groups' previous attempts.

\section{Analysis of Compression on the Electrical Conductivity of Synthetic Coal Char}

There are numerous studies indicating the overpowering influence of compression and volume on physiochemical properties of materials. These studies show that compression can aid in creating preferred structural conformations due to shear strain and thereby increases overall strength of materials [41]. It is interesting to note the current research trends on 
mechanochemical properties can be modified to increase overall reactivity of the material for better coal conversion [42] without entertaining the priority of energy-intensive processes. This mechanochemical properties, therefore, can be assumed to modulate the electrical conductivity of materials under varying shear strain (read - compression in DC probes) with a mathematical basis in percolation theory and creating micro-channels that facilitate electrochemical processes [43]. The effective conductivity from section $\mathrm{C}$ of this paper illustrates that the electrical conductivity can vary wildly based upon various levels of shear strain impelled upon the compression dye used in a 4-point DC probe. With the more recent formulation of the synthetic PRB coal char (dubbed version 2 in the XRD in Figure 2.9), the addition of more graphite carbon to the mixture presents an issue with trying to overall reduce the conductivity to levels seen by [37] and our own sample of PRB coal char. The new conductivity values have increased substantially, which makes sense given that sp2hybridized carbon species has an exceedingly significant contribution to overall electrical conductivity and that the weight fraction of the graphite increased from $15 \%$ to $30 \%$. The conductivity values of the rectified syn char formula yielded $772 \mathrm{~S} / \mathrm{m}$ and a lesser-compressed trial of the same sample of syn char yielded $741 \mathrm{~S} / \mathrm{m}$ which is marginally less conductive.

After a satisfactory approach of coating the synthetic coal char to create synthetic coal char version 2, further improvements were employed to coat it even more uniformly and introduce those large granular ash material seen on the native dried PRB coal. This version was named "version 3" in the post-experimental discourse and throughout the rest of this body. Version 3 is noted to have $467.7 \mathrm{~S} / \mathrm{m}$ electrical conductivity, splitting the conductivity of the syn char version 2 by approximately a third - and approaching the $15 \%$ weight fraction of graphite version of synthetic PRB which has values of $370.4 \mathrm{~S} / \mathrm{m}$ for particles in the median bin of size 200 to 300 microns in radius (version 1). This further reinforces the theory that the electrical conductive network is impeded when a ash layer of metallic oxides and silica is present in a pyrolyzed sample of coal char. The optical images of this newer version can be seen in Figure 2.14, which when compared to the original coal 

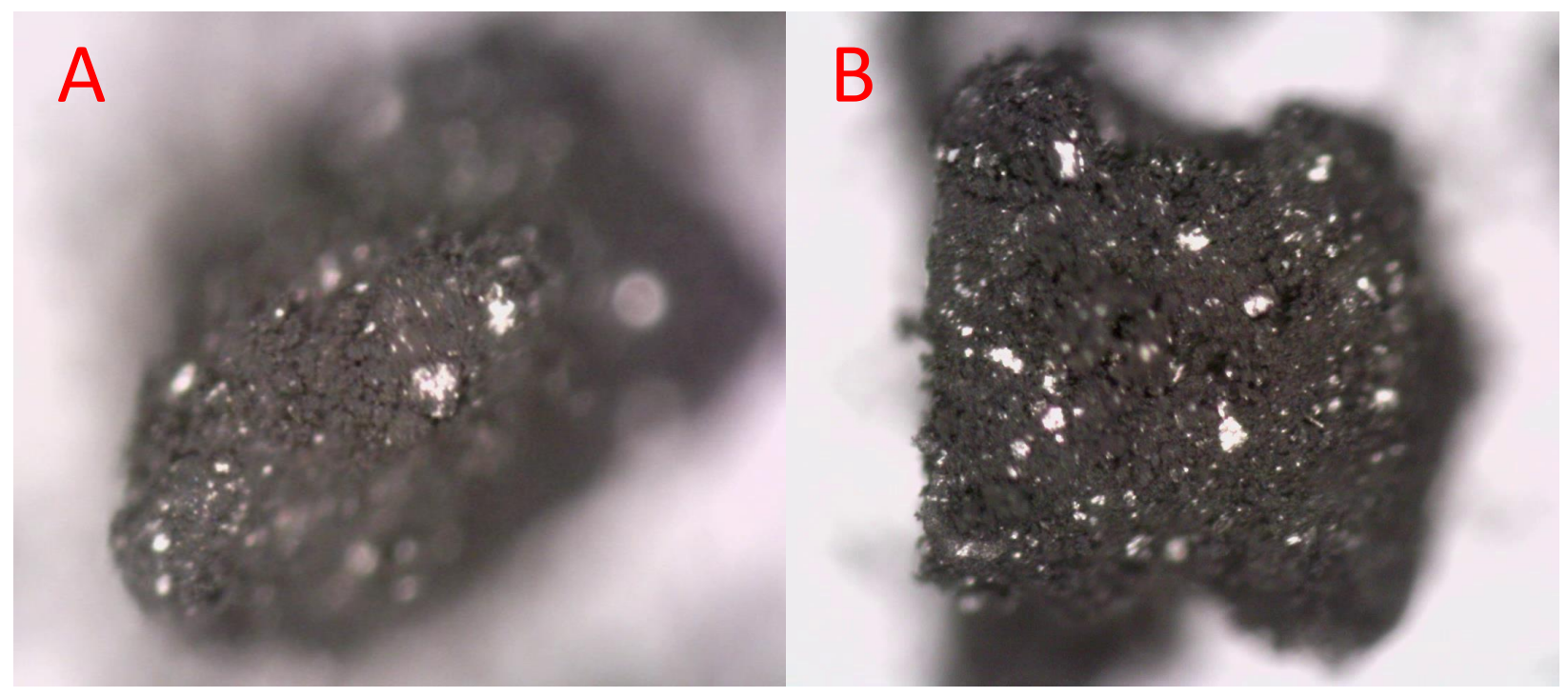

Figure 2.14 Images of Synthetic Coal Char (version 3) - spin-coated in ash to match XRD of the actual PRB coal char. Note the increased inclusion growth of fine ash matter (protuberances) on the surface of the synthetic coal particle in both subfigures, perfectly mimicking the actual PRB coal char images.

specimen, simulated the real sample with high precision and accuracy. The IV-curve results of these trials were consummated in the included Figure 2.15.

\section{Analysis of Electrical Conductivity of Pyrolyzed PRB Coal}

After repeated attempts to rectify the relatively non-conductive Powder River Basin coal char with the extremely conductive synthetic PRB coal char, it was determined that the original sample had a significant amount of oxide layers due to previously being torrified in air, at a temperature at or below 500 centigrade. It was then hypothesized that the 7 or 8 magnitude discrepancy could be due to the pyrolysis process of the actual PRB coal char and not the formula creating the synthetic PRB. All other preliminary tests shows the high fidelity of replication of the original sample of coal char and the synthetic coal char was pyrolyzed at a much higher temperature in an inert atmosphere. Therefore, it would behoove the study to emulate all pre- and post-processing of the syn char with respect to dried 


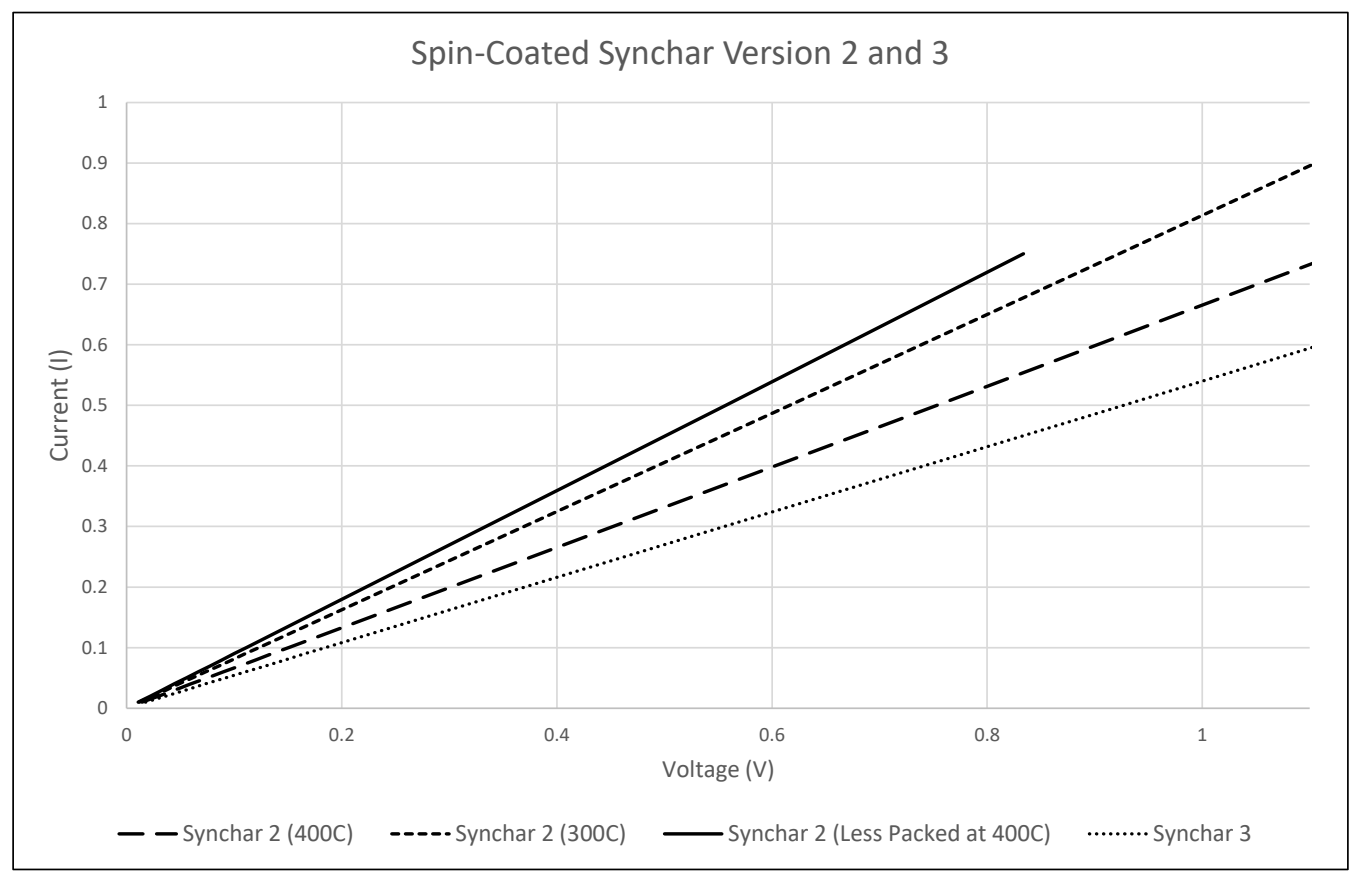

Figure 2.15 Current-voltage chart (also known as IV-curve) of the various trials of the synthetic PRB (version 2) with different pyrolyzed temperatures. Note the further decrease in conductivity in synthetic PRB (version 3).

PRB coal. Figure 2.16 was the culminating results of this attempt. As previously stated, the progenitor sample of the Powder River Basin coal was pyrolyzed at a significantly lower temperature as the synthetic version. With respect to the literature basis, Yin's conductivity values demonstrated a range of conductivity on the order of 7 or 8 magnitudes, from as resistive as approximately $10^{-6} \mathrm{~S} / \mathrm{m}$, to as conductive as approximately $100 \mathrm{~S} / \mathrm{m}$. As noted previously, this PRB coal char yielded similar resistive properties at a level of approximately $10^{-5} \mathrm{~S} / \mathrm{m}$. The sample of PRB coal char was torrified at $500^{\circ} \mathrm{C}$ for an hour but Yin et al.'s work on coal-char films charred them by ramping to temperatures exceeding $900^{\circ} \mathrm{C}$. Providing the inspiration to pyrolyze at a much higher temperature was via Yin et al's work [37], as they noted that annealing temperatures paradoxically increased conductivity despite the overall increase of the film crack ratios that may ordinarily decrease conductivity networks. The dried coal char was then pyrolyzed at temperatures similar to Yin et al. in 


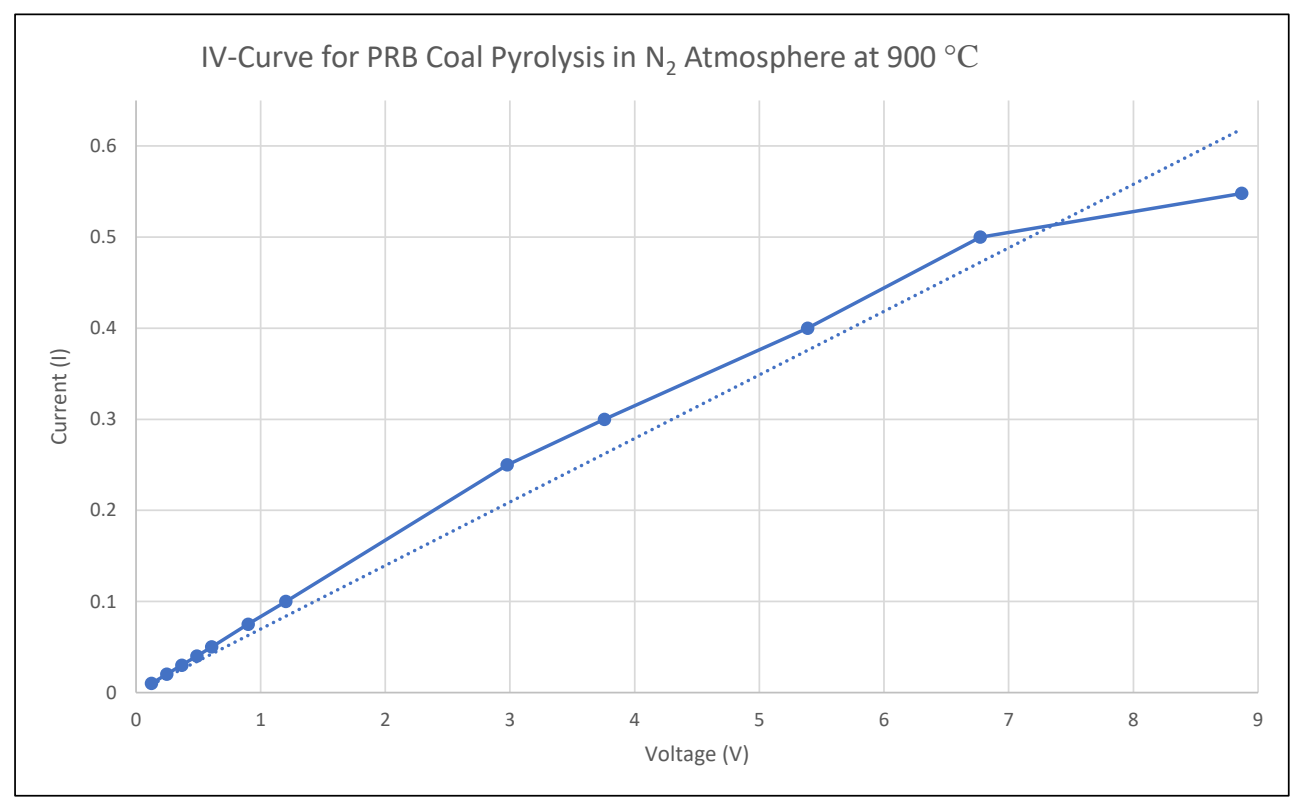

Figure 2.16 Current-voltage chart of pyrolyzed PRB coal char at temperatures at or beyond $900^{\circ} \mathrm{C}$. This resulted in a far greater conductivity than previous torrification at $500^{\circ} \mathrm{C}$, thereby verifying Yin et al.

an inert atmosphere environment (Nitrogen) at $900^{\circ} \mathrm{C}$ for roughly an hour to see if the conductivity also increases to such a degree.

Remarkably, it is shown in Figure 2.16 that the conductivity increased by several magnitudes, thereby validating Yin's analysis of coal-based film conductivity values and linking the major contribution of pyrolysis temperature and compression with the resultant conductivity. The inclusion of a highly conductive nanoparticles such as magnetite and the heightened conductive properties of synthetic coal char is presumed to be the primary reasons why the imaginary dielectric parameters were incongruent with realistic results.

\section{Simulation Results involving Thermal Power Generation of Shaped $\mathrm{Fe}_{3} \mathrm{O}_{4} \mathrm{Mi}$ - croparticles on Bed of Synthetic Char}

A complete facsimile of a microwave reactor was simulated in COMSOL to elucidate the total heat generation (measured in $\mathrm{W} / \mathrm{m}^{3}$ ), of the interaction of dielectric media with 
an electromagnetic wave set at the target frequency of $2.45 \mathrm{GHz}$ and using the power density of a WR-340 microwave reactor used at NETL operating at 2000 Watts. To test the hypothesis on the influence of particle morphology on dielectric losses for usage in microreactors, two basic shapes of the $\mathrm{Fe}_{3} \mathrm{O}_{4}$ microparticles were placed on a bed of synthetic PRB char. To keep the simulation testing electromagnetic loss at the most basic level and follow ab initio principles, the microwave energy was not pulsed, which deviates from real microwave reactors. However, in this circumstance, a few milliseconds of microwave energy suffices for preliminary study on EM losses. Several electrical conductivities were analyzed, ranging from $10^{-5} \mathrm{~S} / \mathrm{m}$ to the conductivity of the synthetic char version 3 at $495 \mathrm{~S} / \mathrm{m}$. It was determined through previous study that morphology may also play a key role in creating localized, enhanced power generation through edge effects; this led to this being a major factor in these simulations. Other variables tested included the angle of the electric field with respect to the plane of the reactor basin where the particle rests; the inclusion or absence of a magnetite particle; the thickness of the particles; the ports of the reactor to resolve absorption and reflectance of the incident electromagnetic waves; and finally, the diameter of the particle to see which variable dominates the total power generation. By setting one variable independent of the others, one could observe the most efficacious way of creating a reactor environment where the electric and magnetic field interactions are at a maximum, thereby maximizing loss and creating a thermal gradient.

One variable to test was the thickness of the particle and how it may affect the absorption of EM-radiation. It should be obvious that thicker particles would lead to higher absorption rate, but obtaining a homogenous synthesis of nanoparticles with the same dimensions can be prohibitively expensive and time-consuming and would be economically disadvantageous. Fortunately, the results concluded that the thickness of the particles shown little to no variance of total power generated via Figure 2.18. This should allow for some variance in following the synthetic procedures without affecting the overall performance of a hypothetical syn char and nanoparticle reactor. 


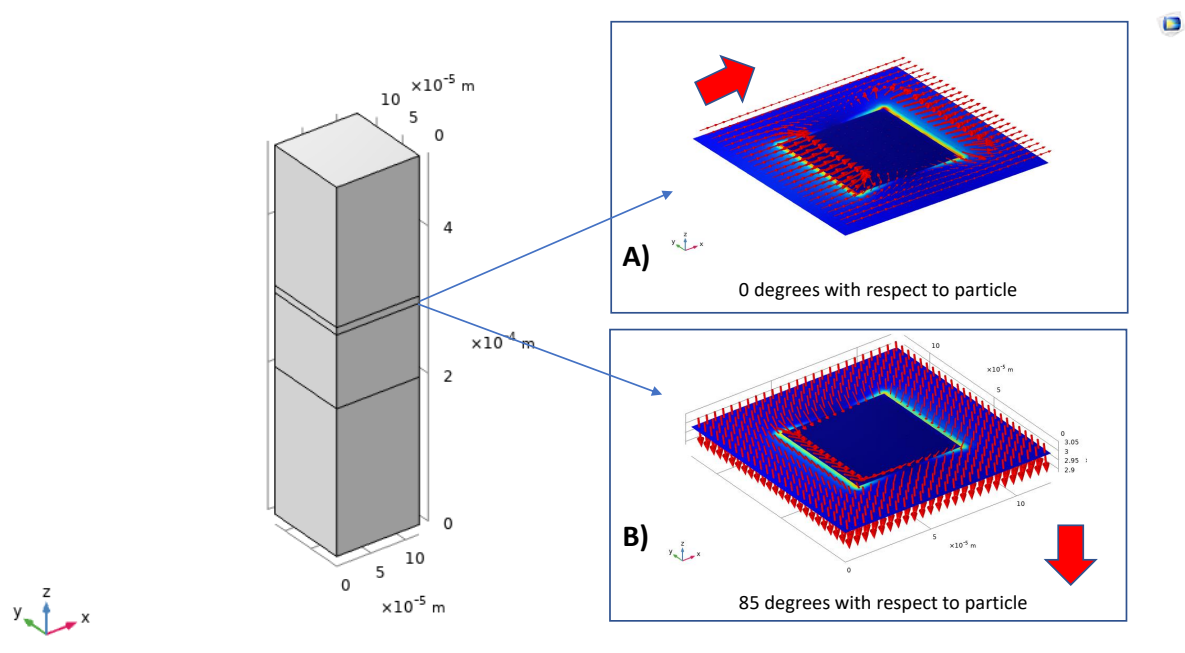

Figure 2.17 Helpful diagram illustrating the direction of the electric, and by extension, magnetic field across the reactor domain. Subfigure A is where the simulation sets the electric field at 0 degrees with respect to (w.r.t) the particle/reactor. Subfigure B is where the simulation aligns the electric field at 85 degrees normal to the plane of the reactor where the particles and syn char bed rest.

To better assist visualization of the electric and magnetic field lines permeating the domain of the reactor, Figure 2.27 shows the direction of the electric field (and thereby the B-field perpendicular to said electric field). 0 degrees with respect to the particle is seen on Subfigure A, Subfigure B shows 85 degree electric field vectors going from Port 1 (top) to Port 2 (bottom).

The largest disparities arose from changing electrical conductivity (shown in Figure 2.19), where the highest conductivity (495 S/m) shown some reflectance (Figure 2.19A) perhaps due to a heightened electrostatic interaction with the highly conductive reactor bed and reacts strongly as the angle with respect to the reactor basin nears 90 degrees (incident on particle and bed). It appears that the radius of 30-40 microns were the most consistent in generating the most power, but the effects on thickness and particle radius showed no productive correlation in terms of generation. The electrical conductivity set at $10 \mathrm{~S} / \mathrm{m}$ showed a magnitude better results in energy generation. The presentation of particles showed several magnitudes increase despite the relatively conductive synthetic carbon bed, further proving 


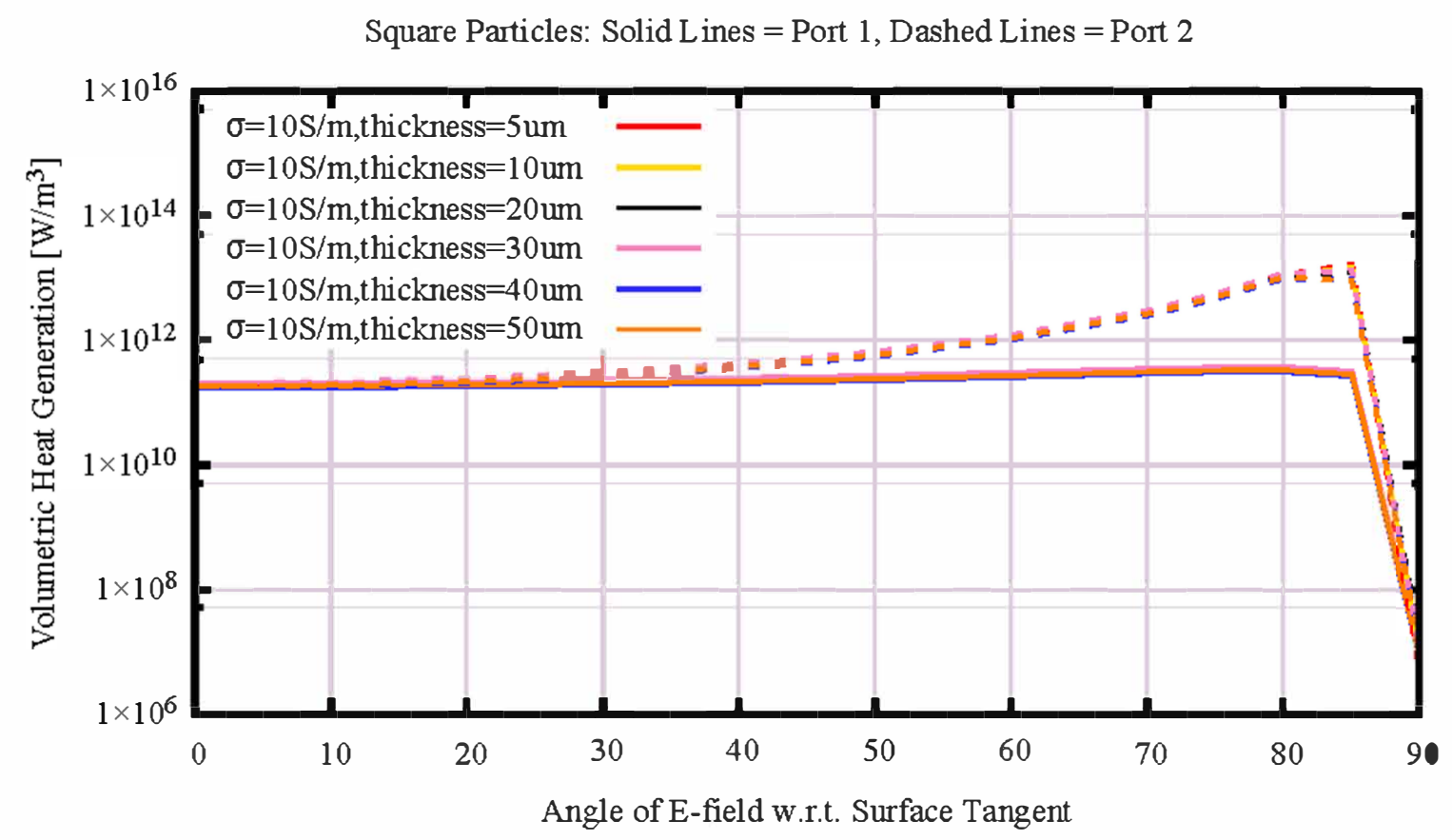

Figure 2.18 Comparison of thickness of square magnetite particles with power generation with a bed of syn char at $10 \mathrm{~S} / \mathrm{m}$. The changing of thickness negligibly affects power generation.

the multiplicative effects of a ferromagnetic on the order of magnetite listed in Figure 2.20 and Figure 2.21.

Another crucial comparison is the evidence of whether the presence of shaped particles enhances the local field immediately surrounding the particle. As such, the following comparison was made between the presence of a rectangular particle on a bed of syn char and the lack of said particle with just the reactor holding the synthetic PRB coal char, compiled in Figure 2.20. As clearly evident in the following figure, the mere addition of shaped ferromagnetic particles enhances the power generation (in $\mathrm{W} / \mathrm{m}^{3}$ ) due to EM-loss by several fold. Comparing the power generation in terms of color-map leads to a better visualization in how the particle influences local heating via geometry of the particle and reactor interface. Figure 2.21 achieves this and shows how the edge and vertices of the square prism (Figure 2.21B) enables said enhancement, whereas the lack of magnetite elicits no such 

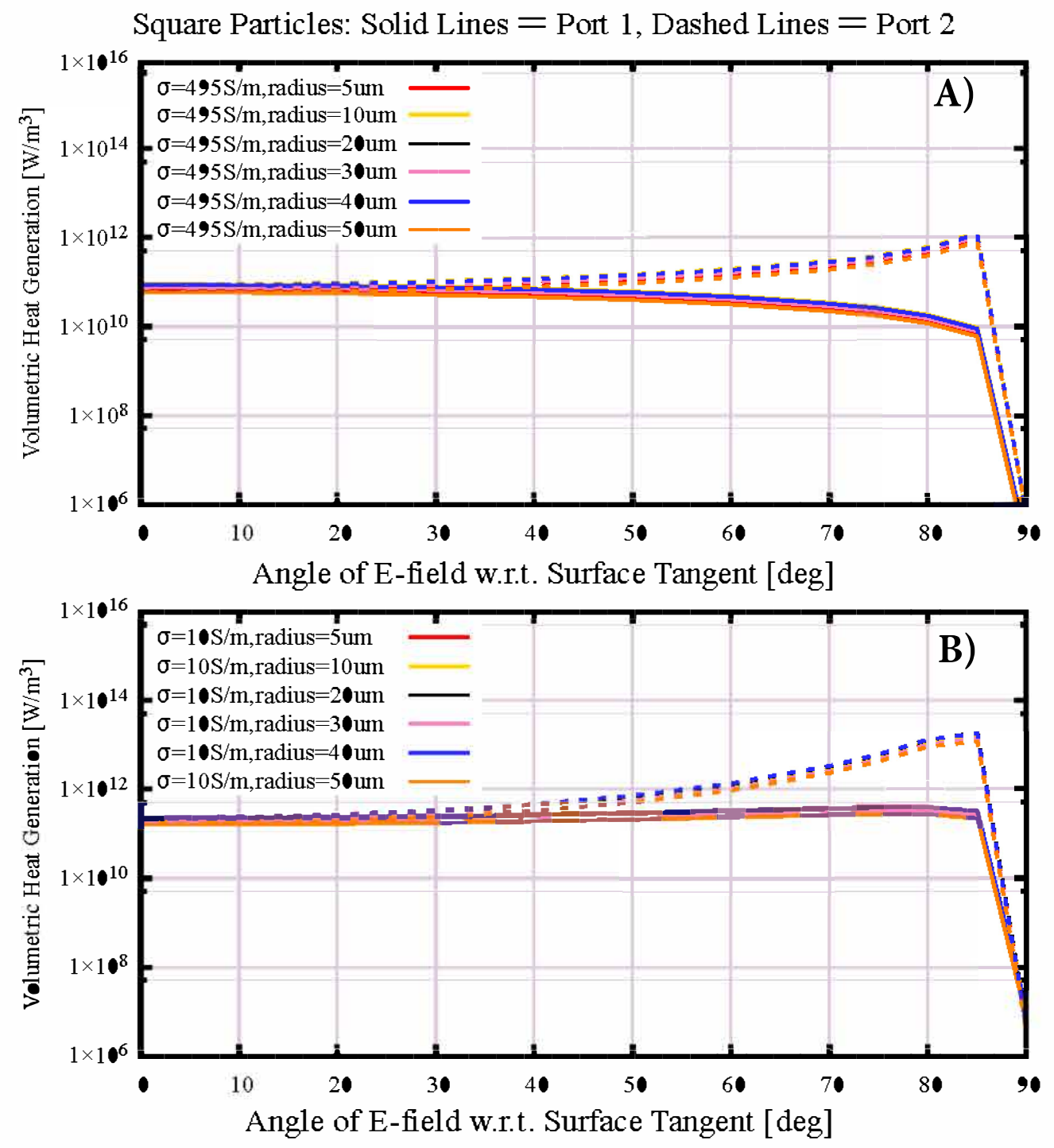

Figure 2.19 Comparison of electrical conductivity of square magnetite particles with power generation with a bed of syn char at $495 \mathrm{~S} / \mathrm{m}$ (syn char version 3 in subfigure A) and 10 $\mathrm{S} / \mathrm{m}$ (inert, pyrolzyed PRB coal char in subfigure B)

effect, shown via Figure 2.21A.

As detailed previously, two types of geometries, triangular and square-shaped particles, were simulated in COMSOL. In order to ascertain which type of geometry leads to the most optimal power generation, with particular interest in edges and vertices of said shapes. These particular shapes were chosen due to their relatively straightforward creation at the 

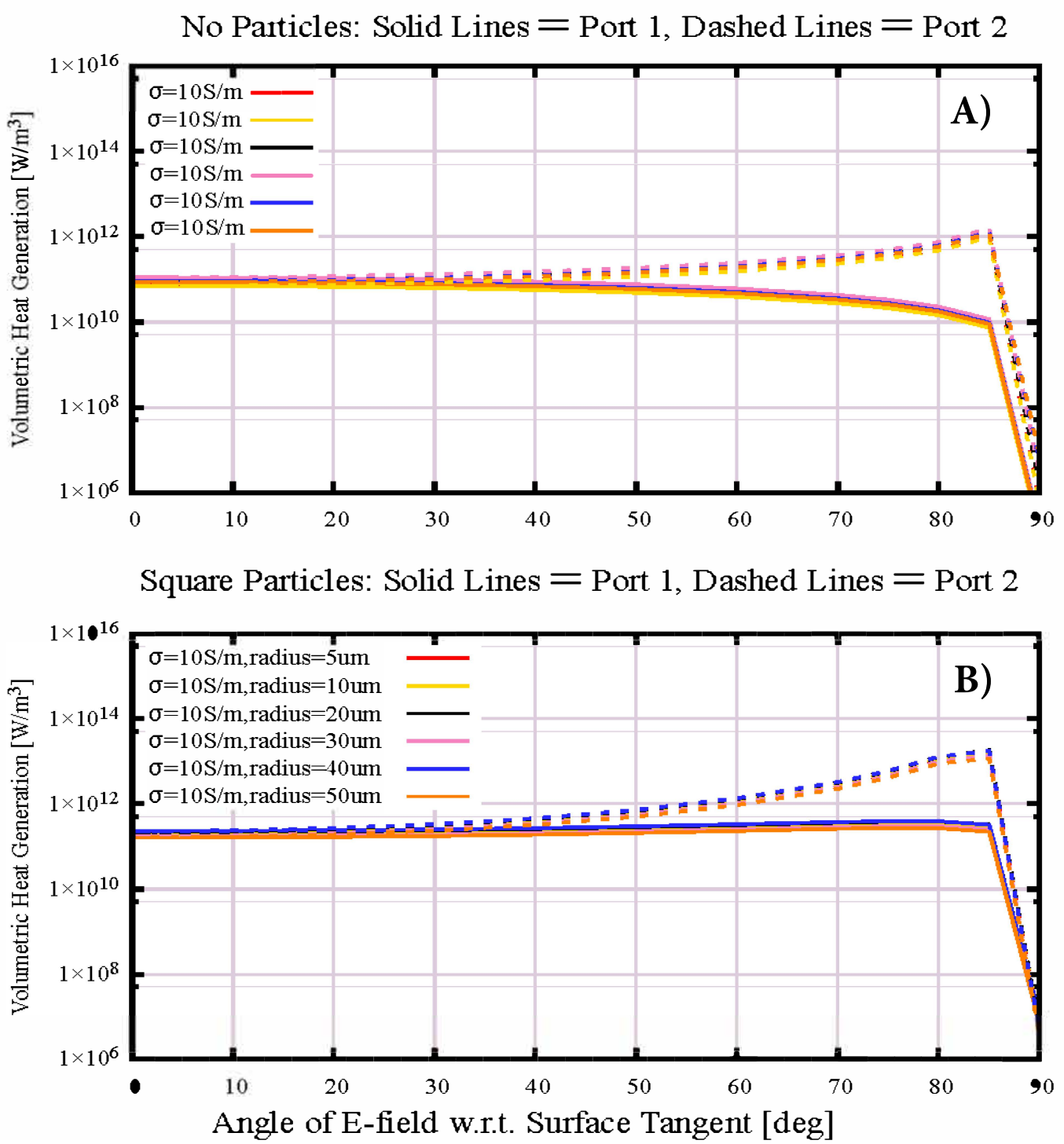

Figure 2.20 Comparison of power generation of just the bed of syn char (subfigure A) and the presence of square magnetite particles (subfigure B). The presence of particles multiplies the power generated by several fold.

microscopic level and the fact that these two shapes are the simplest n-gons (read: polygons) in existence. It is pertinent to keep these initial shapes as basic as possible to analyze the contribution of edges and vertices to electromagnetic losses. The most significant proportion of losses tends to be electrical losses, so a comparison of the electric field maximum between each particle at varying angles were set up. Figure 2.22 illustrates a stark contrast 


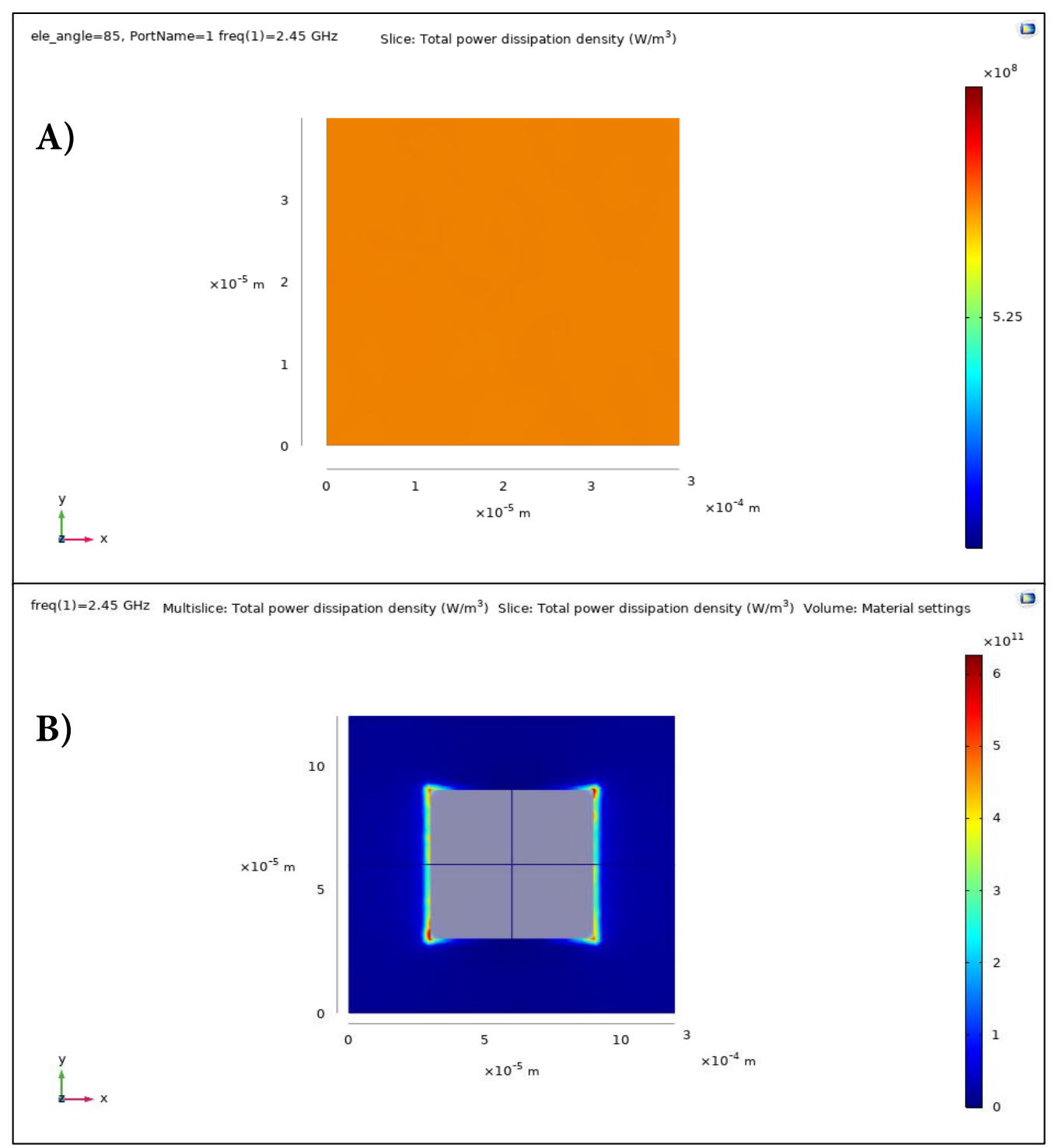

Figure 2.21 Comparison of power generation of just the bed of syn char (subfigure A) and the presence of square magnetite particles (subfigure B). Clearly depicted is the 3-4 magnitude increase in power density output.

between the electric field norm $(\mathrm{V} / \mathrm{m})$ of triangular and rectangular-shaped particles, with the quadrilateral particles vastly exceeding the electric field of the triangular particle by several magnitudes akin to the total power generation of square magnetite particles.

Figure 2.23 is the graphical representation of the difference between the two shapes. For the triangular-shaped particle (Figure 2.23A): the in-plane electric field distribution 
Triangle Particles: Solid Lines $=$ Port 1 , Dashed Lines $=$ Port 2
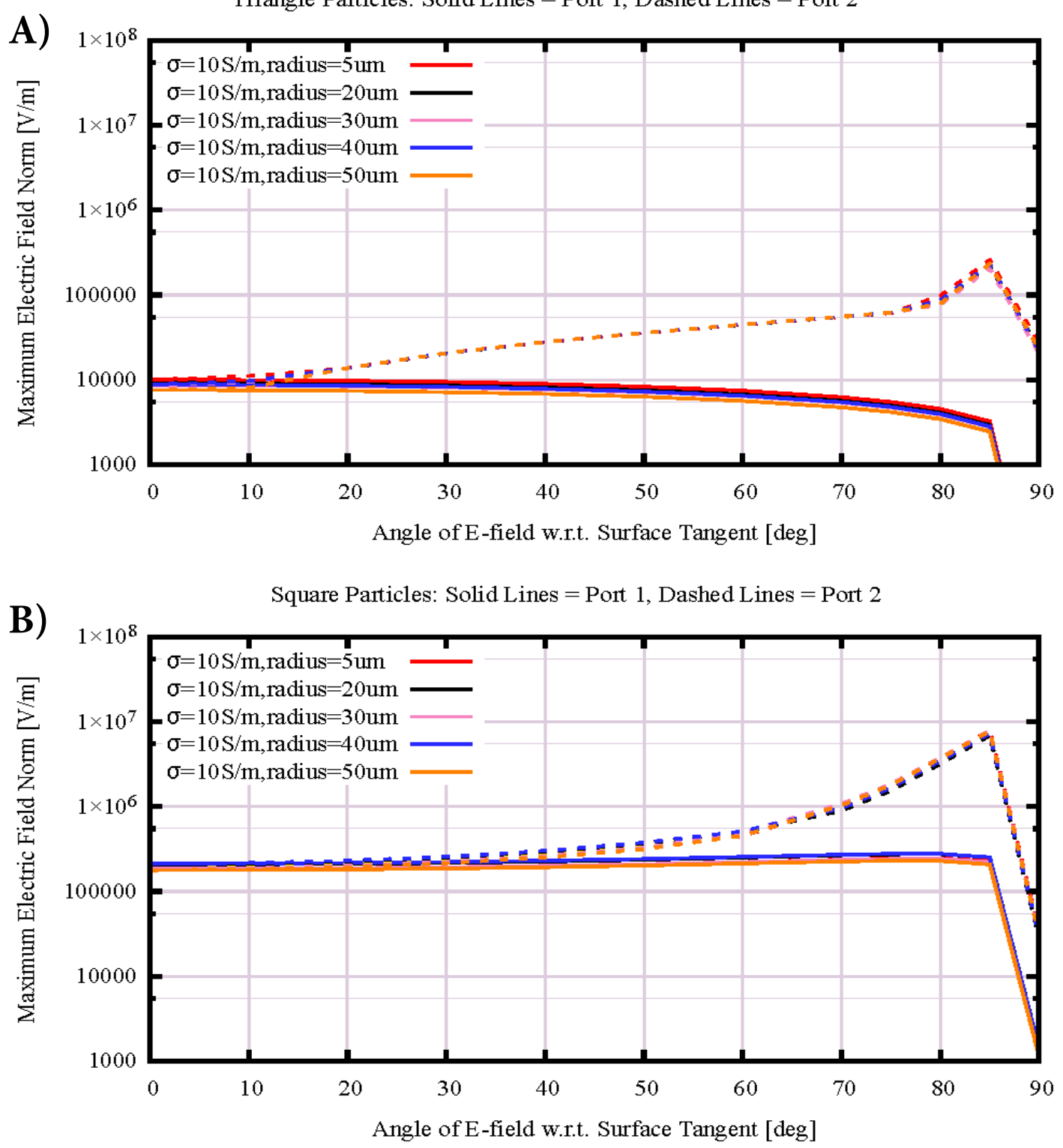

Figure 2.22 Comparison of the electric field norm $(\mathrm{V} / \mathrm{m}$ ) of triangular (subfigure A) and square particles (subfigure B). Similar to the power generation plots, the electric field for quadrilateral particles exceeds the triangular particles by several magnitudes.

clearly depicts the "focusing" action of the tip of the triangle with slight deviation of electric field. For the square-shaped particle (Figure 2.23B): the in-plane electric field distribution shows the rearranging field around the edges of the square particle, unequivocally detailing the interaction of the ferromagnetic particle with the incident microwave radiation. 


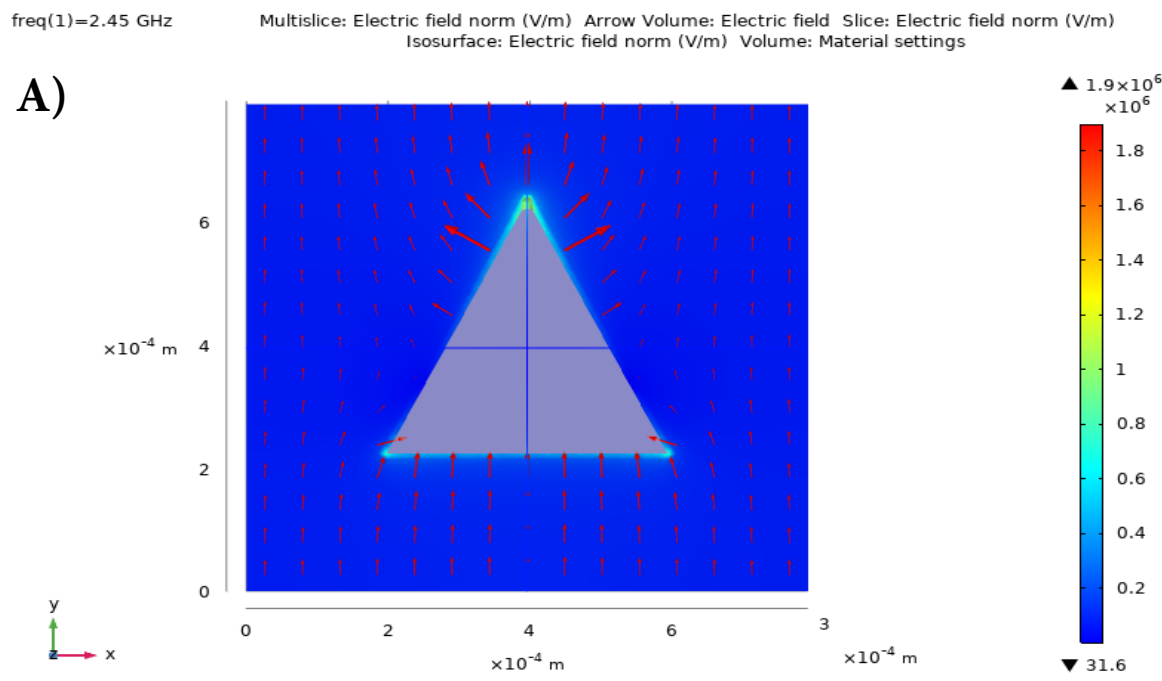

freq $(1)=2.45 \mathrm{GHz}$ Multislice: Electric field norm $(\mathrm{V} / \mathrm{m})$ Arrow Volume: Electric field Slice: Electric field norm $(\mathrm{V} / \mathrm{m})$ Volume: Material settings $\square$

B)
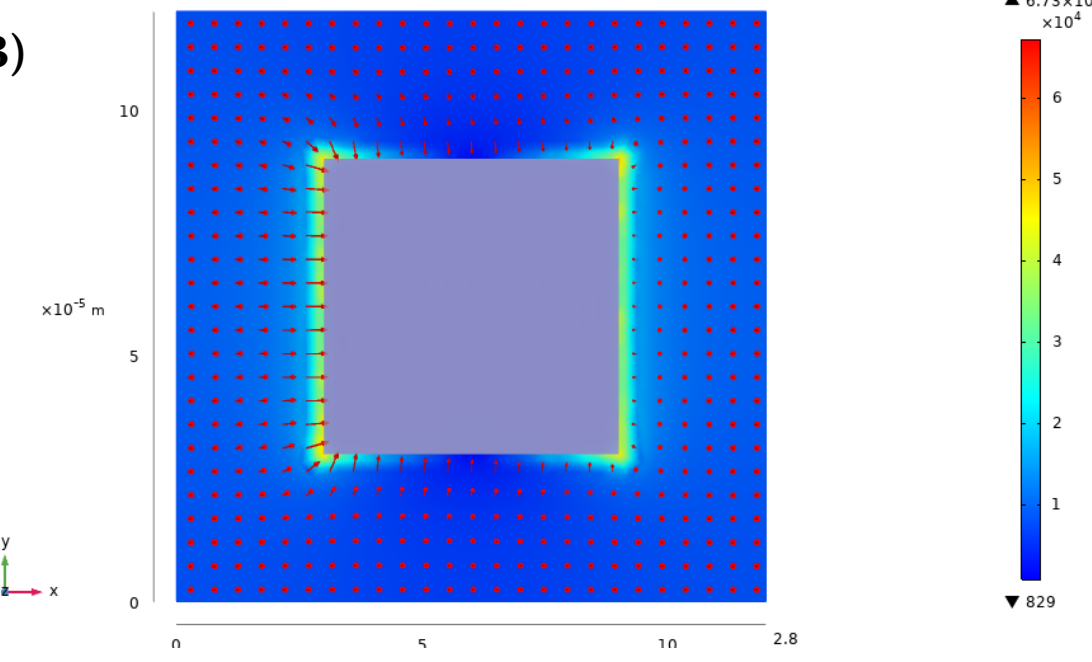

Figure 2.23 Comparison of the electric field norm $(\mathrm{V} / \mathrm{m}$ ) of triangular (subfigure A) and square particles (subfigure B). Electric field is toward the viewer in the xy-plane. Notice the electric field vectors and their interactions of the two shaped particles, showing the influence of shaped-particles on incident microwave radiation.

In order to completely describe the electromagnetic interaction with shaped particles on the bed of syn char, one must also investigate the local magnetic fields induced by the microwave waves and their field lines and strength, altered by the ferromagnetic magnetite's presence. Light, in the form of electromagnetic waves, propagates in a helical motion with both the component electric and magnetic flux field (the B-field) perpendicular to one an- 
other. As detailed in the prior schematic in Figure 2.27, outlining how the angle/orientation of the electric field is "swept" in the simulation study. Characterization of the energy flux of the EM-field can be utilized to see how shaped particles direct the flow of energy through the particle and substrate bed. This is achieved via the Poynting vector, which describes the energy transfer properties of the electromagnetic wave and is encapsulated in Figure 2.24.

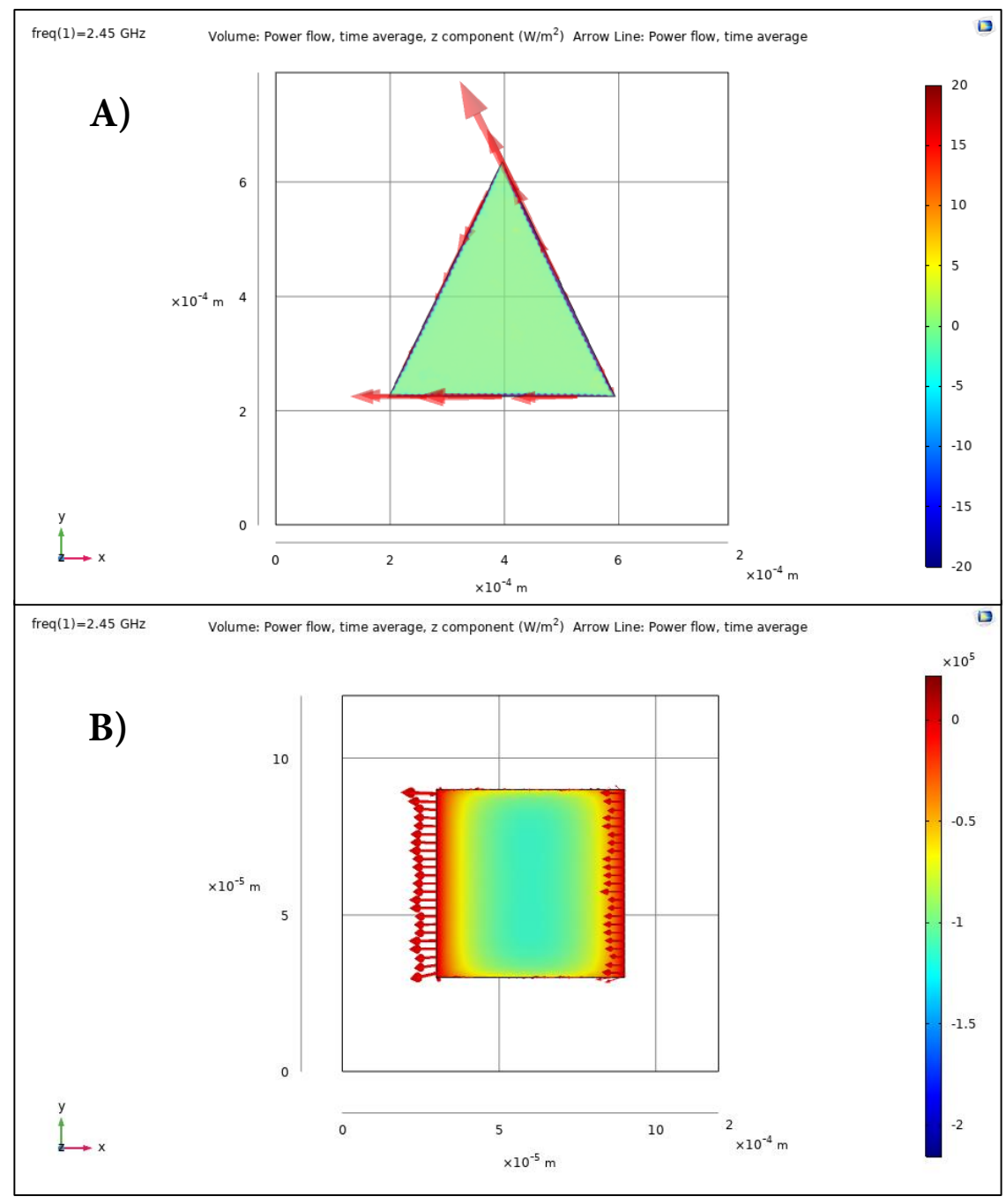

Figure 2.24 The norm of the Poynting vector that details how the energy of the electromagnetic waves propagate through media. Triangular particles (Subfigure A), shows that the Poynting vector norm pervades throughout the triangle particle with little difference of flux except for the periphery of the shape. Contrasted to that of the square particle (subfigure B), which shows the focusing of the electromagnetic energy away from the edges of the square prism. 
The Poynting vector is the directional energy flux of the electromagnetic field $\left(\mathrm{W} / \mathrm{m}^{2}\right)$ and is mathematically a continuity equation, which is used for conservation of energy laws. [44] Poynting vectors are a practical way to describe the electromagnetic transfer of energy across a coaxial line, the basis of vector network analysis in the microwave reactor simulation. This could be helpful in elucidating how the energy flux travels and orients itself around the particle-bed matrix for designing the most effective way to transfer dielectric energy for micro-scale reactors. Simulations in this study confirmed that the most efficient way of absorption of microwave energy is 85 degrees normal (directed onto the plane of the particle and reactor basin). Knowing this, the B-field must propagate perpendicular to this direction and thus results in the magnetic flux field permeating the prism in a manner seen in Figure 2.25, with field lines following the right-hand rule.
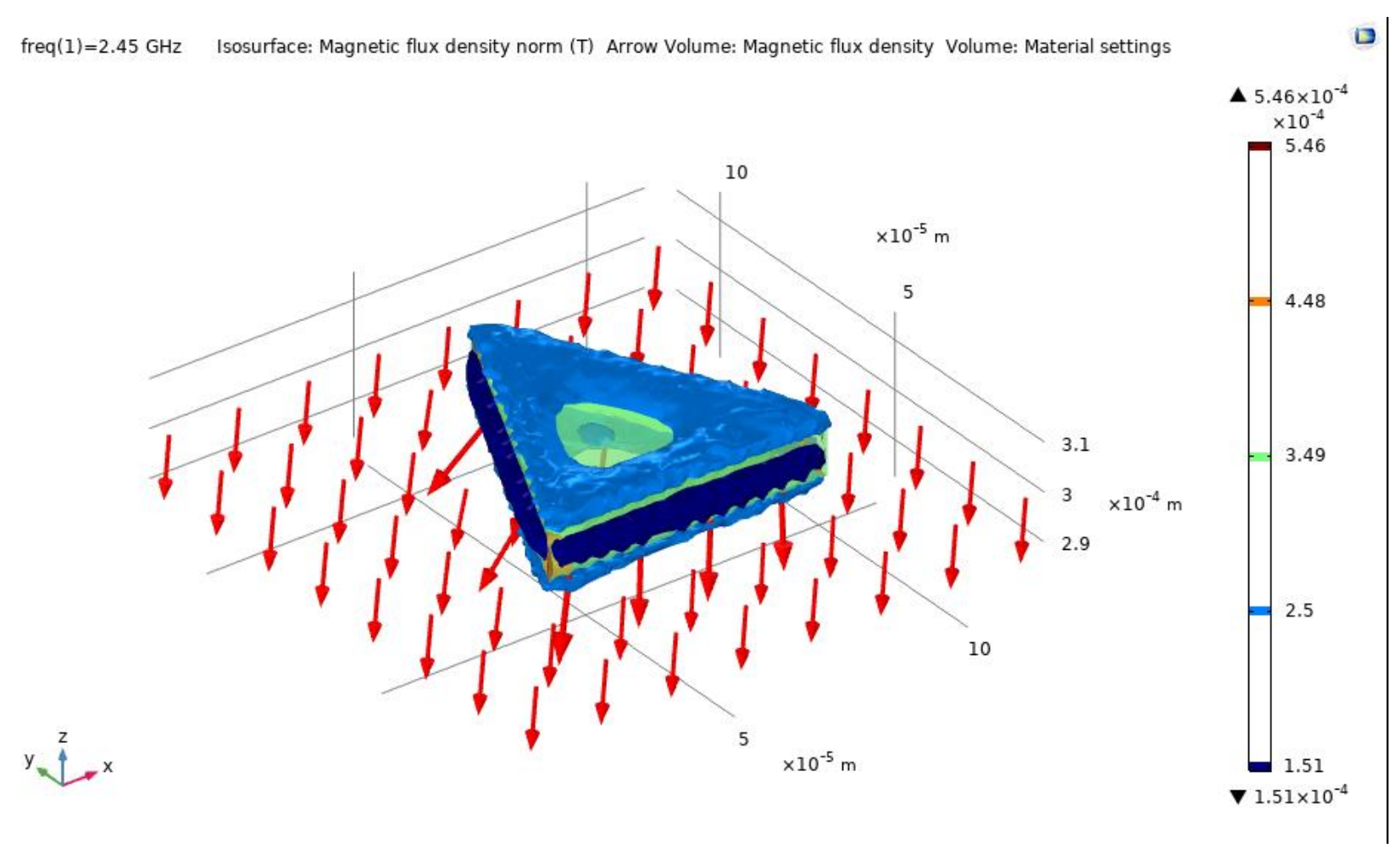

Figure 2.25 Isosurface/multislice representation of the magnetic flux density (B-field) with magnetic flux field vectors illustrating the edge effects of the triangular particle. The isosurface coupled with the arrow depiction of B-field vectors shows how the magnetic field lines concentrate in the triangular-shaped particle. 
Figure 2.25 is an isosurface and multislice representation of the magnetic flux density (B-field) with magnetic flux field vectors illustrating the edge effects of the triangular particle. The isosurface coupled with the arrow depiction of B-field vectors shows how the magnetic field lines concentrate in the triangular-shaped particle. Figure 2.26 is a volumetric representation of the magnetic flux density (B-field) of a square-shaped particle. The magnetic flux density clearly depicts the poles of the square prism, with enhancement of the magnetic flux norm along the y-axis of symmetry. The field strength is several orders larger than Earth's magnetic field, still significant enough to direct and enhance local heating. When comparing same area fraction (kept constant at 0.25) and sweeping corresponding length parameters and Port 1 and 2; square particles exceed total power dissipation by triangular-shaped prisms by several orders of magnitude, as seen in the graphical representation in Figure 2.28. The syn char bed was kept at a constant conductivity of $10 \mathrm{~S} / \mathrm{m}$ and swept at same angle and frequency.

The increased amount of power dispersion is presumably due to the presence of rectangular prisms containing more vertices and higher order of planar symmetry, leading to better emission of microwave energy via EM-loss. The visual depiction of the edge effects are seen via Figure 2.29A, with energy concentrated at the tip of the triangle and the energy concentrated around the periphery of the square-shaped particle (Figure 2.29B). Studying these energy dissipation models led questions on how to best choose a shaped particle for micro-reactor design. Triangular antennae in the form of a repeated array sequence using the triangle-shaped particles could be utilized to form a pseudo-electron beam gun in the form of a heating aperture.Future array simulations could be developed studying such a phenomenon. In the case of rectangular particles, the heating needed for the catalytic process requires an exogenous process that is more symmetric, i.e. uniform, in its ability to heat the surrounding area, albeit with lack of focusing of the dielectric energy form the losses. A coupling of these shapes (triangles and squares in tessellated arrays), could be another possible avenue of exploration in the near-future. 


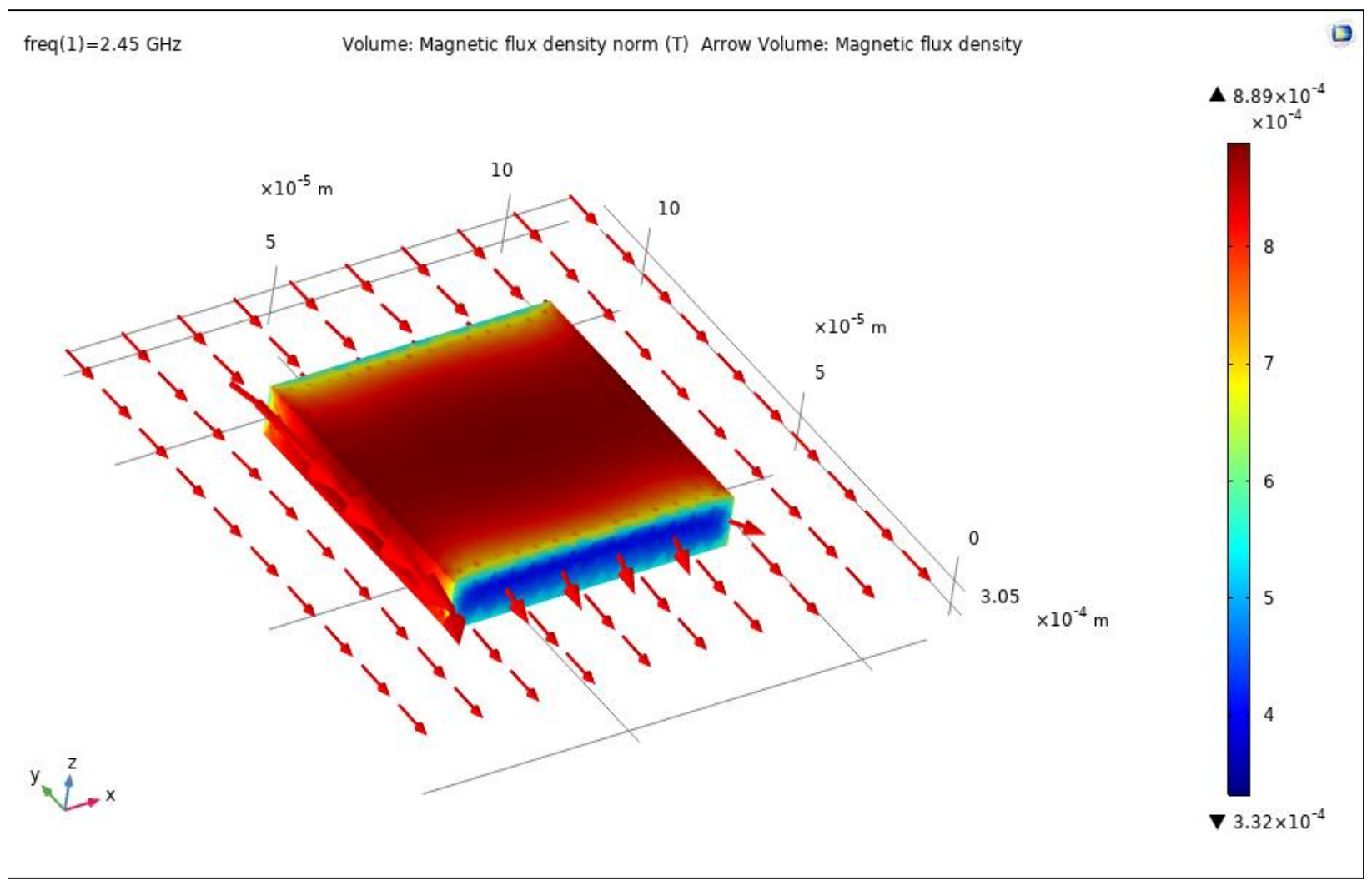

Figure 2.26 Volumetric representation of the magnetic flux density (B-field) of a squareshaped particle. Red arrows indicate direction of B-field. The magnetic flux density clearly depicts the poles of the square prism.

As discussed previously, the electrical (resistive) losses tends to be several orders of magnitude greater than magnetic loss (Figure 2.27A), and can clearly be seen via Figure $2.27 \mathrm{~B}$. The symmetry of where the edge enhancement occurs is orientation-dependent due to the right-hand rule of Maxwell's Equations and the Lorentz Force law and in this case is located on a y-axis of symmetry in Figure 2.27. Constructive interference of the electromagnetic radiation and the presence of magnetic polar effects appearing in the magnetic loss contour plot, creates a "focusing" effect indicative of higher-order dielectric losses on the edges of the square prism. These higher-order moments of the electromagnetic field and their interplay can presumably lead to much higher heating than a thermocouple sensor on the outside of the reactor could accurately describe. This result infers the existence of strong 


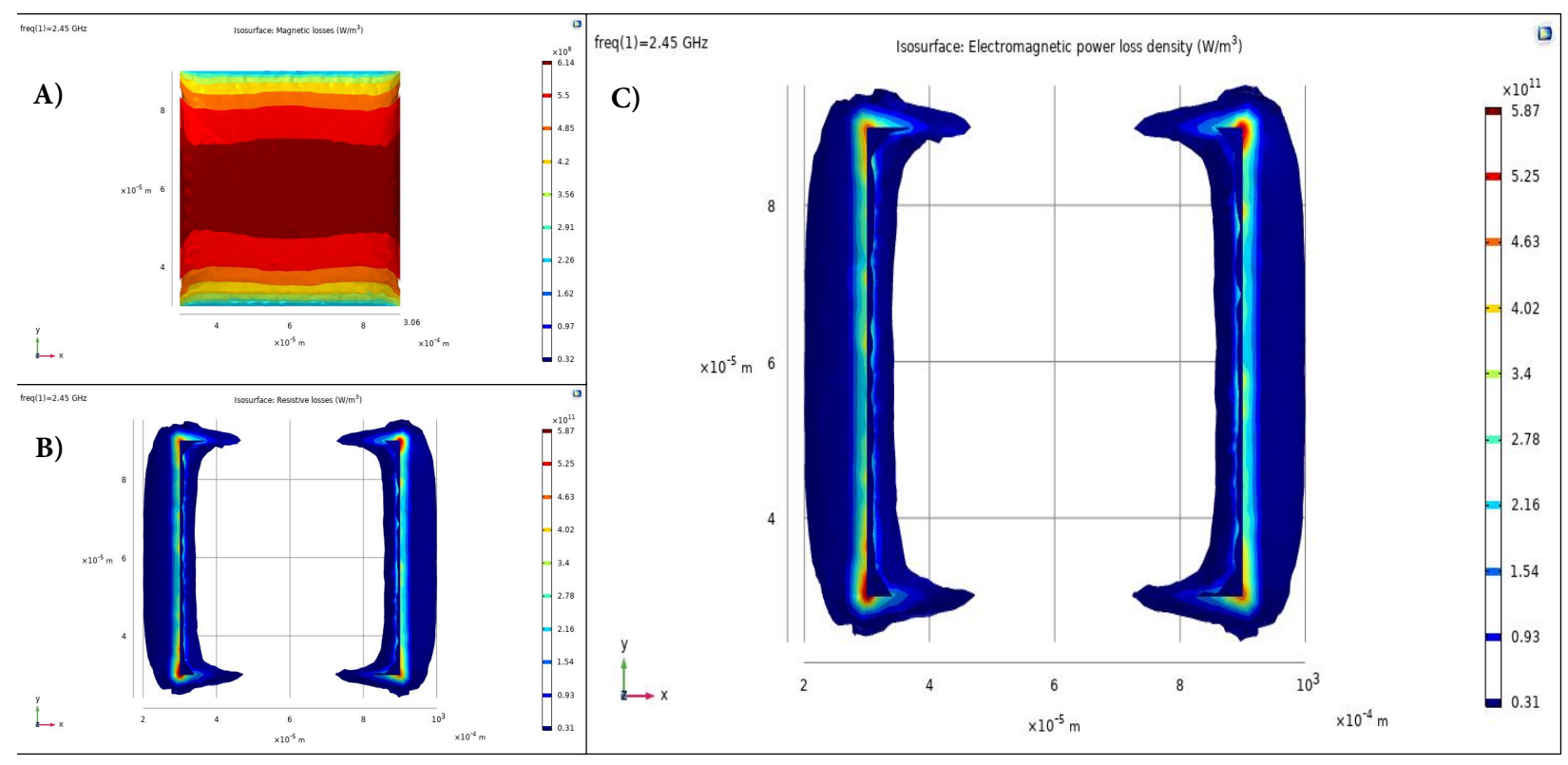

Figure 2.27 Compilation of contour plots illustrating the several magnitude order difference in power loss due to magnetic loss (subfigure A) and electrical (subfigure B) loss. Subfigure $\mathrm{C}$ is the total loss, which is dominated by the electrical field contribution, in accordance with Maxwell's Equations.

shape dependence in the process of microwaving ferromagnetic nanoparticles; concomitant with the benefit of tailoring said shape to enable novel micro-reactor designs using this enhancement of localized dielectric heating. In order to ascertain the most effective shape and generalize how shape affects the heat induced by electromagnetic loss, the geometry of both triangle and square particles had to swept in accordance to their characteristic lengths. The area of an equilateral triangle scales differently than that of a simple quadrilateral, so the parameters and their calculation of area fraction had to be selectively modified to ensure an appropriate comparison of how the size and shape affects the volumetric power generation. Particle morphology preferred the square or rectangular cuboid particles to the triangular prisms, undoubtedly due to the presence of more vertices in the microparticle. The greatest commixture of these variables seems to be the presence of ferromagnetic particles with more vertices (rectangular prisms or higher order prism - perhaps octahedrons like previous analyses on absorption and RL values), with thickness of 5-10 microns, diameter of 60-80 
microns, with a carbon bed at a conductivity around $10 \mathrm{~S} / \mathrm{m}$. The results are catalogued and described by the suite of images from Figure 2.18 to Figure 2.29.
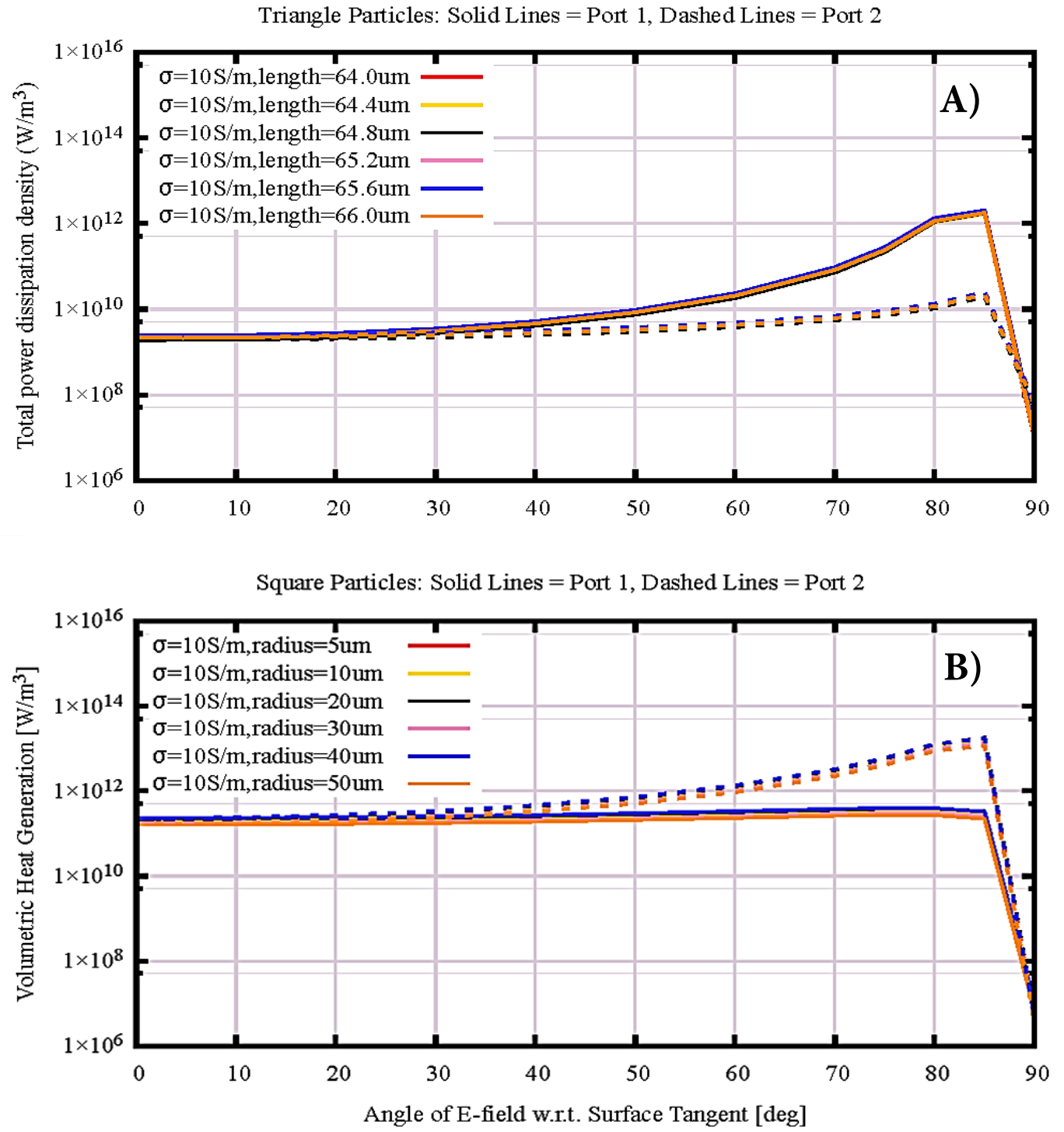

Figure 2.28 Constant area fraction comparison between the triangle (subfigure A) and square-shaped particles (subfigure B). When the characteristic length parameters were swept across in the FEA simulation, the power generation was several fold larger in square-shaped particles than triangular-shaped entities.

Analysis of the thermal gradient at the interface of particle and syn char bed high- 


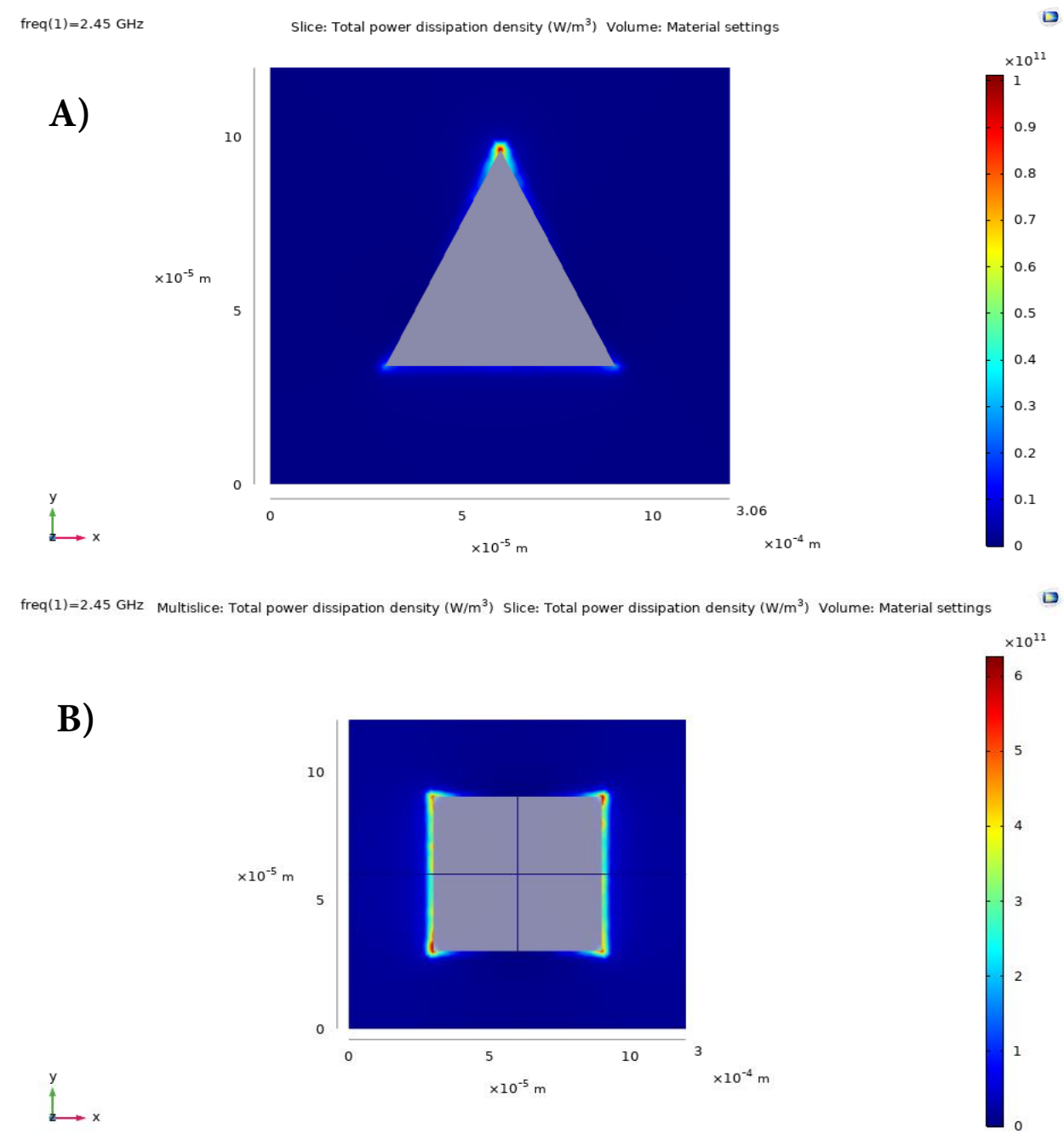

Figure 2.29 Visual depiction of the energy dispersion of the shaped particles. The focusing of electric field energy at the tips of the triangles (subfigure A) can clearly be seen. The dissipation of energy for square-shaped particles (subfigure B) seems to be peripheral all along the edges of the magnetite particle.

lighted how shaped particles can optimize localized heating gradients by inducing large amounts of electrical and magnetic losses at the embedded composite. Starting at an ambient temperature of $293.15 \mathrm{~K}$, the square particle experienced the microwave radiation and immediately responds by heating up the surrounding areas of the square particle. The 2000 Watt microwave energy (non-pulsed) disperses radially form the square particle but heat transfer is greatest on the left and right sides of the particle (looking down on the xy-plane) 
and slowly heats up the reactor.

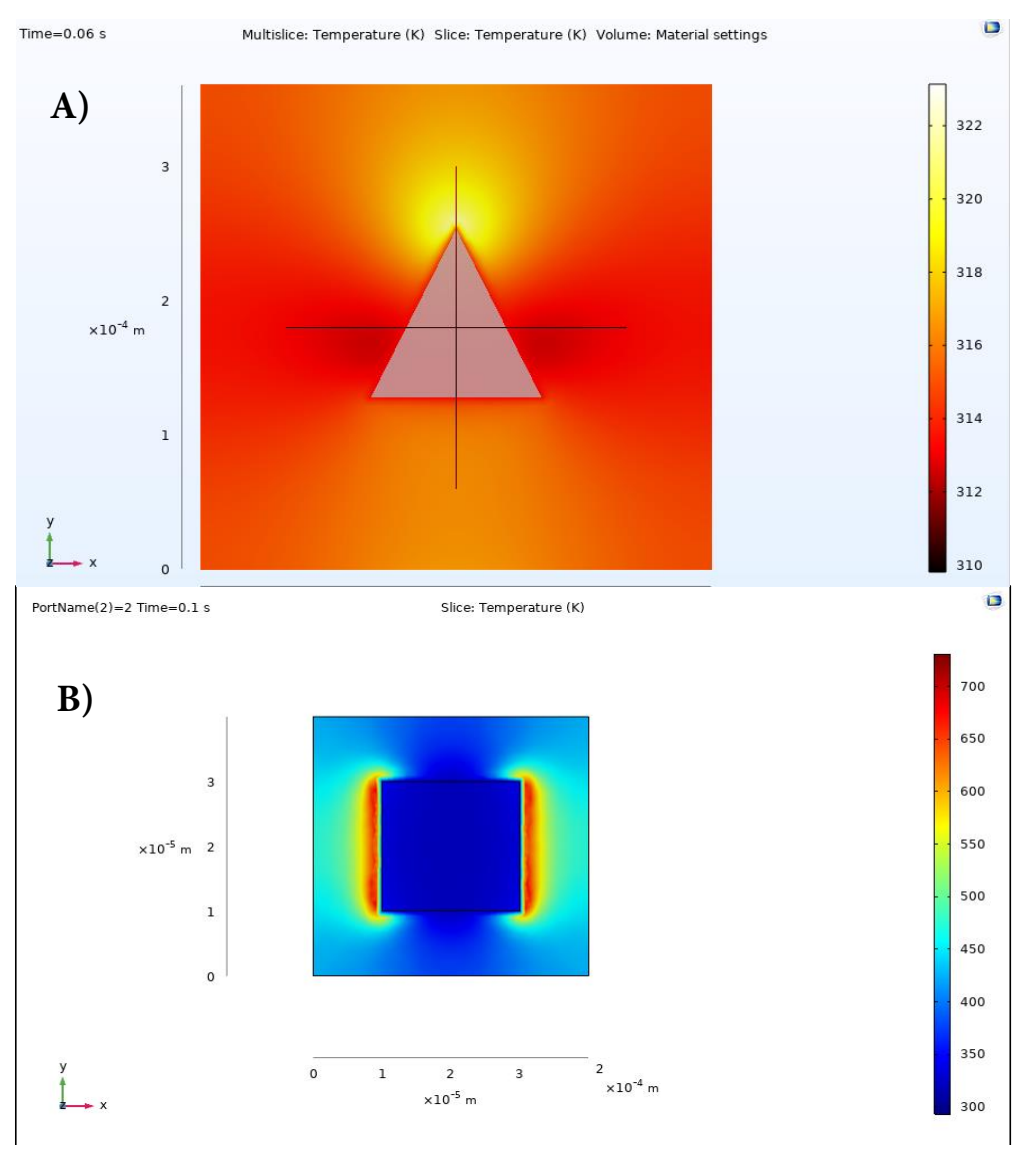

Figure 2.30 Snapshot in time of the heating effect of the magnetite particle when irradiated with $2000 \mathrm{~W}$ microwave energy (non-pulsed) incident 85 degrees to the plane of the particle and bed. Subfigure A (triangle) shows the resultant heating at 0.06 seconds. Subfigure B (square) shows the resultant heating at 0.1 seconds. In both scenarios, the temperature of the local particle surroundings increases dramatically.

Figure 2.30 is an example of this phenomenon in a slice of the xy-plane of the reactor, elucidating the heating action of the particle (square and triangle shapes). Regarding the triangle particle (Figure 2.30A), the local heating effects rapidly converge at 0.06 seconds, albeit more homogeneous in temperature profile than the square particle (Figure 2.30B). The square particle appears to exhibit more directional and robust edge effects in contrast to the 
triangular prisms. After a time-step of 0.1 seconds, the temperature increases dramatically to a temperature of $700 \mathrm{~K}$ or higher localized on the edges, but appears to converge the local temperature profile slower than that of the triangle-shaped magnetite. Knowing this, further modes of research can extend into higher time scales and perhaps the study of many-body interactions (micro-arrays), as the potential applications of these micro-reactors and microarrays are in its infancy. There appears to be "cold" spots where the heating happens at a more constrained pace due to interaction of the conductive syn char bed and ferromagnetic particle and selectively heats where the magnetic flux strength is greatest, i.e. these "cold" spots form where the magnetic field reconnects with the square particle. This could be an interesting analogue to the creation of sun spots on a stellar body where the magnetic field lines enter the domain of the stellar mass, where temperatures are significantly lower in contrast to the rest of the domain; therefore linking the possibility of localized plasma forming at the interface of particle and bed. 


\section{CHAPTER 3}

\section{CONCLUSION}

The characterization and analysis of Powder River Basin coal char have yielded a considerable volume of information regarding the electrochemical properties and dielectric response of a high-rank, sub-bituminous coal sample. Moreover, the development of a coal char simulate provides a fundamental and controlled means of understanding microwave catalytic reaction and the eventual development of micro-reactors with advantageous processing windows. This research aimed at using advanced simulations to guide the experimental development of the PRB coal char simulant. CT image analysis informed a discrete element model that constructed the sample in a similar method as would be done experimentally. This was followed by COMSOL Multiphysics finite element solution that looked at hundreds of trails to determined equivalent dielectric and electrically conductive properties. Using this method the effective dielectric properties of the synthetic coal char material were in good agreement with the actual coal char. It is pertinent to reiterate that this study adequately matched the real electrical dielectric response of Powder River Basin coal char; the elemental composition of PRB coal char; the density of the PRB coal char; the volume fraction of PRB coal char; the interaction of microwave irradiation on PRB coal char; and the electrical conductivity of pyrolzyed PRB. Another crucial finding is the strong evidence that the presence of a shaped, ferromagnetic nanoparticle enables the enhancement of local fields and potentially augment catalytic efficiency. Further analysis on the constituent organic compounds that permeate the coal vessel, and their resultant contribution to constitutive properties detailed in this work is in development. Following first principles, this would be the next step in truly modelling the transformation of Powder River Basin coal (and generic carbonaceous material in general) and elucidating the electromagnetic proper- 
ties seldom analyzed before the introduction of this body of work. Another possible study pathway is modifying the thermal physic simulation with pulsed microwave energy, akin to what is actually used in conventional microwave reactors such as the ones utilized at NETL. In this study, we have only studied the immediate effect of a packet of microwave energy at maximum power of the reactor, but it would behoove future research into seeing how the pulsated beams of energy can interact with the already-present "zoo" of electromagnetic fields near the particle-substrate interface. It is our aim to reinvest and reconstitute carbon-rich materials for novel, chemical reaction engineering, in order to facilitate higher conversion of petroleum products at lesser cost to industry and research, enabling better environmental and economical practices worldwide. 


\section{BIBLIOGRAPHY}

[1] Huanqin Zhao, Yan Cheng, Wei Liu, Zhihong Yang, Baoshan Zhang, Guangbin Ji, and Youwei Du. The flaky porous fe3o4 with tunable dimensions for enhanced microwave absorption performance in x and c bands. Nanotechnology, 29(29):295603, 2018.

[2] Huanpeng Liu, Taiping Chen, and Laixi Fang. Evolution of char structure during nonisothermal low temperature pyrolysis of zhundong coal by microwave heating: A comparative study with conventional heating. Journal of the Energy Institute, 93(3):1195$1206,2020$.

[3] Masahiro Hotta, Miyuki Hayashi, Michael Thomas Lanagan, Dinesh Kumar Agrawal, and Kazuhiro Nagata. Complex permittivity of graphite, carbon black and coal powders in the ranges of $\mathrm{x}$-band frequencies ( 8.2 to $12.4 \mathrm{ghz}$ ) and between 1 and $10 \mathrm{ghz}$. ISIJ international, 51(11):1766-1772, 2011.

[4] Shuqin Liu, Yanjun Zhang, Kaiyong Tuo, Liping Wang, and Gang Chen. Structure, electrical conductivity, and dielectric properties of semi-coke derived from microwavepyrolyzed low-rank coal. Fuel Processing Technology, 178:139-147, 2018.

[5] He Li, Shiliang Shi, Baiquan Lin, Jiexin Lu, Yi Lu, Qing Ye, Zheng Wang, Yidu Hong, and Xiangnan Zhu. A fully coupled electromagnetic, heat transfer and multiphase porous media model for microwave heating of coal. Fuel Processing Technology, 189:4961, 2019.

[6] Robert Meyers. Coal structure. Elsevier, 2012.

[7] Zhihong Qin. New advances in coal structure model. International Journal of Mining Science and Technology, 28(4):541-559, 2018.

[8] Anna Marzec. Towards an understanding of the coal structure: a review. Fuel Processing Technology, 77:25-32, 2002.

[9] Daryl L. Logan. A first course in the finite element method cengage learning, 2011.

[10] Victor Abdelsayed, Dushyant Shekhawat, Mark W. Smith, Dirk Link, and Albert E. Stiegman. Microwave-assisted pyrolysis of mississippi coal: A comparative study with conventional pyrolysis. Fuel, 217:656-667, 2018.

[11] Xinfu He, Lijun Jin, Ding Wang, Yunpeng Zhao, Shengwei Zhu, and Haoquan Hu. Integrated process of coal pyrolysis with co2 reforming of methane by dielectric barrier discharge plasma. Energy \& fuels, 25(9):4036-4042, 2011.

[12] James Baker-Jarvis, Eric J. Vanzura, and William A. Kissick. Improved technique for determining complex permittivity with the transmission/reflection method. IEEE Transactions on microwave theory and techniques, 38(8):1096-1103, 1990.

[13] Wyoming State Geological Survey. Wyoming's coal quality. https://www.wsgs .wyo. gov/energy/coal-geology . aspx, (Last Accessed on 11-15-2021). 
[14] Julia A. Jackson and R.L. Bates. Glossary of geology: Alexandria. Virginia, American Geological Institute, page 769, 1997.

[15] Tiberiu Popa, Maohong Fan, Morris D Argyle, Rachid B Slimane, David A Bell, and Brian F Towler. Catalytic gasification of a powder river basin coal. Fuel, 103:161-170, 2013.

[16] Jürgen Hofmann, Alexander Flisch, and Andreas Obrist. Adaptive ct scanning - mesh based optimisation methods for industrial x-ray computed tomography applications. NDT $\mathcal{E}$ e International, 37(4):271-278, 2004.

[17] 3d micro-tomography - a powerful engineering tool. https://www . coherent.com/lasers/machine-vision?gclid=EAIaIQobChMI1K_bocjJ9AIVh6_ IChOndQV7EAAYASAAEgJZCvD_BwE), Last accessed on 12-4-21.

[18] Gabor T. Herman. Fundamentals of computerized tomography: image reconstruction from projections. Springer Science \& Business Media, 2009.

[19] SERC. X-ray computed tomography (ct). http://www.serc.carleton.edu/msu_ nanotech/methods/CT.html, Last accessed on 07-11-21.

[20] Peter A. Cundall and Otto D.L. Strack. A discrete numerical model for granular assemblies. geotechnique, 29(1):47-65, 1979.

[21] Olek C. Zienkiewicz, Robert L. Taylor, and Jian Z. Zhu. The finite element method: its basis and fundamentals. Elsevier, 2005.

[22] Grenoble University. About, 2009. http://yade-dem.org/wiki/Yade, Last accessed on $05-25-21$.

[23] Ji Xu, Huabiao Qi, Xiaojian Fang, Liqiang Lu, Wei Ge, Xiaowei Wang, Ming Xu, Feiguo Chen, Xianfeng He, and Jinghai Li. Quasi-real-time simulation of rotating drum using discrete element method with parallel gpu computing. Particuology, 9(4):446-450, 2011.

[24] Junuthula Narasimha Reddy. Introduction to the finite element method. McGraw-Hill Education, 2019.

[25] William E. Lorensen and Harvey E. Cline. Marching cubes: A high resolution 3d surface construction algorithm. ACM siggraph computer graphics, 21(4):163-169, 1987.

[26] George Stefanou and Manolis Papadrakakis. Stochastic finite element analysis of shells with combined random material and geometric properties. Computer Methods in Applied Mechanics and Engineering, 193(1-2):139-160, 2004.

[27] COMSOL AB. Comsol multiphysics, 2020. Stockholm, Sweden, http://www.comsol. com, Last accessed on 05-25-21.

[28] Henk G. Merkus. Particle size measurements: fundamentals, practice, quality, volume 17. Springer Science \& Business Media, 2009. 
[29] William B. Weir. Automatic measurement of complex dielectric constant and permeability at microwave frequencies. Proceedings of the IEEE, 62(1):33-36, 1974.

[30] Paul Galvin. Investigation of magnitude and phase errors in waveguide samples for the Nicolson-Ross-Weir permittivity technique. PhD thesis, University of New Hampshire, 2016.

[31] Roy Long. Netl trs, 2016. EDX,http://wwwedx.netl.doe.gov/group/netl-trs., Last accessed on 05-26-21.

[32] Suhui Li and Kevin J. Whitty. Physical phenomena of char-slag transition in pulverized coal gasification. Fuel processing technology, 95:127-136, 2012.

[33] S. Singh, V.B. Neculaes, V. Lissianski, G. Rizeq, S.B. Bulumulla, R. Subia, and J. Manke. Microwave assisted coal conversion. Fuel, 140:495-501, 2015.

[34] Robert Tempke, Liam Thomas, Christina Wildfire, Dushyant Shekhawat, and Terence Musho. Machine learning approach to transform scattering parameters to complex permittivities. Journal of Microwave Power and Electromagnetic Energy, pages 1-16, 2021.

[35] Liang Xu, Haiyu Liu, Yan Jin, Baoguo Fan, Xiaolei Qiao, and Bo Jing. Structural order and dielectric properties of coal chars. Fuel, 137:164-171, 2014.

[36] Alexandre Natã Vicente, Gustavo Maciulis Dip, and Cynthia Junqueira. The step by step development of nrw method. In 2011 SBMO/IEEE MTT-S International Microwave and Optoelectronics Conference (IMOC 2011), pages 738-742. IEEE, 2011.

[37] Wenjun Yin, Duo Dong, Yongsheng Zhang, Tao Wang, Jiawei Wang, Carlos E Romero, and Wei-ping Pan. Effect of annealing temperature on the continuity and conductivity of coal-based carbon films prepared by ball milling. Applied Surface Science, 510:145411, 2020 .

[38] Ruey-Bin Yang, P. Madhusudhana Reddy, Chi-Jung Chang, Po-An Chen, Jem-Kun Chen, and Chung-Chieh Chang. Synthesis and characterization of fe3o4/polypyrrole/carbon nanotube composites with tunable microwave absorption properties: role of carbon nanotube and polypyrrole content. Chemical Engineering Journal, 285:497-507, 2016.

[39] R.K. Walser, W. Win, and P.M. Valanju. Shape-optimized ferromagnetic particles with maximum theoretical microwave susceptibility. IEEE transactions on magnetics, 34(4):1390-1392, 1998.

[40] Antonino Rizzuti, Michele Dassisti, Piero Mastrorilli, Maria C. Sportelli, Nicola Cioffi, Rosaria A. Picca, Elisabetta Agostinelli, Gaspare Varvaro, and Rocco Caliandro. Shapecontrol by microwave-assisted hydrothermal method for the synthesis of magnetite nanoparticles using organic additives. Journal of Nanoparticle Research, 17(10):1-16, 2015 . 
[41] H.R. Gregory, D.C. Rhys Jones, and J.W. Phillips. Compaction of briquettes. Nature, 184(4680):120-121, 1959.

[42] N.A. Abaimov, E.B. Butakov, A.P. Burdukov, and A.F. Ryzhkov. Investigation of steam-air-blown two-stage entrained-flow gasification of mechanoactivated coal. In AIP Conference Proceedings, volume 2015, CID(020001). AIP Publishing LLC, 2018.

[43] Quanlin Hou, Yuzhen Han, Jin Wang, Yijing Dong, and Jienan Pan. The impacts of stress on the chemical structure of coals: a mini-review based on the recent development of mechanochemistry. Science Bulletin, 62(13):965-970, 2017.

[44] John Henry Poynting. On the transfer of energy in the electromagnetic field. Philosophical Transactions of the Royal Society of London, (175):343-361, 1884. 


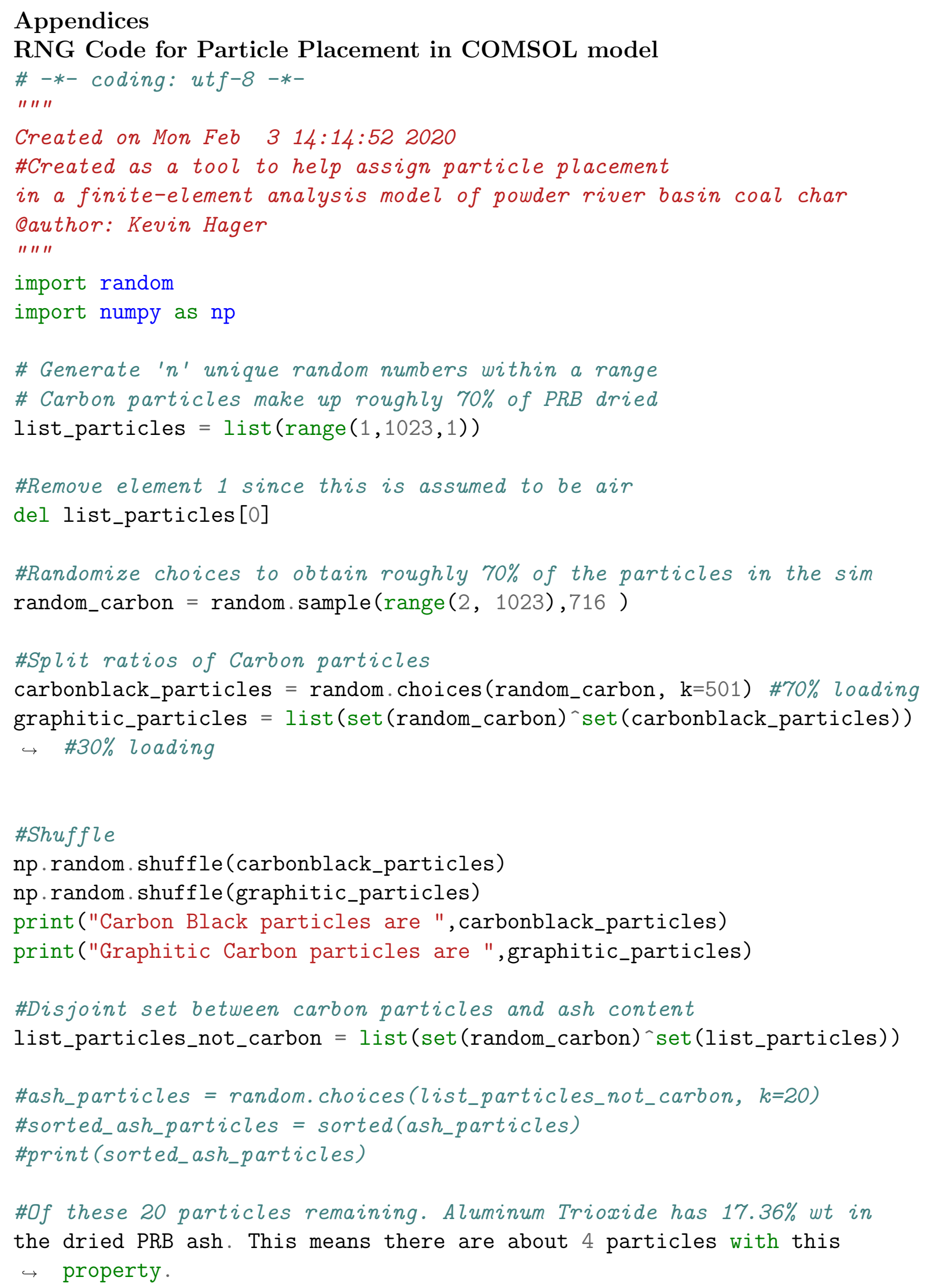


aluminum_oxide_particles = random.choices(list_particles_not_carbon, $\mathrm{k}=53$ ) print("Aluminum Oxide particles are ",aluminum_oxide_particles)

\#There are 16 particles remaining, 37.5\% of ash content in dried PRB is quartz. We can enable 8 particles to have quartz-like properties. remaining_particles $=$ $\hookrightarrow$ list(set(list_particles_not_carbon)^set(aluminum_oxide_particles)) sorted_remaining_particles = sorted (remaining_particles) quartz_particles = random.choices (sorted_remaining_particles, $\mathrm{k}=115$ ) print("Quartz particles are ", quartz_particles)

\#There are roughly $15.3 \%$ of CaO particles in ash content. remaining_particles = $\hookrightarrow$ list(set(sorted_remaining_particles)`set(quartz_particles)) calcium_oxide_particles = random.choices(remaining_particles, k=47) print("Calcium Oxide particles are ",calcium_oxide_particles)

\#There are 198 particles remaining, 3.88\% is iron oxide new_remaining_particles = $\hookrightarrow \quad$ list(set(remaining_particles)^set (calcium_oxide_particles)) iron_oxide_particles $=$ random. choices (new_remaining_particles, $k=12$ ) print("Iron Oxide particles are ",iron_oxide_particles)

\#Sodium Oxide wt\% is 5.95\% in Dried PRB new2_remaining_particles = $\hookrightarrow \quad$ list (set (new_remaining_particles)^set(iron_oxide_particles)) np.random.shuffle (new2_remaining_particles) sodium_oxide_particles =random. choices (new2_remaining_particles, $\mathrm{k}=18$ ) print("Sodium Oxide particles are ",sodium_oxide_particles)

\#Magnesium Oxide wt \% is $4.68 \%$

new3_remaining_particles = $\hookrightarrow \quad$ list(set (new2_remaining_particles)`set(sodium_oxide_particles)) np.random.shuffle(new3_remaining_particles) magnesium_oxide_particles =random. choices (new3_remaining_particles, $\mathrm{k}=14$ ) print("Magnesium Oxide particles are ",magnesium_oxide_particles)

new4_remaining_particles $\hookrightarrow \quad=l i s t($ set(new3_remaining_particles)`set(magnesium_oxide_particles)) $\mathrm{np}$.random.shuffle(new4_remaining_particles) print("Remaining particles in the simulation are ",new4_remaining_particles) 


\section{NRW Polynomial Interpolation Code}

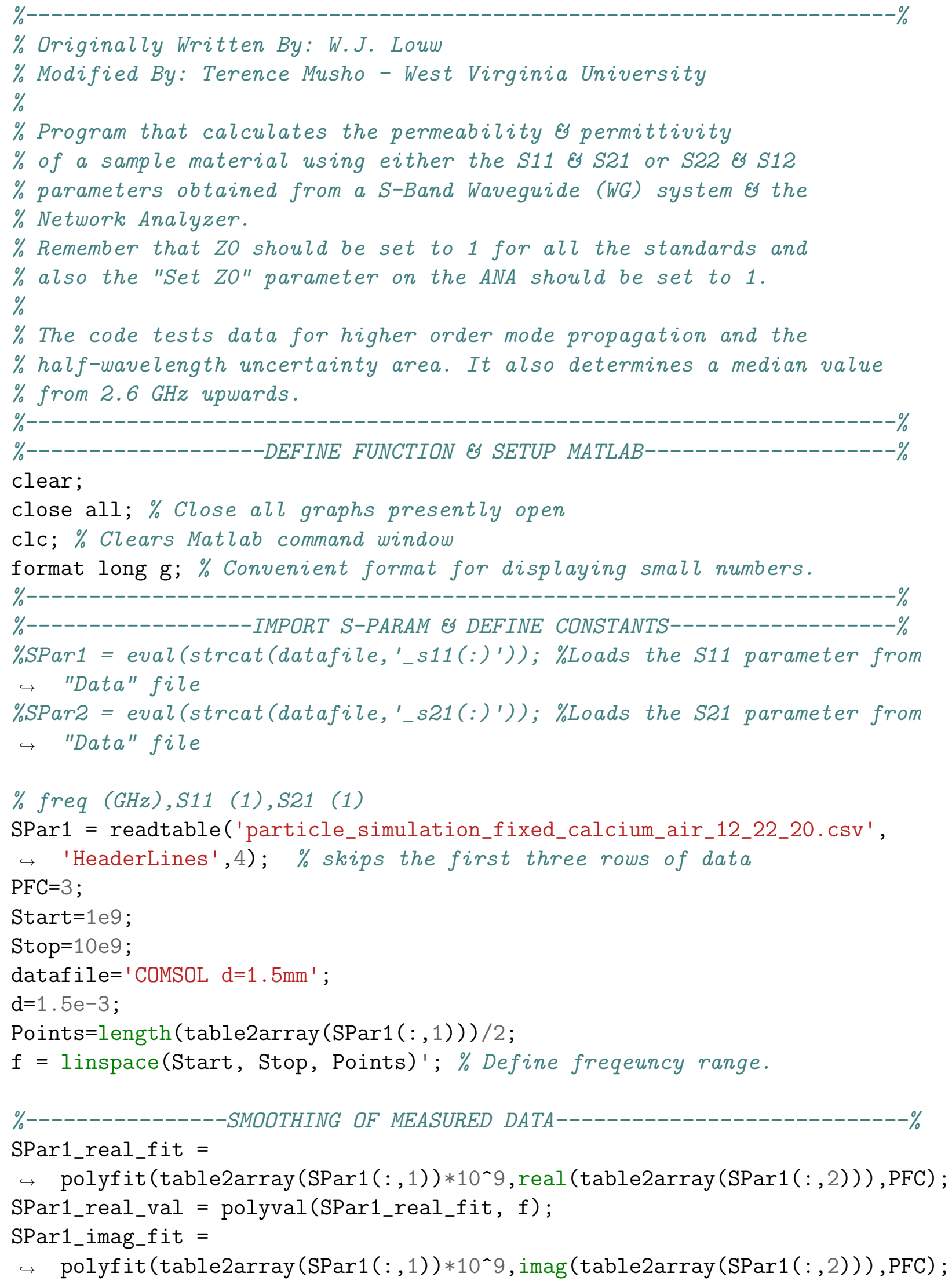




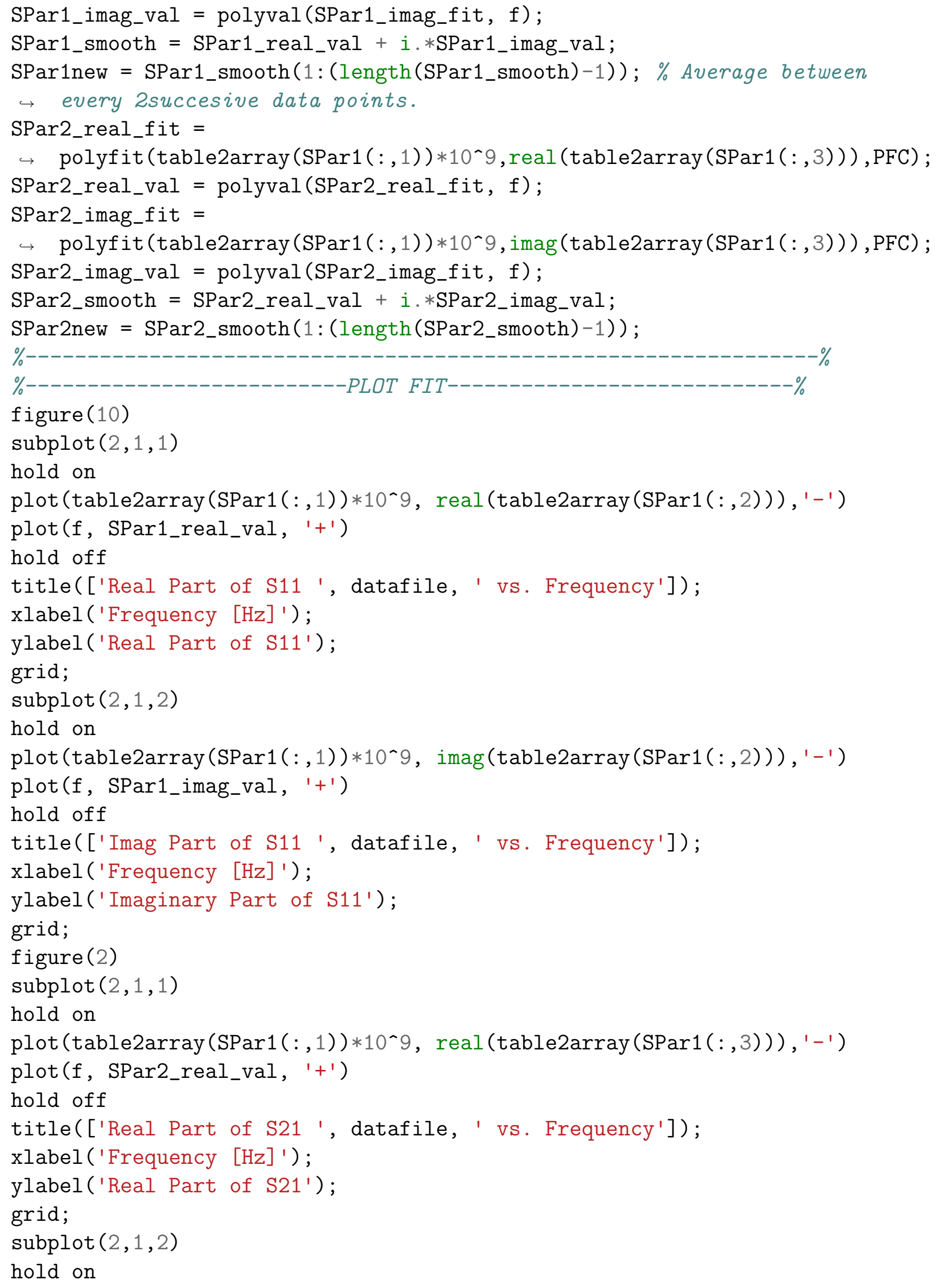


plot (table2array (SPar1 $(:, 1)) * 10^{-9}$, $\left.\operatorname{imag}(\operatorname{table2\operatorname {array}}(\operatorname{SPar} 1(:, 3))),^{\prime} \mathbf{-}^{\prime}\right)$ plot (f, SPar2_imag_val, '+')

hold off title(['Phase of S21 ', datafile, ' vs. Frequency']); xlabel ('Frequency $[\mathrm{Hz}]$ '); ylabel ('Imaginary Part of S21'); grid;

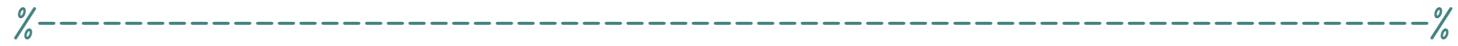




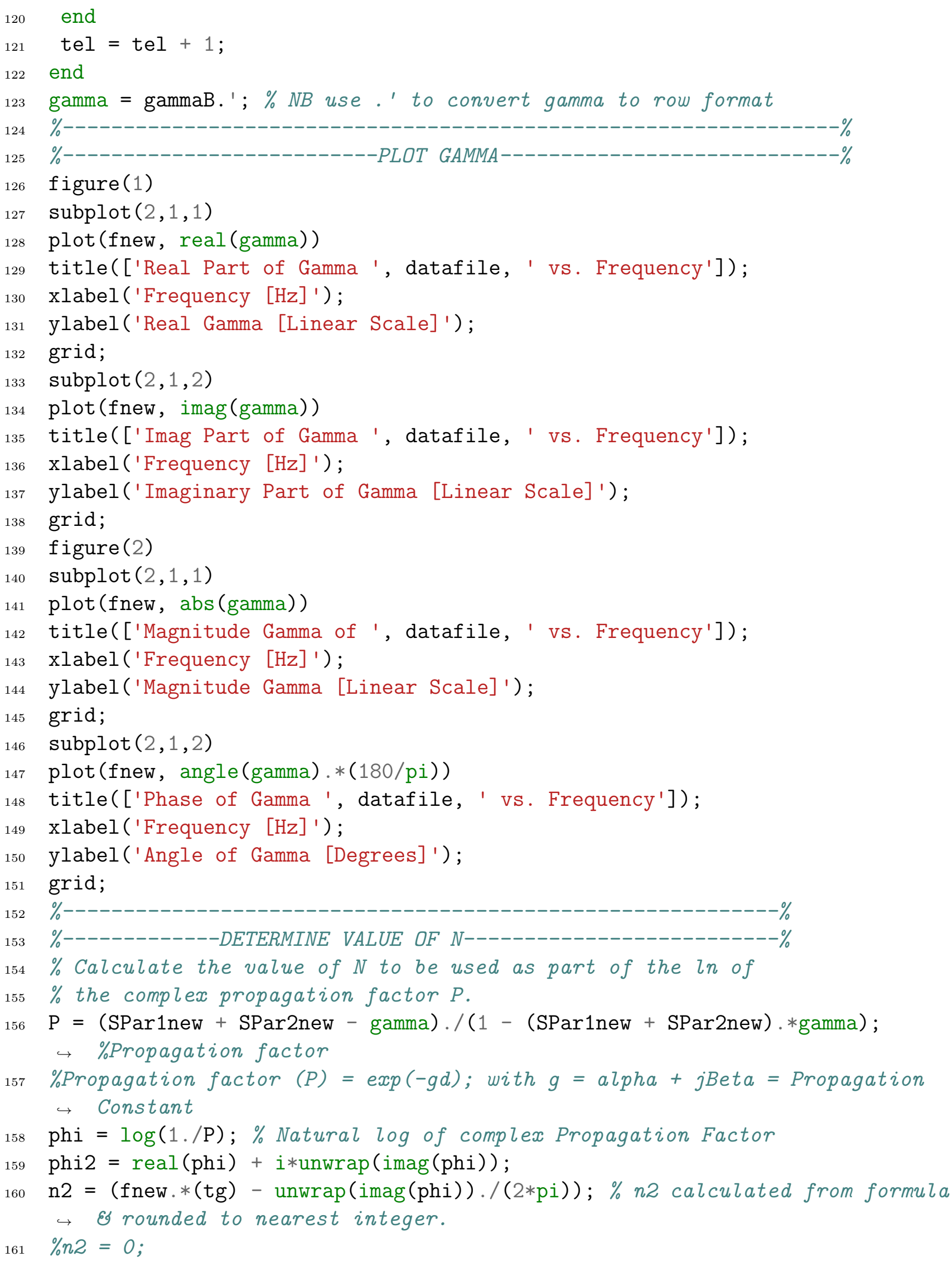




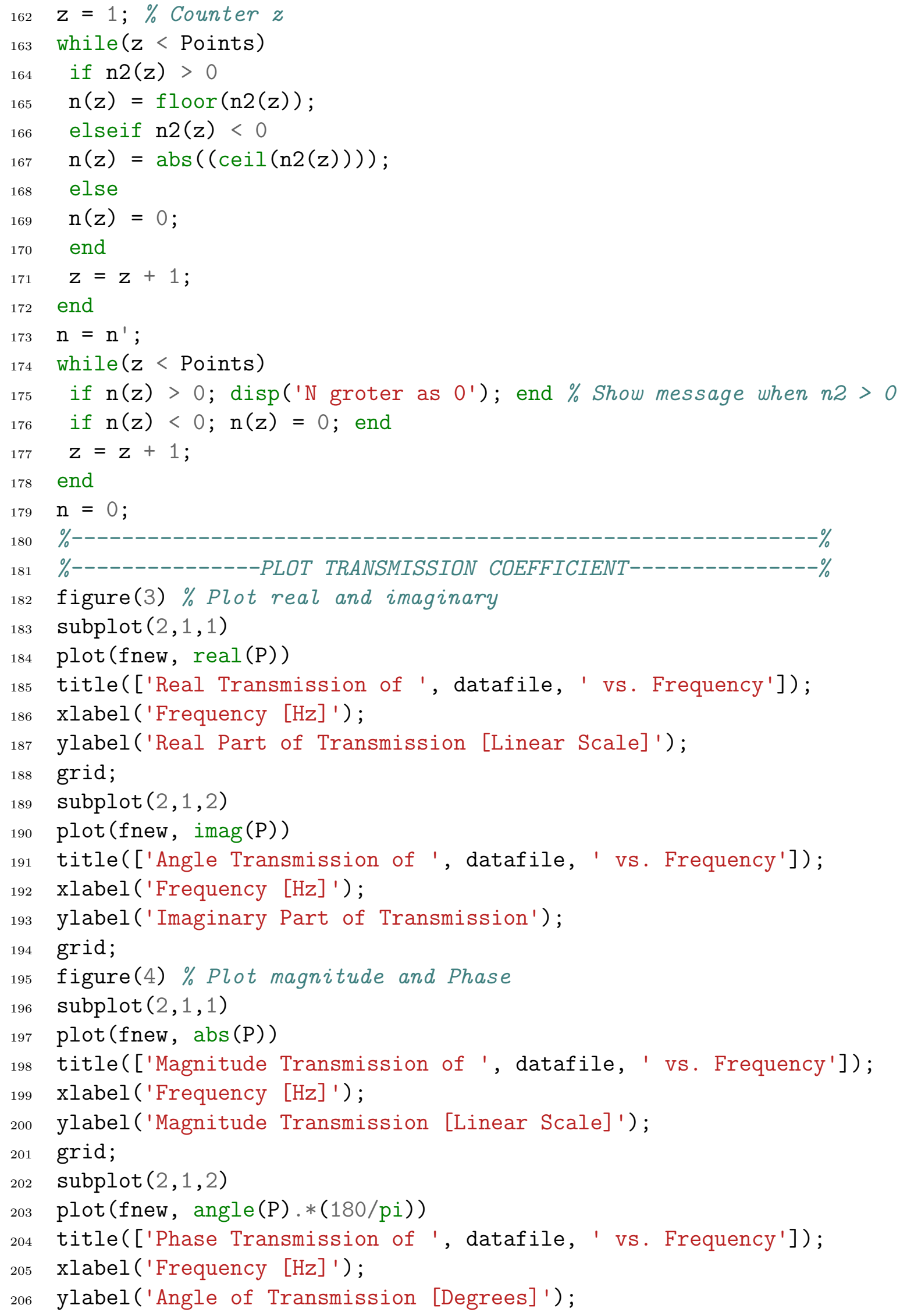




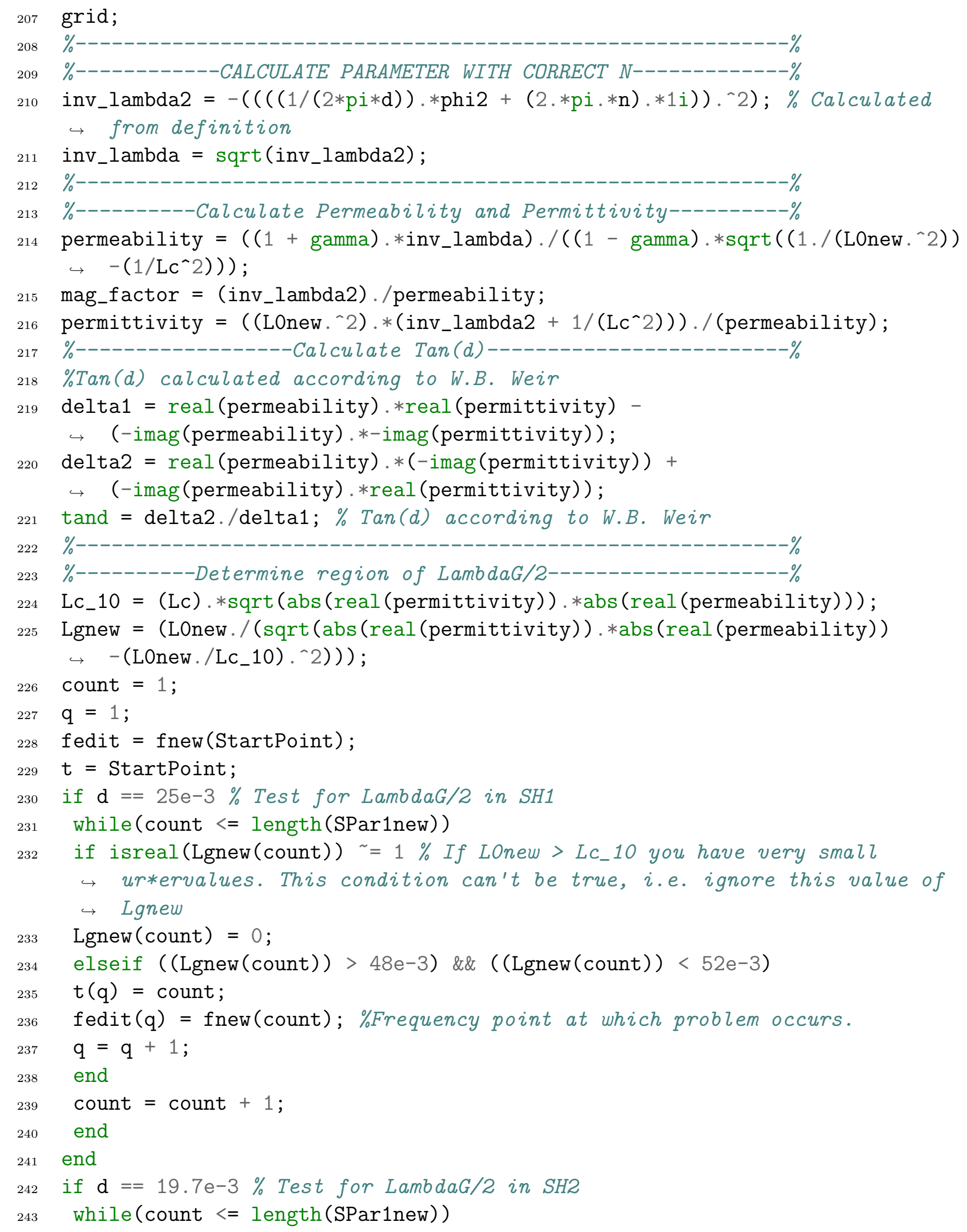




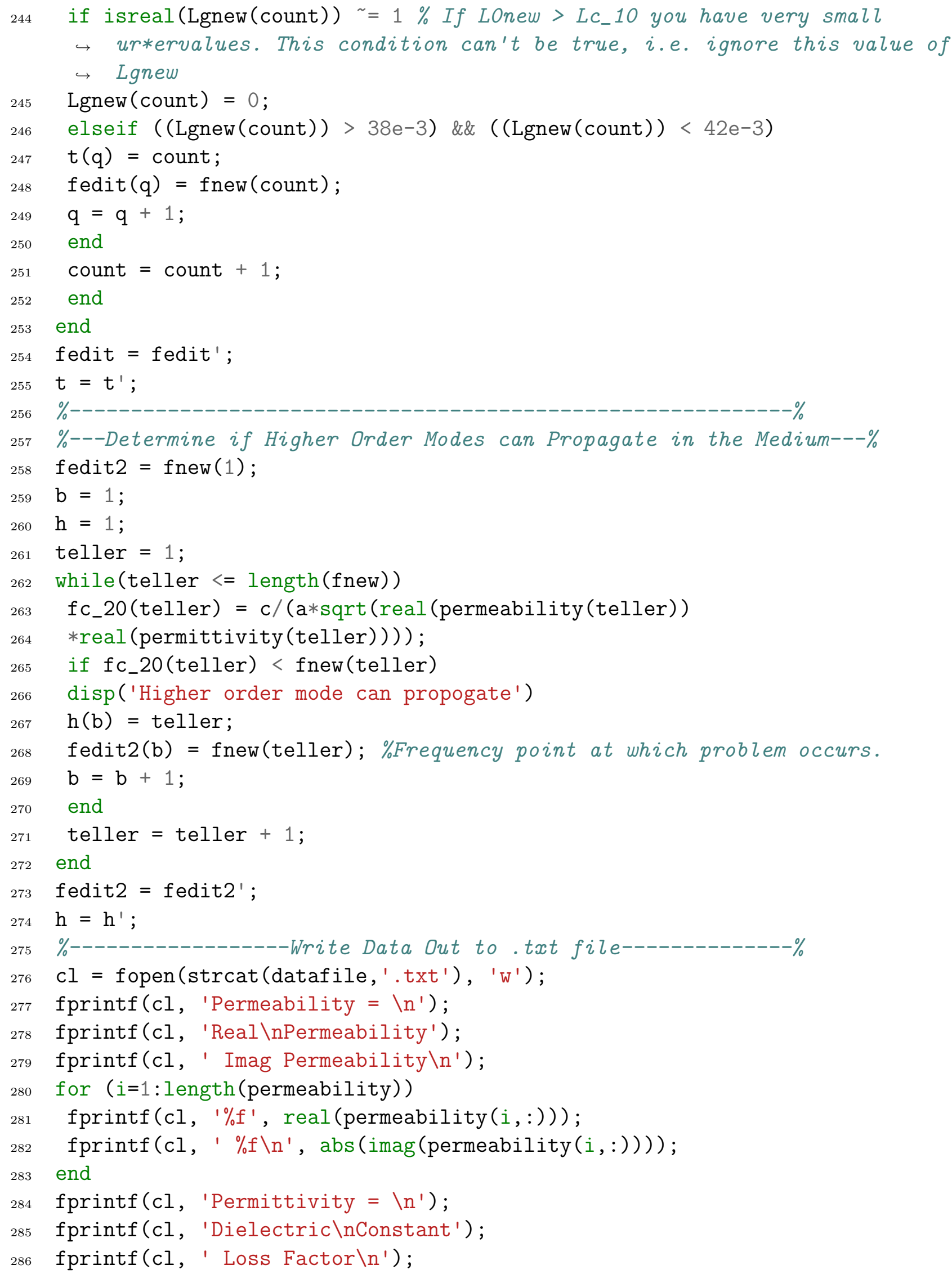




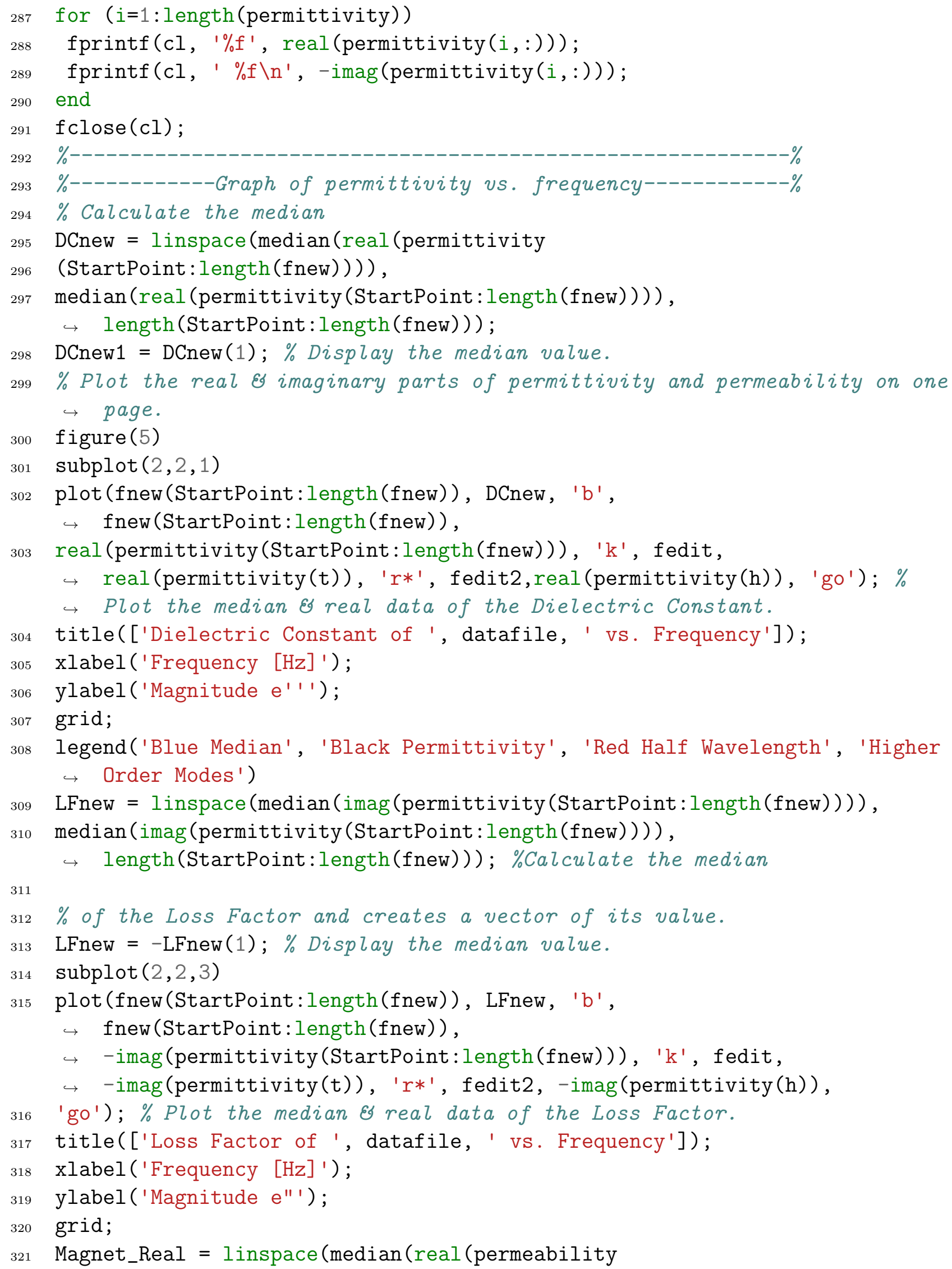


(StartPoint: length(fnew))) ), median (real (permeability

(StartPoint:length(fnew)))), length(StartPoint:length(fnew))); \%Calculate $\rightarrow$ the median

Magnet_Real $=$ Magnet_Real (1); \% Display the median value. subplot $(2,2,2)$

plot (fnew (StartPoint: length(fnew)),

$\hookrightarrow \quad$ real (permeability (StartPoint:length(fnew))),

$\hookrightarrow$ 'k',fnew(StartPoint:length(fnew)), Magnet_Real, '-b', fedit2,

$\hookrightarrow$ real(permeability(h)), 'go', fedit, real(permeability(t)), 'r*');\% Plots

$\rightarrow$ the real part

title(['Real part of permeability of ', datafile, 'vs. Frequency']); xlabel ('Frequency $[\mathrm{Hz}]$ ');

ylabel ('Magnitude u''');

grid;

Magnet_Imag $=$ linspace $($ median $($ imag $($ permeability

(StartPoint:length(fnew)))), median (imag (permeability (StartPoint: length(fnew)))),

$\rightarrow$ length(StartPoint:length(fnew)));

\% Calculate the median

Magnet_Imag $=-$ Magnet_Imag(1); \% Display the median value. subplot $(2,2,4)$

plot(fnew(StartPoint:length(fnew)), Magnet_Imag, 'b',

$\rightarrow$ fnew(StartPoint:length(fnew)),

$\hookrightarrow$-imag(permeability(StartPoint:length(fnew))), 'k', fedit,

$\rightarrow \quad-i m a g($ permeability(t)), 'r*', fedit2,-imag(permeability(h)), 'go'); \%

$\rightarrow$ Plots the imaginary part.

title(['Imaginary part of permeability of ', datafile, ' vs. Frequency']); xlabel ('Frequency $[\mathrm{Hz}]$ ');

ylabel ('Magnitude u"');

grid;

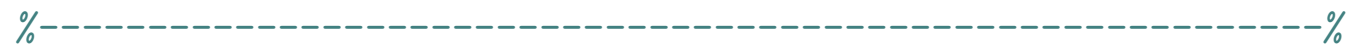

$\%-----------G r a p h$ of $\tan (\operatorname{delta})$ vs. frequency---------------\%

figure (6)

plot (fnew, tand);

title(['Tan(delta) of ', datafile, ' vs. Frequency']);

xlabel ('Frequency $[\mathrm{Hz}]$ ');

ylabel ('Magnitude tan(delta)');

grid;

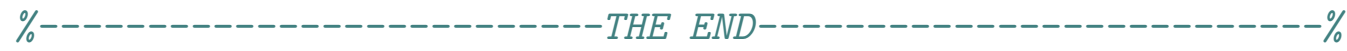

